\title{
Studying Simulations with Distributed Cognition
}

by

\section{Jonas Rybing}

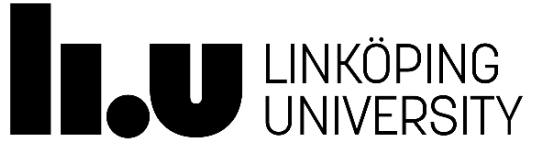
Department of Computer and Information Science Linköping University
SE-581 83 Linköping, Sweden


Cover photo copyright (C) Erik Prytz and used with permission. All photos in the thesis are used with permission and all publications have been reprinted with permission of the respective copyright owners.

The typesetting is Garamond: http://www.georgduffner.at/ebgaramond/

\section{Copyright (C) 2018 Jonas Rybing}

ISBN 978-91-7685-348-1

ISSN 0345-7524

Printed by LiU-Tryck 2018

URL: http://urn kb.se/resolve?urn=urn nbn:se:liu:diva-145307 


\section{Abstract}

Simulations are frequently used techniques for training, performance assessment, and prediction of future outcomes. In this thesis, the term "human-centered simulation" is used to refer to any simulation in which humans and human cognition are integral to the simulation's function and purpose (e.g., simulation-based training). A general problem for human-centered simulations is to capture the cognitive processes and activities of the target situation (i.e., the real world task) and recreate them accurately in the simulation. The prevalent view within the simulation research community is that cognition is internal, decontextualized computational processes of individuals. However, contemporary theories of cognition emphasize the importance of the external environment, use of tools, as well as social and cultural factors in cognitive practice. Consequently, there is a need for research on how such contemporary perspectives can be used to describe human-centered simulations, re-interpret theoretical constructs of such simulations, and direct how simulations should be modeled, designed, and evaluated.

This thesis adopts distributed cognition as a framework for studying human-centered simulations. Training and assessment of emergency medical management in a Swedish context using the Emergo Train System (ETS) simulator was adopted as a case study. ETS simulations were studied and analyzed using the distributed cognition for teamwork (DiCoT) methodology with the goal of understanding, evaluating, and testing the validity of the ETS simulator. Moreover, to explore distributed cognition as a basis for simulator design, a digital re-design of ETS (DigEMERGO) was developed based on the DiCoT analysis. The aim of the DigEMERGo system was to retain core distributed cognitive features of ETS, to increase validity, outcome reliability, and to provide a digital platform for emergency medical studies. DigEmergo was evaluated in three separate studies; first, a usefulness, usability, and facevalidation study that involved subject-matter-experts; second, a comparative validation study using an expert-novice group comparison; and finally, a transfer of training study based on self-efficacy and management performance. Overall, the results showed that DigEMERGo was perceived as a useful, immersive, and promising simulator - with mixed evidence for validity - that demonstrated increased general self-efficacy and management performance following simulation exercises.

This thesis demonstrates that distributed cognition, using DiCoT, is a useful framework for understanding, designing and evaluating simulated environments. In addition, the thesis conceptualizes and re-interprets central constructs of human-centered simulation in terms of distributed cognition. In doing so, the thesis shows how distributed cognitive processes relate to validity, fidelity, functionality, and usefulness of human-centered simulations. This thesis thus provides a new understanding of human-centered simulations that is grounded in distributed cognition theory.

The research has been supported by the Swedish Civil Contingencies Agency (MSB) within the Centre for Advanced Research in Emergency Response (CARER) at Linköping University. 


\section{Preface}

When I started this adventure into academia I thought that I knew what was ahead of me. It turned out that I did not. In particular, I did not anticipate that simulations would become such a large part of my life. As a fresh undergraduate student of cognitive science (Linköping University-style), I did not think much of simulations. In my world, they were mostly lab experiments that could not tell us much about real life or cognition. At their best, they could be impressive pieces of technology or entertaining games. Little did I know. Five years later and now you, dear reader, are holding a full thesis text dedicated to them. What makes simulations so fascinating is that they are parallel realities, or another form of reality, that we seem to attach ourselves to. I have seen, and experienced, how even simple simulations can create such a dramatic sense of immersion and emotional response for those who engage with them. This is where I got hooked.

During the entirety of my graduate studies I have struggled, more or less consciously, with philosophy of science questions: what is science, what is engineering, and where do the two meet? Perhaps such questions are natural to any member of the Department of Computer and Information Science, but I believe that these questions have haunted me more than most. This thesis is my way of answering such questions for myself, to the degree that I can, and I think that my ponderings are evident throughout the text.

There are, of course, many people to be grateful to and to thank for making this thesis a reality. First, I want to thank my supervisors. Without them there simply would have been no thesis. I want to thank my co-supervisor Erik Prytz for our many interesting discussions, his ideas, and constant support. And for always letting me pick his brain when my own had an itch. Without Erik's help, this thesis would have been both a conceptual and a grammatical mess. You have taught me many things - dos and don'ts that are necessary in order to write a clear and compelling academic text. It has been a true pleasure to work with you, and I hope that our paths will cross again in the future.

Next, thank you to my supervisor Magnus Bång for always listening and looking out for the person behind the graduate student. The road to this thesis was not a straight one, and I want to thank you for keeping me on track. You are a visionary, and even though many of our ideas have not yet come to fruition, it has always been inspiring to talk to you and discuss future projects and ideas with you.

I also want to thank my co-supervisors Henrik Eriksson and Sofie Pilemalm for helping me along the way. A special thanks to Henrik, whose keen eye and experience helped me attend to what needed to be done to complete the thesis when many threads were hanging loose. Speaking of loose threads, thank you to Anne Moe for guiding me - and my tired mind - through the jungle of important dates, appointments, formats, and forms that come with the thesis and dissertation process.

The research in this thesis could not have been done without the enthusiastic aid from everybody over at the Centre for Teaching \& Research in Disaster Medicine and Traumatology (KMC). Special thanks go out to Carl-Oscar Jonson, Heléne Nilsson, Johan Hornwall, Jenny Petterson, and Eva Bengtsson 
for your vigorous participation in this research, and in the development of DIGEMERGO in particular. This system would not exist without you, or without our excellent programmers Mattias Lantz Cronqvist and Carl Einarson.

I would also like to thank the CARER research group and especially my fellow graduate-students in the group. Discussing with you was a big help and a much needed safety-net during the start of this adventure.

A big thanks goes out to all of you graduate-fellows at IDA. There have been so many of you over the years that I will not list all of you here for fear of leaving someone out. Our daily fika-breaks and our PhD-pubs were all important departures from reality - or perhaps they were much-needed visits to reality - that really helped me get through it all. Special thanks to Mattias Forsblad and Lisa Malmberg, who wandered $\mathrm{PhD}$-paths parallel to mine. We shared many trips to the fruit room, inspiring chats about everything and nothing, and volleyball games. Special thanks also go out to my friends Christian Smith and Robin Keskisärkkä for the countless, often nerdy, discussions and our yearly fishing trips.

At the end of a long academic day, the best medicine was often to mindlessly run and run some more after a ball. For that I want to thank the football-gang BK69, and especially Sture Hägglund who introduced me to this wonderful gang of players. Speaking of players, I would like to thank the Heroes of Golarion-group for our many imaginary adventures into the unknown and our subsequent encounters with danger. Special shout out to GM Johan Jäger for the many hours of preparation and work you have put in to steer us through the story.

I would like to take the opportunity to thank my family for providing me with the chance and continuous support to take this academic expedition. Perhaps someday it will become clear what I have been doing during all of these years. Finally, I would like, with all my heart, to thank my love Emma, who has endured with me and my extensive thesis work. Without your love and support, I would not have made it across the finish line.

Thank you all big big!

Jonas Rybing

A snowy day at Strånganäs, March 2, 2018 


\section{Thesis Contents}

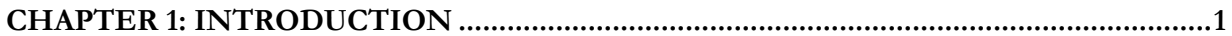

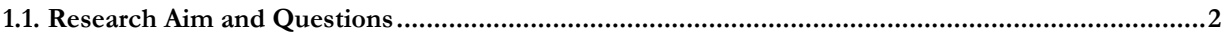

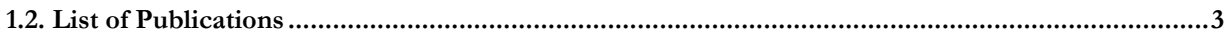

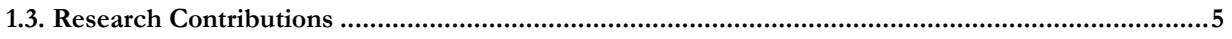

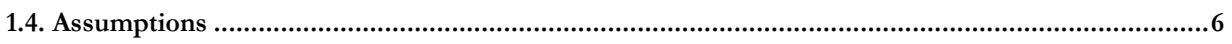

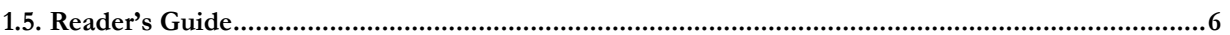

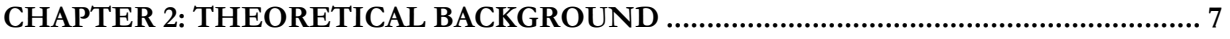

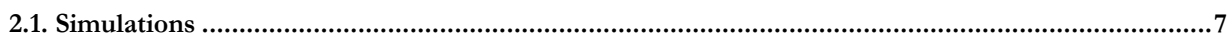

211 A Brief History of Simulation 7

212 The Simulation Discipline 9

213 Simulation Constructs 17

214 Simulation for Training 24

215 The Human in Simulation $\quad 27$

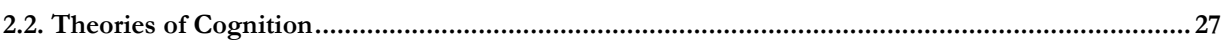

221 What is Cognition? 27

222 Distributed Cognition Perspective 30

CHAPTER 3: EMERGENCY MEDICAL MANAGEMENT DOMAIN …...........................37

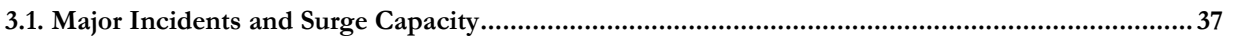

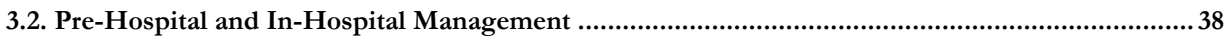

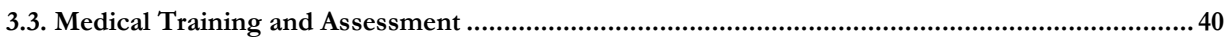

331 Use of Simulations $\quad 40$

332 The Emergo Train System $\quad 40$

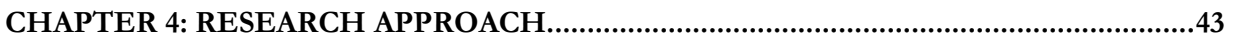

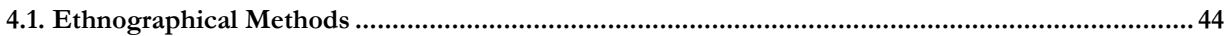

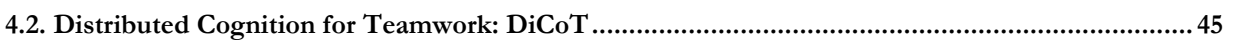

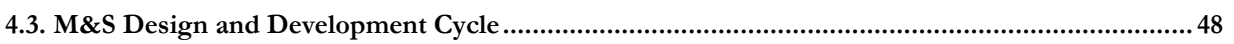

431 Modeling $\quad 48$

432 Construction 49

433 Execution $\quad 50$

434 Analysis $\quad 50$

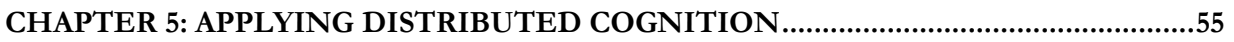

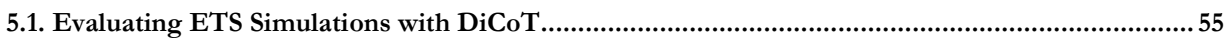

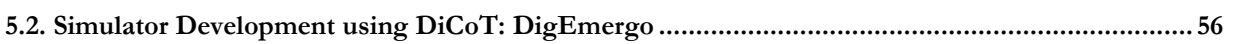

521 Modeling $\quad 57$

522 Construction $\quad 57$

523 Analysis 58

CHAPTER 6: RE-INTERPRETING SIMULATION CONCEPTS ....................................61

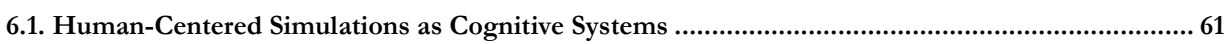


6.2. Interpreting Descriptive Attributes

621 Cognitive Fidelity

622 Resolution and Scale

6.3. Interpreting Validity

631 External Validity

632 Internal Validity

633 Representational Validity

634 Application Validity

CHAPTER 7: DISCUSSION AND CONCLUSION

7.1. Addressing Research Questions

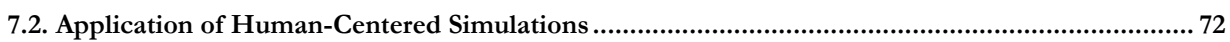

7.3. Contributions as Products of Technological Research and Science .......................................... 74

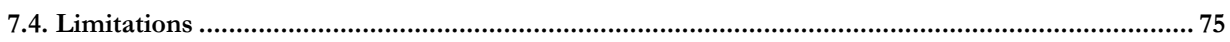

741 The DigEmergo Design Case $\quad 75$

742 Mixed DigEmergo Validation Results $\quad 76$

743 Alternative Research Approaches $\quad 77$

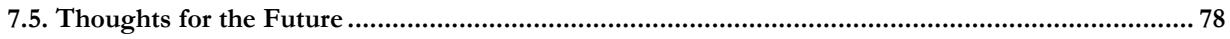

751 Additional Validation Studies of DigEmergo $\quad 78$

752 Development of DiCoT $\quad 78$

753 DigEmergo Development $\quad 79$

$\begin{array}{ll}754 \text { A Philosophical Note } & 79\end{array}$

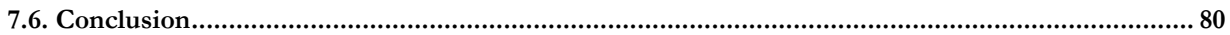

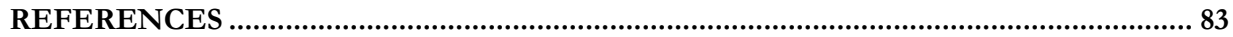

\section{Figures}

Figure 1: Central concepts of simulation $\quad 12$

Figure 2: Simulation concepts enriched with Winsberg's (2015) definition of a simulation 15

Figure 3: Map of the validity concepts and their relations $\quad 22$

Figure 4: Simulation concepts combined with validity concepts and descriptive attributes 24

Figure 5: ETS simulation participant engaged in work at an accident site 41

Figure 6: Two ETS magnetic patient figures $\quad 42$

Figure 7: Published papers and studies $\quad 43$

Figure 8: Major phases of the simulation development cycle 48

Figure 9: Example outtake from a claims analysis in Furniss and Blandford (2006) 49

Figure 10: Evaluation of DigEMERGO $\quad 58$

Figure 11: Examples of different user-types in human-centered simulations $\quad 74$

\section{Tables}

$\begin{array}{ll}\text { Table 1: Examples of suggested definitions } & 14\end{array}$

Table 2: Examples of fidelity types from the literature $r$

Table 3: Concepts related to validity found in the literature $\quad 20$

Table 4: Principles of DiCoT Principles of distributed cognition categorized according to the five models 46 


\section{CHAPTER 1 INTRODUCTION}

The topic of this thesis is the understanding of simulations where human interaction and cognition are central to the functionality and intended results of the simulation. I will refer to such simulations as buman-centered simulations. Setting formal definitions aside for later, a simulation is simply an activity that is intended to recreate some real-world phenomena (e.g., activities, environments, behaviors, or processes) using simplification, selection, and abstraction of the phenomena. This inclusive description illustrates that human-centered simulations, and simulators (the 'machines' used for creating or supporting simulations), can be many different things. In fact, one could argue that we all engage in human-centered simulation on a daily basis. For instance, a chess player simulates abstract military strategy, a video gamer playing a racing game simulates driving, medical students using laparoscopic tools to cut open the skin of an orange are simulating a surgical procedure, and football practice is a form of match-day simulation.

The case used in this thesis to study human-centered simulation is simulation-based training (SBT) environments for training medical personnel in emergency medicine management. There are many different types of SBT systems within the medical domain, from technologically basic to advanced (Maran \& Glavin, 2003). These systems range from tabletop role-play simulations that focus on communication (Chi et al., 2001), to training exercises that use actors or advanced medical mannequins (see e.g. Gillett et al., 2008), to fully virtual or augmented environments that recreate detailed accident or hospital scenarios (Cicero et al., 2015; Knight et al., 2010). Considering the wide range of simulation types that are available, an obvious question comes to mind: which simulation is the best one to use? This is not easy to answer as it raises a set of scientific questions concerning which simplifications, selections, and abstractions would best serve the intended purpose of the simulation. These questions need further theoretical characterization. For example, a simulation should capture the correct things from reality in a correct manner. The degree of success in doing so is broadly referred to as the validity of a simulator (Feinstein \& Cannon, 2001; Pace, 2004; Sokolowski \& Banks, 2010; Winsberg, 2015). However, how do we know what these correct things are and which manner is correct? It is easy to imagine that an exact, or near exact, replica of the real situation would be the best choice and the most valid simulation. The following quotation from Rosenblueth and Wiener (1945) express this idea in an elegant way:

"The best material model of a cat is another, or preferably the same, cat." (p. 320)

However, such hyper-real simulations would be expensive, complicated, time-consuming, impractical, and in many cases, too dangerous or ethically impossible - thereby sacrificing many of the benefits of simulations. Furthermore, it has been shown that the absence of realism can make training simulations more efficient, for instance through task simplification and highlighting of key skills (Alessi, 1988; 
Drews \& Bakdash, 2013). Consequently, SBT researchers argue that the things of importance are not those that make the simulation appear real; rather, it is things that capture the underlying psychological processes of the target tasks (Kozlowski \& DeShon, 2004). A human-centered simulation that is suitable for training could, therefore, be seen as one that engages relevant cognitive processes to generate positive transfer of targeted skills and knowledge to the real situation.

An appropriate theoretical framework for cognition is needed to understand the cognitive processes at work in both reality and in simulation. Typically, simulation research, tends to treat cognition in a traditional manner that views cognition as internal computational processes solely confined within the head. However, such traditional views on cognition have been challenged within contemporary cognitive science and human factors (Hollan, Hutchins, \& Kirsh, 2000; Hutchins, 1995a, 1995b; Norman, 1994; Perry, 2003). Alternative contemporary theories of cognition claim that the outside world both shapes and is part of our cognition, for example through physical tools, spatial layout, social structures, and cultural practices. Such external aspects create a cognitive environment that enables and mediates cognitive activities and capabilities. Distributed cognition is one such framework of, or perspective on, cognition that emphasizes the importance of the cognitive environment and cognition in context. From this perspective, cognitive processes are seen as being distributed across individuals, artifacts, and time within a social and cultural setting (Hutchins, 1995a).

In this thesis, I suggest that the traditional view of cognition is insufficient for explaining central phenomena and theoretical constructs in simulation theory and practice. This is because the traditional view does not account for how the cognitive environment contributes to and shapes cognitive processes during simulations and training exercises. A new approach is needed to further our understanding of how simulators shape the cognitive environment and mediate cognition within simulation. Adopting a contemporary perspective on cognition can affect simulator development and evaluation concerning what simplifications, selections, and abstractions to make. In this thesis, I adopt the distributed cognition framework to re-interpret central concepts in simulation theory and explore how simulated training environments and simulators can be studied, evaluated, and designed. An underlying hypothesis of this work is that distributed cognition can provide a deeper and more comprehensive understanding of human-centered simulations and core simulation concepts than traditional cognitive perspectives.

\subsection{Research Aim and Questions}

The overall aim of this thesis is to adopt and explore the usefulness of distributed cognition as a framework for studying and understanding human-centered simulations and to discuss its implications for simulation theory. A second overall aim is to use the distributed cognition framework to design human-centered simulators. These aims were investigated through the distributed cognition in teamwork (DiCoT) methodology (Furniss \& Blandford, 2006) using SBT of emergency medical management as a case study. In particular, simulations using the Emergo Train System (ETS; Nilsson, Vikström, \& Rüter, 2010) simulator were studied. The case is suitable because emergency medical management consists of several cognitive tasks - such as critical decisions regarding patient prioritization (triage), treatment, and transportation - that involve coordination between individuals, tools, and technology. Furthermore, to investigate the use of distributed cognition for design of human-centered simulations, 
a digital training-simulator prototype (DigEmergo) was developed based on the DiCoT analysis of ETS. This thesis addresses the following research questions:

R.Q.1. How can distributed cognition be used for evaluation and (re)design of buman-centered simulations?

R.Q.2. Is the DiCoT methodology a useful approach for studying and designing simulator environments?

R.Q.3. How does distributed cognition further our understanding of human-centered simulation?

R.Q.4. How can central theoretical simulation constructs be interpreted and explained through the distributed cognition perspective?

R.Q.5. What simulation components contribute to effective emergency medical management training according to the distributed cognition perspective?

\subsection{List of Publications}

To explore the aims and answer the research question, the following peer-reviewed publications are included in the thesis.

\section{Paper I: Real World Management Study}

Prytz, E., Rybing, J., Carlström, E., Khorram-Manesh, A., \& Jonson, C.-O. (2015). Exploring prehospital C2-work during a mass gathering event. International Journal of Emergency Services, 4(2), 227-241.

This paper presents an explorative study of inter-organizational management work, focused on medical management during a planned real-life major incident event. Ethnographical methods and questionnaires were used to explore cognitive processes and workload demands during emergency medical management activities, providing essential background knowledge for studies of cognitive processes in simulation.

My contribution: study design, fieldwork, data analysis, and writing.

Paper II: Applying Distributed Cognition to Study Simulation

Rybing, J., Nilsson, H., Jonson, C.-O., \& Bang, M. (2016). Studying distributed cognition of simulation-based team training with DiCoT. Ergonomics, 59(3), 423-434.

This paper presents a DiCoT analysis of the ETS training and assessment simulator for emergency medical management, demonstrating the use of distributed cognition for analyzing simulation environments. The analysis focuses on ETS from a distributed cognition perspective and how it creates a simulation environment in which cognitive aspects of emergency medicine management can be taught and assessed. In doing so, the paper makes connections between distributed cognition concepts and simulation validity. The paper also suggests benefits and drawbacks, using claims analysis, of a digital version of the ETS simulator based on the analysis.

My contribution: study design, fieldwork, data analysis, and writing.

Paper III: The Design of the DigEmergo Simulator 
Rybing, J., Prytz, E., Hornwall, J., Nilsson, H., Jonson, C.-O., \& Bang, M. (2017). Designing a Digital Medical Management Training Simulator Using Distributed Cognition Theory. Simulation \& Gaming, 48(1), 131-152.

This paper further connects distributed cognition theory to concepts of simulation validity by identifying core features and validity issues of ETS based on the DiCoT-analysis from Paper II. Describing validity using the language of distributed cognition demonstrates a "new discourse" for evaluating simulators. The paper further presents the digital simulator prototype (DIGEMERGO) based on the DiCoT analysis and an initial usefulness and usability study with subject-matter-expert users.

My contribution: prototype design, study design, system evaluation, data analysis, and writing.

\section{Paper IV: Preliminary Validation Results of the DigEmergo Simulator}

Rybing, J., Larsson, J., Jonson, C.-O., \& Prytz, E. (2016). Preliminary Validation Results of DigEmergo for Surge Capacity Management. In A. Tapia, P. Antunes, V. A. Bañuls, K. Moore, \& J. Porto (Eds.), Proceedings of the International ISCR AM Conference 2016. Rio de Janeiro, Brazil.

This paper presents initial results from a quantitative validation study of the DigEMERGo prototype based on user performance measures during a simulated emergency department surge capacity event. This study provides a direct test of simulation validity for the DigEMERGo prototype, and an indirect test of using DiCoT and distributed cognition for designing simulators.

My contribution: study design, data analysis, and writing.

\section{Paper V: Training with the DigEmERgo Simulator}

Jonson, C.-O., Pettersson, J., Rybing, J., Nilsson, H., \& Prytz, E. (2017). Short simulation exercises to improve emergency department nurses' self-efficacy for initial disaster management: Controlled before and after study. Nurse Education Today, 55(August), $20-25$.

In this paper, the DigEMERGo system is applied to train nurses' self-efficacy and management skills in managing a surge capacity event at a simulated emergency department. As in the previous paper, this paper is a test of validity, but rather than targeting internal validity the study targets educational validity in terms of training outcome and transfer of training.

My contribution: study design, prototype and scenario design, data analysis, and writing.

\section{Paper VI: Additional Validation of DigEmergo}

Rybing, J., Larsson, J., Jonson, C.-O., \& Prytz, E. (2018). Validation of a simulator for emergency medicine management skills. Journal paper manuscript.

The final paper presents addition results from the validation study of DigEMERGo presented in Paper IV. This paper expands the analysis to include workload measurements, additional performance measures, and discusses the appropriateness of the validation approach and the selected measures. In addition, the paper discuss how the validation approach relates to Feinstein and Cannon's (2002) dimensions of validity and introduces the cognitive fidelity concept.

My contribution: study design, data analysis, and writing.

\section{Supporting Publications}


Prytz, E, Rybing, J, Jonson, C-O, Petterson, A, Berggren, P, \& Johansson, B (2015) An exploratory study of a low-level shared awareness measure using mission-critical locations during an emergency exercise Proceedings of the Human Factors and Ergonomics Society Annual Meeting, 59(1), 1152-1156

Prytz, E, \& Rybing, J (2015) Evaluation of a novel method to study inter-organizational coordination in medical command and control centers In S J Stratton (Ed), 19th World Congress on Disaster and Emergency Medicine (WCDEM 2015). Capetown, South Africa, 21-24 April

Rybing, J, Prytz, E, Hornwall, J, Jonson, C-O, Nilsson, H, \& Bang, M (2015) Preliminary evaluation results of DigEmergo - a digital simulator prototype for disaster and emergency management training In S J Stratton (Ed), 19th World Congress on Disaster and Emergency Medicine (WCDEM 2015) Capetown, South Africa, 21-24 April

Prytz, PhD, E G , Rybing, MSc, J , \& Jonson, PhD, C-O (2016) Workload differences across command levels and emergency response organizations during a major joint training exercise Journal of Emergency Management, 14(4), 289-297

\subsection{Research Contributions}

This thesis includes both theoretical and technological contributions that pertain to both the simulation field and cognitive science - distributed cognition in particular. The thesis contributes new knowledge and adapts a new analytical framework for the simulation field. In doing so, the thesis extends distributed cognition to a new domain and a different purpose, thereby contributing to distributed cognition's usefulness and coverage. Below the contributions are divided into four sub-contributions.

C.1. An overview of terms used in simulation theory, especially those that relate to cognitive dimensions of simulators, is provided.

C.2. Exploration of the distributed cognition perspective for studying, evaluating, and designing human-centered simulators.

2.1. Application and demonstration of $\mathrm{DiCoT}$ as a methodological approach for evaluating and designing simulators.

2.2. A case study analysis of the Emergo Train System ${ }^{\circledR}$ simulator demonstrating that distributed cognition using DiCoT can be useful for evaluating simulators.

2.3. Design and implementation case study of DigEmergo simulator demonstrating that distributed cognition using DiCoT can be useful for designing simulators.

C.3. Interpretation of human-centered simulations as distributed cognitive systems.

3.1. Demonstration of the use of distributed cognition principles as discourse for interpreting and analyzing how cognitive processes relate to core simulation constructs, such as validity and fidelity.

3.2. Cognitive fidelity is suggested as a term that refers to the similarity between the cognitive system that is being simulated and the corresponding cognitive system in reality.

C.4. New knowledge and tools to enhance the domain of emergency medical management, especially for training and assessment.

4.1. Study and evaluation of the established ETS simulator. 
Chapter 1

4.2. Design, implementation, and initial usability, usefulness, and validation studies of a new digital simulator prototype, which can be used for training and assessment of emergency medicine management as well as a research tool for studies in emergency medicine.

\subsection{Assumptions}

This thesis makes a practical assumption: that the training simulator system that is being studied (i.e., ETS) is valid, functional, and useful. This assumption is based on the widespread international application and use of ETS. This is an important assumption from a technological research point of view. Developing and using simulation techniques to solve real world problems are forms of technological research, where the goal is for the technique in question (simulations in this case) to be functional and useful. Knowing whether the studied simulator actually is functional and useful is therefore critical. On the other hand, from a scientific point of view, this assumption is arbitrary, as it is the application of distributed cognition theory to describe and evaluate central properties of simulations that is of interest - not the studied simulators' inherent usefulness or functionality in and of itself.

\subsection{Reader's Guide}

Following this introduction, Chapter 2 provides the theoretical background upon which the results and conclusions of the thesis are built. The case domain background, emergency medical management and ETS, is presented in Chapter 3. Chapter 4 presents the research approach used in the thesis. In this chapter, the DiCoT methodology is described in detail. In the following two chapters, the collective results of the papers are presented and further built upon. Chapter 5 describes, in short, how distributed cognition was applied to study and evaluate ETS, and how this analysis led to the design of the DigEmergo simulator. Chapter 6 analyzes and re-interprets central constructs of human-centered simulation from a distributed cognition point of view. The final part of the thesis, Chapter 7, is a discussion of the findings and contributions of the thesis. Here, the research approach and results are discussed and future work is suggested. Finally, the chapter concludes with the contributions of the thesis. After the final chapter follows the bibliography of references used in the thesis and the constituent papers are appended in Appendix A. 


\section{CHAPTER 2 THEORETICAL BACKGROUND}

This chapter will provide a theoretical backdrop for the contributions and the research presented in the thesis. On this canvas, two disciplines of science are portrayed: the science of simulation and cognitive science ${ }^{1}$. Both fields are interdisciplinary in nature with overlapping related disciplines, among which we find computer science, human factors, artificial intelligence, philosophy, and psychology. Interestingly, the cognitive science and simulation disciplines have a shared history in that they both grew out of, or were radically transformed by, the explosive rise of computers and computer science in the second half of the 20th century $^{2}$ (C. M. Banks, 2009; Hutchins, 1995a; Klein, 2005; Sokolowski \& Banks, 2010; Wolfe \& Crookall, 1998). In the next sections, I will give a short overview of the two fields, starting with a description of simulation theory, with emphasis on human-centered simulations, and then moving on to cognitive science, especially the distributed cognition perspective.

\subsection{Simulations}

The word simulation comes from the Latin verb "simulare", meaning "to imitate" or "to behave as if". In WordNet, among the synonyms of the verb "simulate" we find words such as mimic, reproduce, and replicate (2017a). These words all point to the core idea of simulations: to copy something using something else. We also find words with negative connotations, such as to assume, fake, deceive, and misrepresent. Ironically, these words illuminate another important idea of simulations: that they are abstracted concepts that we assume resemble some real phenomenon, but the simulations themselves are in a sense fake or not real. It is important to recognize the twin nature of simulations, the simulation technology and the simulation science ${ }^{3}$. The first refers to using simulations as a tool to solve real-world problems and the latter to the study of the simulations themselves. Both endeavors are important for the purpose of the thesis, which is partly about contributing to the science of simulations using distributed cognition theory and partly about applying simulation techniques to emergency medical management training.

\subsubsection{A Brief History of Simulation}

The military were, and still are, probably the earliest and most influential users of simulation techniques. One of the earliest examples of (deliberate) simulation activities is wargames, a form of live battle roleplay in which the troops are divided into different teams that are to challenge and outwit each other on the battlefield (C. M. Banks, 2009). The Roman Empire (circa 500 B.C.E. - 1500 C.E.) is known to

\footnotetext{
${ }^{1}$ To avoid possible confusion, it should be noted that this thesis has nothing to do with the so called "simulation theory" that exists within cognitive science, which is a theory about understanding mental states of others 2 The official birthday of cognitive science has even been set in 1956, however both cognitive science and simulations can be traced further back in history than this

${ }^{3}$ To make things even more confusing, computer-simulations have also been called "the third branch of science", see (Pool, 1992)
} 
have conducted wargame training exercises that reflected the anticipated environment their troops would encounter when later facing the real enemy. Preparing the troops via simulation is believed to be one of the keys to the success of the Roman military (ibid). Other examples of early military simulations are abstract games. The chess family of games is believed to stem from the game Chaturanga, developed in the Gupta Empire (India) around the $6^{\text {th }}$ or $7^{\text {th }}$ century CE (C. M. Banks, 2009; Wolfe \& Crookall, 1998). The goal of the game that today is called chess is to checkmate the opponent's King by placing troops in such a way that the opponent cannot move their King. The chessboard is simulating a battlefield where two armies of equal strength meet with no environmental advantage on either side. The players are the military commanders and it is their tactical skill and cunning that will determine the outcome of the conflict.

Moving into the $20^{\text {th }}$ century, we come to one of the most iconic uses of simulation: aviation. Following the birth of the motor-powered airplane (the Wright Flyer I, 1903), the first recorded flight simulation appeared in 1921 (C. M. Banks, 2009). The Link flight simulator (or Link Pilot Maker) was a pilot trainer made to resemble an airplane, with wings and a fuselage mounted on a universal joint, which could make the simulated plane pitch and roll in response to the pilot using the controls (L3-Technologies, 2017). The US military used the simulator as a cost-effective way to train navy and army pilots. Amusement parks also bought the simulator, but for entertainment rather than training purposes. In the field of medicine, the first simulations were physical models of anatomy and verbal role-playing of patients. With the 1950s came the doll Resusci Annie, which marked a significant event in medical simulation history as the first training mannequin. Annie was the product of a collaboration between toy manufacturer Asmund Laerdal, Dr. Peter Safar, and Dr. Bjorn Lind. They originally developed the Resusci Annie for practicing mouth-to-mouth breathing, and following its successful application, Annie was later also used for cardiopulmonary resuscitation training (CPR; Cooper \& Taqueti, 2008; Drews \& Bakdash, 2013; K. R. Rosen, 2008). Interestingly, Annie's face came from the smiling death mask of L'Inconnue: The Unknown Woman of the Siene, who was an unidentified young woman found drowned in the River Seine (Leardal Medical, n.d.).

The introduction of the digital computer in the mid-20th century led to what Wolfe and Crookall (1998) have called the modern era of simulation. Computer-based simulation spread rapidly within scientific and engineering applications as many new types of simulations became possible (Klein, 2005). The simulation technique's growing importance was noted by Conway in the 1960s:

\section{"The use of a digital computer to perform simulated experimentation on a numerical model of a complex system is an increasingly important technique in many disciplines

$$
\text { today" (1963, p. 47) }
$$

For instance, in meteorology and nuclear physics, computer-based simulations were used as scientific tools to calculate and predict outcomes (Winsberg, 2015). The military used digital computers for simulated combat assessments (C. M. Banks, 2010). With the advancement of artificial intelligence (AI) research, more complex phenomena, such as human personality and behavior, could be simulated and the subfield of behavioral modeling emerged (C. M. Banks, 2009). The explosive growth of computer games for entertainment also had a significant impact on the use and development of simulations, as many computer games profited from certain elements of simulation. In healthcare, computercontrolled simulators began to appear in the late 1960s (Bewley \& O’Neil, 2013; Drews \& Bakdash, 
2013; K. R. Rosen, 2008). Most notable is the computerized patient mannequin Sim One that could breathe, had a heartbeat and blood pressure, was able to blink, could open and close its mouth, and could respond to administered drugs. Further advancement of computers has led to more complex and detailed simulators in medicine, and today highly advanced patient mannequins (see e.g., CEA HPS ${ }^{4}$ ) and full virtual environments (see e.g. Cohen et al., 2013) are used in medical training (Decker, Sportsman, Puetz, \& Billings, 2008; Grenvik \& Schaefer, 2004; K. R. Rosen, 2008; Seropian, 2003).

\subsubsection{The Simulation Discipline}

Simulations are used extensively to study the world. Since the late $20^{\text {th }}$ century, simulations have evolved beyond their role as a technique employed in other fields, and have become a full scientific discipline (Sokolowski \& Banks, 2010). Today, simulations are critical instruments of modern science used to generate knowledge by exploring models of the world (Klein, 2005; Padilla, Diallo, \& Tolk, 2011). Beyond scientific applications, simulations are found in many fields, such as engineering, military, management, medicine, education, gaming, and even in judicial court (Epstein, 2004; Ören, 2005). Simulation techniques are useful, as they enable research where real-world investigations would be difficult (or impossible) to conduct (Hollnagel, 2011; Sokolowski \& Banks, 2010). For example, the phenomena of interest could be inaccessible (black holes), too dangerous (nuclear meltdowns), too expensive (flight tests), or morally unacceptable (experimental treatment) to study empirically (C. M. Banks, 2009, 2010; Humphreys, 2004). Even though study of the real phenomena is often desirable, simulations provide a set of advantages in comparison to studying the real world, as they, for instance, allow for repeated observations, strict control of conditional parameters, and scalability.

The modern simulation discipline is also known as modelling \& simulation (M\&S). In an article analyzing M\&S, Padilla and colleagues (2011) categorize the discipline into three sub-disciplines: M\&S science, $M \& S$ engineering, and $M \& S$ applications. $M \& S$ science contributes to the theory of simulations, which defines the academic foundation of the discipline. $M \& S$ engineering applies theories of $M \& S$ science to find solution patterns for domain problems that can lead to generalized methods and approaches. M\&S applications develop specific simulation solutions to domain-specific problems, and these applications are often derived from problem domain expertise - not from $M \& S$ science or engineering. In categorizing this way, Padilla and colleagues (2011) indirectly make a distinction between technological research and basic science within the simulation discipline. In the next sections, we will look more closely at these two branches of simulation to better understand simulation research, frame theoretical concepts, and help evaluate the contributions of the thesis.

\subsubsection{Simulation as Technology}

Science and technology are highly interdependent activities, but distinct from each other (Brooks, 1994). A key difference between the technological and scientific sides of the simulation field lies in the resulting products of these activities. Science is about acquiring knowledge and truths about the world, whereas the goal of technology (technological research) is to build techniques that are functional and useful for real-world application (Nordin, 2017; Shapere, 1998). A technique is a broader concept than a physical object, it could also be a method or maneuver intended to help in solving practical problems. Also, science is (or should be) autonomous and independent in its pursuit of knowledge, whereas

\footnotetext{
${ }^{4}$ Commercially available Human Patient Simulator: https://caehealthcare com/patient-simulation/hps
} 
technological research is dependent on the needs and wants of external parties - the users who ultimately judge whether the techniques are successful or not. The application and development of simulations to generate domain knowledge and solve real-world problems is, therefore, a form of technological research. To understand what functional and useful techniques mean, let us examine the definitions of these concepts as suggested by Nordin (ibid).

\section{"A technique $T(E)$ functions (with respect to an intended result E) to a degree $r$ in situations of type $S$ if, and only if, (a) T(E) produces $E$ with at least the frequency (efficiency) $r$ in $S$, and (b) $r$ is greater than zero or what nature/ chance is doing by itself." (Nordin, 2017, p. 29)}

This definition claims that a technique is functional if it produces the intended results often enough in a given situation. Nordin argues that we can never prove or disprove that a technique will function regardless of the amount of testing. This is similar to the problem of induction: that no matter how many observations are made to support a theory or hypothesis, it cannot conclusively be proven true. No matter how many white swans are observed, it cannot be concluded and generalized that all swans are white; the next swan that swims by might well be black. What a successful test of a technique's functionality does prove, however, is that a technique can function; thus disproving the ultra-skeptical assumption that it never can function. Similarly to how the next swan might be black, the next test of a technique's functionality might prove successful despite any number of previously failed attempts.

For a technique to be useful, on the other hand, it must do more than simply function. For instance, a functioning technique that produces something irrelevant or unnecessary (without use) or that is impossible to operate (unusable) would not be useful. The context of use, the user group, and the technique's purpose are all essential for a technique's usefulness. Nordin (2017) provides a definition:

\section{"T(E) is useful (with respect to an intended result $\mathrm{E}$ ) if, and only if, there is at least one actual or potential user $x$ and (a) T(E) works sufficiently well and (b) $x$ views \\ T(E) as useful to him or her with respect to E." (Nordin, 2017, p. 62)}

Hence, according to Nordin, a technique can be considered useful if it can function and if there is an individual that can and wants to use it (perceives it as useful). Usefulness clearly relates to the concept of usability (Sharp, Rogers, \& Preece, 2007), as a technique that has such poor usability (or userfriendliness) that no user can or wants to use it cannot be considered useful. Whether a functioning technique is to be considered usable and useful is ultimately up to the users. To develop useful simulation techniques, it is therefore essential to understand the user and the context of use.

\subsubsection{Simulation as Science}

Science seeks truths about the world, which take the form of theories, hypotheses, and explanations outcomes that do not need usable applications (Okasha, 2002). Philosophically, theories, unlike techniques, are either true or false and only one true theory can exist that covers a certain phenomenon ${ }^{5}$. The science of simulation is about finding truths about simulations, which is not to be confused with using simulations as a technique to discover truths within other fields of science. According to Padilla and

\footnotetext{
${ }^{5}$ However, there might be multiple theories that are useful without being strictly true, for instance Newtonian physics
} 
colleagues (2011), the focus of study for M\&S science is "truth in models and simulations of a simuland" (phenomenon/system we want to study)"; and the main research question follows as "what is true within models and simulations?" According to those authors, $M \& S$ science is interested in theories that describe fundamental properties, mechanisms, and scope of simulations.

The scientific field of simulation is still young and not as stable and rigid as one would expect given the widespread use of simulations (Grune-Yanoff \& Weirich, 2010; Klein, 2005; Wolfe \& Crookall, 1998). The rapid growth of the field has resulted in incoherence with regard to what simulations are, how they should be used, studied, classified, evaluated, and designed, as well as which central concepts apply and how they should be defined (Cooper \& Taqueti, 2008; Feinstein \& Cannon, 2001; Feinstein, Mann, \& Corsun, 2002; Humphreys, 2004; Klein, 2005; Ören, 2011c; Ören \& Zeigler, 2012; Wolfe \& Crookall, 1998). The widespread use of simulations has focused on the problem domains of simulations, rather than the overarching theory of simulation (Padilla et al., 2011). This has resulted in many subfields of simulation, often with their own sets of terminology, methodologies, scientific perspectives, concepts, classifications, and validation paradigms, with little cross-referencing in-between (Cooper \& Taqueti, 2008). Klein phrases this problem as follows:

\section{"[...] there is a striking contrast between the technological sophistication that drives and is driven by computer simulation and the rudimentary, underdeveloped state of theory underpinning the simulation endeavor." (2005, p. 304)}

Several researchers have raised the question of what theoretical scientific framework - philosophy of science - (computer) simulation should adhere to (Feinstein \& Cannon, 2001; Klein, 2005; Kleindorfer, O’Neill, \& Ganeshan, 1998). For simulation researchers, showing (or proving) that a simulation actually replicates the real world is known as the problem of validation ${ }^{7}$ (Feinstein \& Cannon, 2003; Kleindorfer et al., 1998), which is similar to the problem of induction and the problem of functionality described previously. In response to this problem, Klein (2005) argues that simulation validation should adhere to the Popperian falsification framework of science. From this point of view, theories cannot be proven true (the next swan might be black), only corroborated. However, they can be falsified and rejected when contradictory evidence is found (i.e., observation of a black swan). This essentially means that simulations can never be proven valid (or true if we treat a simulation like a theory), successful validation can only corroborate the validity of the simulation. Validation can, on the other hand, definitively falsify a simulation.

Following Klein and a Popperian line of simulation validation leads to an interesting possible conflict between simulation as science and simulation as technological research. Testing the functionality of a technique can never conclusively disprove functionality according to Nordin (2017), but can demonstrate functionality. However, according to the Popperian framework, a simulation cannot be proven valid - but can be proven invalid. Does this mean that simulations are stuck in the middle, without the possibility of being either provable or disprovable? I think not. I believe this illustrates the twin nature the simulation discipline: as technological research and as science. To give an example. A simulation that is used to predict the outcome of a medical emergency response event can be falsified

\footnotetext{
${ }^{6}$ Padilla and colleagues use the term referent rather than simuland

${ }^{7}$ Methods for validation of simulations will be discussed in section 434
} 
if its predictions are found to be completely inaccurate - this would consequently be an invalid simulation. In other words, the theory of emergency management - which the simulation embodied proved faulty. However, if the predictions are accurate, it is still possible that the simulation (and its embodied theory) is invalid and simply happened to generate accurate results. This is the argument that a simulation cannot be concluded valid. However, the same simulation can still be considered functioning ${ }^{8}$ as a technique if it produces its intended outcomes (correct predictions). It can even be considered useful if its results can be used to solve the problem it was intended for. This also highlights that truth and functionality are different concepts, and simulations seem to position themselves somewhere in between the two.

\subsubsection{Central Concepts and Definitions}

Defining the concept simulation is difficult and there are numerous suggested definitions in the published literature. Before presenting the definition that will be used in this thesis, this section will first decompose the activity of simulation into four central theoretical concepts: simulands, referents, models, and simulators. These will be introduced and defined one by one, after which a definition of simulation will be given. Figure 1 illustrates how these concepts relate to each other. The relationships between the concepts will be further explained as the concepts are presented.

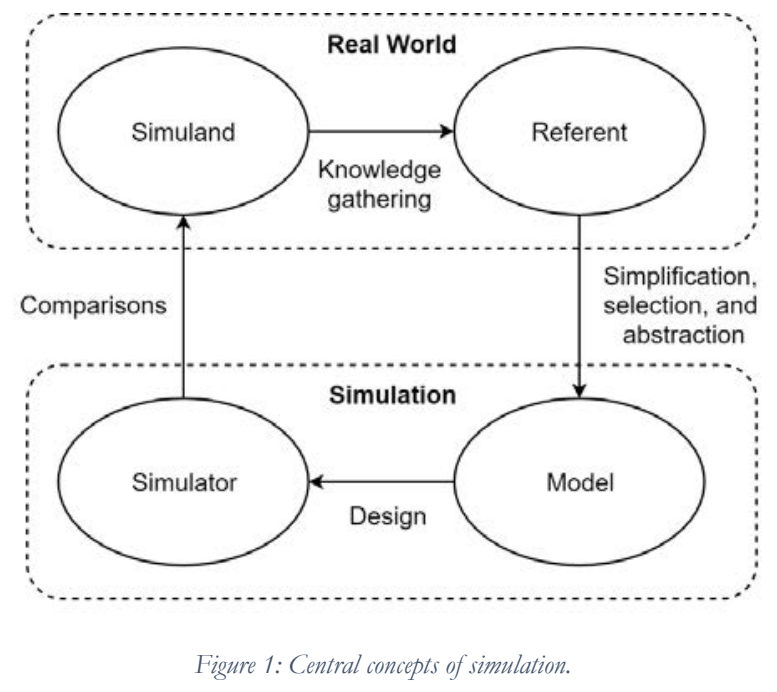

\section{Simulands and Referents}

The first two concepts represent what is to be simulated and our understanding of such phenomena. The simuland refers to the real-world items that we wish to simulate (Sokolowski \& Banks, 2010). These can be physical objects, such as airplanes or medical patients; processes, such as patient flow through a hospital; or a phenomenon, such as the weather or the movement of planetary objects in space. The simuland usually encompasses additional factors that significantly affect the primary items of interest; for instance, the simuland of a flight simulation would probably include weather phenomena and not just the airplane. The referent is the knowledge about the simuland that is available to the simulation

\footnotetext{
${ }^{8}$ The simulation would still be considered functional even though is underlying theory of management is faulty, as long as the simulation - for some reason - predicts accurate outcomes
} 
researcher or modeler - the person(s) who is to model the simuland and design and construct the simulation. The referent can include both quantitative formal knowledge, such as physics equations, statistics, or empirical data; and qualitative knowledge, such as scientific theories, empirical observations, or personal experience of the simuland.

\section{Models}

Central to simulation theory is the notion of a model of reality that underlies the simulation. In simulation theory, a model is a simplified, selective, and abstracted physical, mathematical, or logical (conceptual) representation of the simuland9 (Crookall, Oxford, \& Saunders, 1987; Sokolowski \& Banks, 2010). Every scientific field uses models more or less formally and knowingly. For instance, in computer science, structured models (e.g., using the modeling language $\mathrm{UML}^{10}$ or mathematical equations) are frequently used to communicate and analyze software. In the field of anthropology, the textual and descriptive interpretations that are used to characterize the unit of analysis (cultural phenomenon) are also types of models. For computer-based simulation, a broad distinction is often made between conceptual models and executable models (Sargent, 2012; Sokolowski \& Banks, 2010). Conceptual models can be seen as all forms of descriptive documentation of the simuland (e.g., UML diagrams, interface designs, requirements lists, flow charts, or mathematical formulas), whereas an executable model is the implemented software code that can be executed (run) by a computer.

Models are separate from scientific theories (Humphreys, 2004). A fundamental distinction between the two is that theories aim to be true and models do not. This is captured by George E.P. Box's aphorism: "all models are wrong, but some are useful" (first used in Box, 1976). For instance, the atom model proposed by Bohr (1913) is a simplification and abstraction of a real atom, and thus not true - but it's still a concept that has proven to be useful. In contrast, the theory of heliocentrism, as suggested by Copernicus, states that the Earth revolves around the sun. This is not a simplification nor an abstraction, but rather a truth about the world as we understand it. All models embody some theory about the system they describe (Crookall et al., 1987). Often, models are said to be located somewhere between reality and theory as a mediator (Grune-Yanoff $\&$ Weirich, 2010). In a sense, a model is itself a type of technique we can employ to simplify the world so that we can understand it. From this line of thought, it follows that simulations are not true either, but rather more or less useful techniques for making sense of the world around us. Thus, simulations should not to be treated as scientific theories in any strict sense.

\section{Simulators}

A simulator is the device that somehow executes the model to produce the simulation process. Here we can use the definition from Hancock and colleagues (2010), where they characterize a simulator as "a setting, device, computer program or system that performs simulation". In most cases, the distinction between simulation and simulator is clear. For instance, in a computer-based simulation with no human interaction as part of the simulation, the simulator consists of the hardware and the software (the executable model). The simulation takes place when the simulator executes its model - in other words, when someone pushes the start button. In this example, the simulator was easy to identify. However,

\footnotetext{
${ }^{9}$ To be precise, a model is really a representation of a referent, rather than the real word, as we model based on our understanding of the world

${ }^{10}$ Unified Modeling Language, see http://www uml org/
} 
occasionally the simulator can be difficult to distinguish from the simulation activity. For instance, what do we make of role-play style simulations where groups of people mimic the behavior of some target system or situation (e.g., war-games or simulated patient interaction)? Either the group of people is the simulator or there is no formal simulator, only simulation. In this thesis the first alternative is preferred, as the group of people can be seen as a system that realizes (or executes) a shared model of reality, which fits Hancock et al's definition of a simulator.

\section{Simulations}

With all the bits and pieces in place, defining the actual simulation is still problematic (Ören, 2011b, 2011c; Sokolowski \& Banks, 2010; Winsberg, 2015). One reason for this is that the multitude of applications has led to diverse conceptualizations of simulation. To illustrate this, Ören (2011b, 2011c) collected over 100 definitions of simulation. Some definitions consider simulations as a method for executing a model, some emphasize the intended purpose, while others compare simulations to traditional experiments (Grune-Yanoff \& Weirich, 2010). Additionally, many of the definitions of simulation only consider computer-based simulation. Table 1 shows a few suggested definitions from the literature.

Table 1: Examples of suggested definitions

\section{Definition}

"The process of designing a model of a real system and conducting experiments with this model for the purpose either of understanding the behavior of the system or of evaluating various strategies (within the limits imposed by a criterion or a set of criteria) for the operation of the system "

"The representation of the dynamic behavior of the system by moving it from state to state in accordance with well-defined operating rules "

"Simulation is just one of the techniques in the field of operations research and management science (OR/MS)"

"A simulation imitates one process by another"

"Simulation is the imitation of the operation of a real-world process or system over time"

"A method for implementing a model over time "

"The act of imitating the behavior of some situation or some process by means of something suitably analogous (especially for the purpose of study or personnel training)"

"Act of pretending; imitation, counterfeit; imitation of the operation or features of one system using another system (i e computer simulation)"

\section{Reference}

Shannon 1975 in Ören, 2011c

Pritsker and Pegden 1979 in Ören, 2011c

Screiber 1991 in Ören, 2011c

Hartmann, 1996, p 77

J Banks, Nelson, Carson, \& Nicol, 2014

DoD M\&S Glossary, 2010

Princeton University, 2017b

Princeton University, 2017b

Winsberg (2015) attempts to summarize the definitions from a philosophical viewpoint. He claims that computer simulations are frequently defined in a narrow or a broad sense, where the former emphasizes the algorithmic properties of simulation, and the latter incorporates the entire process of simulation: modeling, implementation, and assessing output. Winsberg widens the concept by providing an alternative definition, where he claims that a simulation is: 


\section{"[...] any system that is believed, or hoped, to have dynamical behavior that is similar enough to some other system such that the former can be studied to learn about the \\ latter." (Winsberg, 2015, p. 3 emphasis added)}

This is the definition of a simulation that will be used throughout this thesis ${ }^{11}$. Figure 2 provides an illustration of this definition combined with the core simulation concepts. According to the definition, a simulation consists of the following components: the any system (the simulator), the other system (the simuland), and the dynamic behavior produced by the any system (the simulation processes produced by the simulator). Furthermore, the generated behavior must fulfill the condition of being similar enough to that of the simuland for a simulation to take place. This similar enough condition is problematic as it is relative. The behavior of a vacuum cleaner robot is probably not similar enough to that of a wild tiger for it to be considered a simulation of one; however, what if we program the robot to stalk and pounce on unsuspecting house pets? Does this change in behavior make it similar enough? A solution to this problem is provided by the definition, as it states that the behavior is similar enough if we can learn about the targeted other system through studying the any system. This is a question of validating the simulation and its output (see section 4.3.4.1). Thus, if we can learn about tigers by studying the vacuum cleaner it can indeed be considered a simulation of tigers. A consequence of this definition is that a system is really a simulation of another system once it has been demonstrated that we can learn from it, in other words after it has been validated.

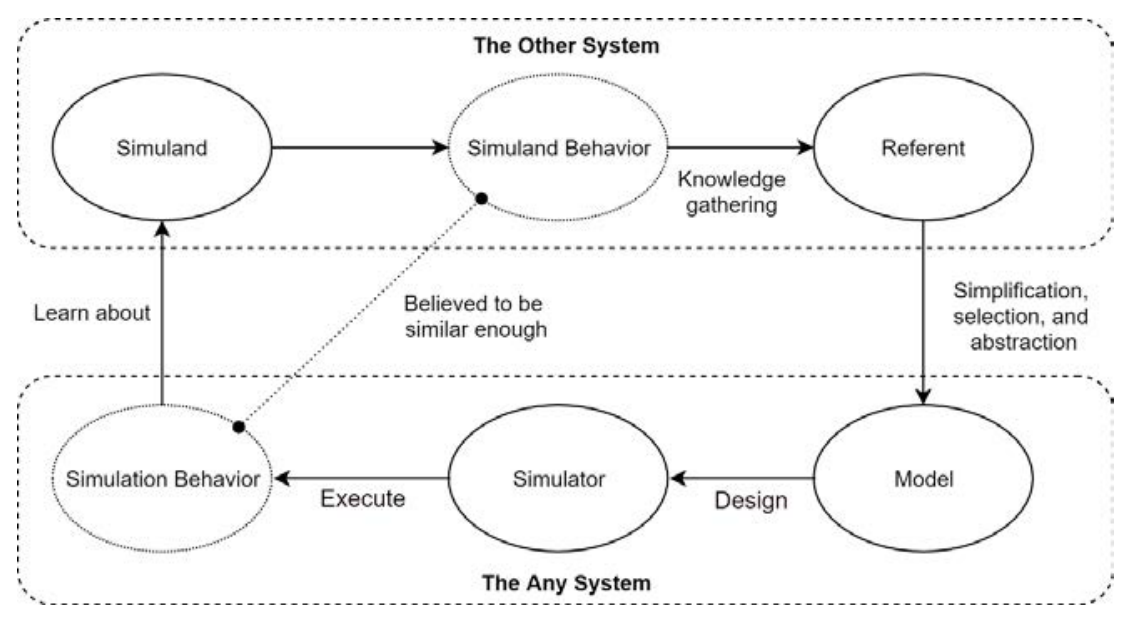

Figure 2: Simulation concepts enriched with Winsberg's (2015) definition of a simulation.

There is something further to note in the definition: that the any system is believed, or hoped, to behave similarly enough to the other system. This is an interesting choice of words, as many definitions would rather claim that the simulator is modeled or intended to mimic the simuland. Winsberg's definition leaves intent out of the picture. Returning to the example with the tiger, this phrasing allows an ordinary house cat to be considered as a simulation of a tiger, even though no one has designed the cat with the intent

11 This definition is not without its problems For one, it is argued, for instance by Grune-Yanoff and Weirich (2010), that simulations do not necessarily have to be dynamic Also, Monte Carlo simulations are an example of simulations that do not have a mimetic purpose or approach, but they are still considered simulations However, these issues are of no concern for the simulations that are addressed in this thesis 
of simulating a tiger; and surely, a cat would be a more fitting simulation of a tiger than the modified vacuum cleaner robot. Crookall shares this notion:

\section{"[...] anything can simulate anything else; simulation is all in the mind, not in some intrinsic quality of the objects that we employ in the simulator."}

$$
\text { (Crookall, 2010, p. 904) }
$$

In summary, the simuland refers to what things in real-life is to be simulated and the referent is the knowledge about those things. Based on the referent, abstractions, simplifications, and selections of the simuland are made to form a model. This model is executed by some unit- the simulator (e.g., a computer) - and this process of execution generates the simulation behavior, which can be studied to learn about the simuland.

\subsubsection{Simulation Classification}

There are multiple simulation classifications in the published literature. Often classifications are bound to specific application domains, see for instance Gaba (2004). There are also classifications bound to application purposes, such as classifications of simulations specifically used for training. A combination of the two is found in Beaubien and Baker (2004), who classify training simulators in healthcare into three categories: role-play and case studies, part-task trainers, and full mission simulations. In this thesis, a coarse classification of simulations in general will be used, which categorizes simulations as live, virtual, or constructive (Andrews, Brown, Byrnes, Chang, \& Hartman, 1998; Sokolowski \& Banks, 2010). Live simulations involve real people that operate real equipment to replicate some real situation. Military war-games and full-scale emergency medicine simulations using actors are examples of live simulation. In virtual simulations, real people interact with simulated tools and environments - this is perhaps the most common type of simulation. Virtual simulations include a variety of simulators, such as vehicle cockpit simulators (e.g., the Link simulator), virtual and augmented reality (VR and AR), microworlds (e.g. C3-Fire; Granlund, 2017), and various types of role-playing simulations (see e.g. Chang et al., 2015). They also include part-task simulators, which are simulators that simulate parts of the targeted system for training purposes, such as medical mannequins. Part-task simulators are numerous but all follow the same basic principle: that they are designed to segment a complex task into its main components (Beaubien \& Baker, 2004).

Constructive simulations are autonomous, and they simulate all involved systems, equipment, and interacting people. Contrary to the other two categories, real people do not affect the outcome of the simulation, except for providing input by setting up initial parameters and configurations. Constructive simulations come in many forms; two of the most common are discrete event simulations and Monte Carlo simulations (C. M. Banks, 2010; Sokolowski, 2010). Constructive simulations can be used, for instance, to simulate planetary movements of the galaxy or patient flows at hospital wards to predict medical strain (see e.g. Hirshberg, Frykberg, Mattox, \& Stein, 2010). Moreover, Andrews et. al. (1998) argue for a fourth class, bybrid simulation, in which two or more of the above three types of simulations are combined.

Human-centered simulations are either live or virtual simulations in which humans play a central role, either by being part of the simulator (e.g., role-playing) or through interaction with the simulator (e.g., using a medical mannequin). Traditionally in simulation research, human interaction has been viewed 
as a mere source of system input (Rothrock \& Narayanan, 2011), which is different from humancentered simulations where humans are central parts of the simulation. Human-in-the-loop simulations and interactive simulations (Narayanan \& Kidambi, 2011) are two related concepts found in the simulation literature. These refer to simulations where one or more human operators are in direct control of simulation functions. A difference between the human-centered simulation and the human-in-the-loop concept is that the latter emphasize computer-based-simulation, whereas the former does not.

\subsubsection{Simulation Constructs}

With an understanding of simulation components and a broad classification in place, it is time to dissect the core constructs of simulations used in the simulation discourse.

\subsubsection{Descriptive Attributes of a Simulation}

According to Banks (2010), simulations in general can be described primarily by three attributes: fidelity, resolution, and scale. In this section, we will focus on fidelity, as it is the one most relevant for the thesis. However, I will start by briefly describing the other two. Resolution describes the degree of detail included in the simulation. A greater amount of detail, in terms of the amount of referent information included in the conceptual model, means higher resolution (C. M. Banks, 2010). The scale property describes the size of the simulation. Scale has to do with where the boundaries of a simulation should be drawn, in terms of which objects or phenomena are to be conceptually modeled, to reach its aims (C. M. Banks, 2010). For example, a large-scale medical simulation could include the entire ward or the entire hospital, not just single patients. Large scale usually implies low resolution, and vice versa, due to the required computational power and effort it takes to model and execute large-scale high-resolution simulations.

Fidelity is, in essence, a description or measure of how closely the simulation matches reality. In other words, it is the perceived realism of the simulation (C. M. Banks, 2010). A simulation that is a complex and lifelike replica of the simuland would be considered a high-fidelity simulation - something that looks or feels like the real thing. A simulation with "perfect fidelity" would be identical to the simuland (Drews \& Bakdash, 2013), and a low-complexity version, such as a pilot cockpit made out of cardboard, would be considered low-fidelity. We can use Hays and Singer's definition of fidelity for training simulators as a general definition, which states that fidelity is:

\section{"[...] the degree of similarity between the [training] situation and the operational situation which is simulated". (Singer \& Hayes, 1989, p. 50)}

Fidelity is not to be confused with resolution. A simulation can have high resolution and low fidelity (C. M. Banks, 2010). For instance, consider a medical simulation of a patient that includes detailed referent information: all medical history, personal information, and all body and health information. This would be considered a high-resolution simulation. At the same time, the patient and all her details can be simulated using a low-fidelity approach, for instance through sketches on paper or using a simple physical doll supplemented with detailed patient charts. No one would confuse this patient simulation with a real human being even though it has a high resolution.

When the term fidelity is used alone in the literature (i.e., when referring to a simulation as having high or low fidelity) it refers to physical fidelity, which is the degree to which the simulation replicates sensory 
characteristics of the simuland (Drews \& Bakdash, 2013). Fidelity has also been used to describe many other resemblances to reality, such as behavioral resemblance, similarity in tasks, subjective experience, functional resemblance, etc. See Table 2 for a short summary of fidelity terms used in the literature.

Table 2: Examples of fidelity types from the literature.

\begin{tabular}{|c|c|c|}
\hline Fidelity & Description & Reference \\
\hline Action fidelity & $\begin{array}{l}\text { "[...] relations between performance in the } \\
\text { simulator and performance in the simulated } \\
\text { system" }\end{array}$ & $\begin{array}{l}\text { Stoffregen, Bardy, Smart, \& } \\
\text { Pagulayan, } 2003\end{array}$ \\
\hline Cognitive fidelity & $\begin{array}{l}\text { "[...] refers to the extent that the simulator engages } \\
\text { the trainee in the types of cognitive activities } \\
\text { involved in the real-world task" }\end{array}$ & Tsang \& Vidulich, 2002 \\
\hline $\begin{array}{l}\text { Environment } \\
\text { fidelity }\end{array}$ & $\begin{array}{l}\text { "The degree to which the simulator duplicates } \\
\text { sensory information from the task environment" }\end{array}$ & Beaubien \& Baker, 2004 \\
\hline Equipment fidelity & $\begin{array}{l}\text { "The degree to which the simulator duplicates the } \\
\text { appearance and feel of the real system" }\end{array}$ & Beaubien \& Baker, 2004 \\
\hline $\begin{array}{l}\text { Experiential } \\
\text { fidelity }\end{array}$ & $\begin{array}{l}\text { "[...] is the extent to which a simulation gives rise } \\
\text { to a subjective experience of "being there" } "\end{array}$ & Stoffregen et al , 2003 \\
\hline Functional fidelity & $\begin{array}{l}\text { "[...] describes the degree to which the simulation } \\
\text { reacts like "the real thing", } \mathrm{i} \text { e, that it provides } \\
\text { realistic responses on the tasks and actions executed } \\
\text { by the participant" }\end{array}$ & Dahl, Alsos, \& Svanæs, 2010 \\
\hline $\begin{array}{l}\text { Physical, } \\
\text { Engineering } \\
\text { fidelity }\end{array}$ & $\begin{array}{l}\text { "[...] the degree to which the physical } \\
\text { characteristics of the system are replicated within } \\
\text { the simulation" }\end{array}$ & $\begin{array}{l}\text { Drews \& Bakdash, 2013; R B Miller, } \\
1954\end{array}$ \\
\hline $\begin{array}{l}\text { Psychological } \\
\text { fidelity }\end{array}$ & $\begin{array}{l}\text { "[...] the degree to which the trainee perceives the } \\
\text { simulation to be a believable surrogate for the } \\
\text { trained task" }\end{array}$ & R B Miller, 1954 \\
\hline Task fidelity & $\begin{array}{l}\text { "[...] describes the degree to which tasks involved } \\
\text { in the actual environment for a given domain is } \\
\text { replicated in the simulation" }\end{array}$ & Rehmann, Mitman, \& Reynolds, 1995 \\
\hline
\end{tabular}

Miller was the first to make the distinction between physical fidelity and psychological fidelity (Miller 1953 cited in Maran \& Glavin, 2003). Psychological fidelity (also called cognitive fidelity; Liu, Macchiarella, \& Vincenzi, 2008), is the degree to which the simulation recreates the underlying psychological processes (e.g., communication, decision-making, planning, etc.) involved in the simuland phenomena or activity. Kozlowski and DeShon describe the difference between physical and psychological fidelity in their discussion of the way the two "approaches" try to capture central constructs and mechanisms that enable human performance:

\section{"Whereas the physical fidelity approach attempts to accomplish this implicitly by replicating the performance environment, the psychological fidelity approach represents an \\ effort to model this explicitly by using basic theory to guide research and training design." (2004, p. 4)}

Often it is physical fidelity that is emphasized and prioritized in simulation, and something that is inherently strived for (Hamstra, Brydges, Hatala, Zendejas, \& Cook, 2014). However, research has found this preference for high fidelity to be problematic, as (overly) high fidelity is both expensive and not always necessary - or beneficial - for a simulation to achieve its purpose, especially for training applications J. A. Adams, 1979; Alessi, 1988; Beaubien \& Baker, 2004; Dahlstrom, Dekker, Van 
Winsen, \& Nyce, 2009; Dieckmann, Gaba, \& Rall, 2007; Drews \& Bakdash, 2013; Salas, Bowers, \& Rhodenizer, 1998; Scerbo \& Dawson, 2007; Singer \& Hayes, 1989; Smallman \& John, 2005). Smallman and St. John (2005) referred to the preference for high physical fidelity as naïve realism, by which they mean that high fidelity (in their case realistic visual graphics) is preferred over low fidelity (less realistic graphics) even when the less realistic display leads to a better understanding of the information. The following quote describes the driving force behind the pursuit of high-fidelity simulations:

\section{"[...] by brute engineering force all physical and cognitive aspects of a task can be captured" (Caird, 1996, p. 127).}

That is, by making physical realism high enough, all aspects of reality will be captured and thus the "similar enough" criteria from the simulation definition would be fulfilled.

Several researchers emphasize the importance of psychological fidelity in training simulations (Drews \& Bakdash, 2013; Kozlowski \& DeShon, 2004), and it has been shown that high psychological fidelity does not necessarily require high physical fidelity (Drews \& Bakdash, 2013; Hamstra et al., 2014; Maran \& Glavin, 2003). Rather, recreating aspects like social and team work structures seem to contribute to psychological fidelity (Bratzke, Downs, \& Smith, 1998; Ker, Mole, \& Bradley, 2003; Maran \& Glavin, 2003). In contrast, Hontvedt (2015) demonstrated how expert simulation participants' perception of their physical work environments ${ }^{12}$ came into conflict with a (too) low-fidelity simulated environment. In this case, high physical fidelity was required to mediate the experts' psychological processes. This is in line with Alessi (1988), who recognized that expert users might require higher levels of fidelity to be challenged in simulation. Fidelity of training simulations will be discussed further in section 2.1.4.3.

\subsubsection{Validity Concepts}

According to Banks (2010), fidelity is occasionally used incorrectly in the literature to express the accuracy or correctness of the representations used in a simulation. This is instead an aspect of validity, which is another key concept in simulation theory (Bewley \& O'Neil, 2013; Feinstein \& Cannon, 2001, 2002; Pace, 2004; Peters, Vissers, \& Heijne, 1998; Sargent, 1996). In general, validity deals with how well the simulation fits the simuland in the context of the simulation purpose. What this means specifically is difficult to define, as validity is a multifaceted concept. According to Banks (2010), it refers to quality of modeling; and according to Padilla et al. (2011), it is established by comparing simulation results to data acquired in reality. It seems that validity can be viewed both as an aspect of a simulation's construction and of its output, allowing for a means of demonstrating that the simulation can be used as intended (Bewley \& O’Neil, 2013; Pace, 2004; Sargent, 2012). Validity is always related to purpose and intended use (Pace, 2004). To address the multitude of validity concepts, Feinstein and Cannon (2002) composed a list of 19 concepts that relate to simulation validity. This list has been adopted and further extended with additional validity characteristics in Table 3.

\footnotetext{
12 This is known as "professional vision" (Goodwin, 1994)
} 
Table 3: Concepts related to validity found in the literature. The table is adopted from Feinstein and Cannon (2002) and extended with further concepts.

\begin{tabular}{|c|c|c|}
\hline $\begin{array}{l}\text { Validity } \\
\text { Concept }\end{array}$ & Validity Question or Definition & Reference \\
\hline $\begin{array}{l}\text { Absolute } \\
\text { validity }\end{array}$ & $\begin{array}{l}\text { Is there a numerical match in measures of the dependent variable } \\
\text { between simulation and reality in response to the independent } \\
\text { variables? }\end{array}$ & $\begin{array}{l}\text { Blauw } 1982 \text { cited in Carsten } \\
\text { \& Jamson, } 2011\end{array}$ \\
\hline Accuracy & $\begin{array}{l}\text { Does a simulation game accurately mirror the reality it is supposed to } \\
\text { represent? }\end{array}$ & Dukes \& Waller, 1976 \\
\hline $\begin{array}{l}\text { Algorithmic } \\
\text { validity }\end{array}$ & Does the model return appropriate values? & Wolfe \& Jackson, 1989 \\
\hline $\begin{array}{l}\text { Behavioral } \\
\text { validity }\end{array}$ & $\begin{array}{l}\text { How closely does the experience and the behavior of the participant } \\
\text { approximate that in the real-life task? }\end{array}$ & Carsten \& Jamson, 2011 \\
\hline Believability & $\begin{array}{l}\text { Does the simulation model's ultimate user have confidence in the } \\
\text { model's results? }\end{array}$ & $\begin{array}{l}\text { Pegden, Sadowski, \& } \\
\text { Shannon, } 1995\end{array}$ \\
\hline $\begin{array}{l}\text { Conceptual } \\
\text { validity }\end{array}$ & Does the model adequately represent the real-world system? & $\begin{array}{l}\text { McDougall, 2007; Pegden et } \\
\text { al , } 1995\end{array}$ \\
\hline $\begin{array}{l}\text { Concurrent } \\
\text { validity }\end{array}$ & $\begin{array}{l}\text { To what extent does the simulator, as an assessment tool, correlate } \\
\text { with a "gold standard"? (e g, OSATS scores for surgery; part of } \\
\text { criterion validity) }\end{array}$ & McDougall, 2007 \\
\hline \multirow[t]{2}{*}{$\begin{array}{l}\text { Construct } \\
\text { validity }\end{array}$} & $\begin{array}{l}\text { How correctly are the variables in the model related to each other to } \\
\text { form strategic and environmental constructs? }\end{array}$ & Babbie, 1992 \\
\hline & $\begin{array}{l}\text { Is the simulator able to distinguish the experienced from the } \\
\text { inexperienced surgeon? }\end{array}$ & McDougall, 2007 \\
\hline $\begin{array}{l}\text { Content } \\
\text { validity }\end{array}$ & $\begin{array}{l}\text { How complete is the simulation model, relative to the demands } \\
\text { imposed by the purpose for which the model was developed? }\end{array}$ & Babbie, 1992 \\
\hline $\begin{array}{l}\text { Convergent } \\
\text { validity }\end{array}$ & $\begin{array}{l}\text { How well do simulation performance results compare with other } \\
\text { measures of comparable competencies? }\end{array}$ & Cannon \& Burns, 1999 \\
\hline $\begin{array}{l}\text { Criterion } \\
\text { validity }\end{array}$ & $\begin{array}{l}\text { How do evaluation results from the new simulator correlate to those of } \\
\text { the old technique or evaluation ( } \mathrm{ie} \text {, the simuland)? }\end{array}$ & McDougall, 2007 \\
\hline $\begin{array}{l}\text { Ecological } \\
\text { validity }\end{array}$ & $\begin{array}{l}\text { To what degree is the relation between proximal cues and the } \\
\text { dimensions of distal objects that they represent accurate? }{ }^{14}\end{array}$ & $\begin{array}{l}\text { Brunswik } 1955 \text { cited in } \\
\text { Hoffman \& Deffenbacher, } \\
1993\end{array}$ \\
\hline $\begin{array}{l}\text { Educational } \\
\text { validity }\end{array}$ & $\begin{array}{l}\text { Does the simulation provide a valid learning experience and/or } \\
\text { assessment of learning? }\end{array}$ & Feinstein \& Cannon, 2002 \\
\hline $\begin{array}{l}\text { Empirical } \\
\text { validity }\end{array}$ & $\begin{array}{l}\text { Does a simulation [game] exhibit a closeness of fit to other measures } \\
\text { of the phenomena it is designed to simulate? }\end{array}$ & Boocock, 1972 \\
\hline Event validity & $\begin{array}{l}\text { The degree to which a simulation's predicted responses correspond to } \\
\text { actual data from the [organization] being simulated }\end{array}$ & Mihram, 1972 \\
\hline
\end{tabular}

\footnotetext{
${ }^{13}$ Carsten and Jameson use the definition in a driving simulator context, and thus use the terms driving and vehicle rather than behavior and task

${ }_{14}$ This concerns the fact that the distance between objects should be represented in a way that reflects the corresponding distance in reality Ecological validity has more recently evolved to refer to the degree to which a given stimulus in experiments has features that correspond to naturally occurring stimuli, for instance in experimental materials, tasks, and conditions (Hoffman \& Deffenbacher, 1993)
} 


\begin{tabular}{|c|c|c|}
\hline $\begin{array}{l}\text { Extemal } \\
\text { validity }\end{array}$ & Does the simulation model represent actual external phenomena? & $\begin{array}{l}\text { Cook and Campbell } 1979 \\
\text { cited in Feinstein \& Cannon, } \\
2002\end{array}$ \\
\hline Face validity & $\begin{array}{l}\text { Relates to the realism of the simulator; does the simulator represent } \\
\text { what it is supposed to represent? }\end{array}$ & McDougall, 2007 \\
\hline $\begin{array}{l}\text { Internal } \\
\text { validity }\end{array}$ & Do a model's relationships represent true causality? & $\begin{array}{l}\text { Cook and Campbell } 1979 \\
\text { cited in Feinstein \& Cannon, } \\
2002\end{array}$ \\
\hline $\begin{array}{l}\text { Operational } \\
\text { validity }\end{array}$ & $\begin{array}{l}\text { Are the model-generated behavioral data characteristic of the real- } \\
\text { world system's behavioral data? }\end{array}$ & $\begin{array}{l}\text { Pegden et al , 1995; Sargent, } \\
1996\end{array}$ \\
\hline $\begin{array}{l}\text { Physical } \\
\text { validity }\end{array}$ & $\begin{array}{l}\text { How closely do physical components and subsystems of the simulator } \\
\text { approximate the real-life components? }\end{array}$ & Carsten \& Jamson, 2011 \\
\hline Plausibility & Does the simulation model appear to represent real-life phenomena? & Boocock, 1972 \\
\hline $\begin{array}{l}\text { Predictive } \\
\text { validity }\end{array}$ & $\begin{array}{l}\text { Does the model effectively predict real-world situations? (part of } \\
\text { criterion validity) }\end{array}$ & $\begin{array}{l}\text { Babbie, 1992; McDougall, } \\
2007\end{array}$ \\
\hline Realism & $\begin{array}{l}\text { Does the simulation represent the [business] environment it is designed } \\
\text { to simulate? }\end{array}$ & Norris, 1986 \\
\hline $\begin{array}{l}\text { Relative } \\
\text { validity }\end{array}$ & $\begin{array}{l}\text { Do the measures of dependent variables follow the same "pattern" } \\
\text { ( } \mathrm{g} \text {, direction and amplitude) in response to the independent variables } \\
\text { as it would in reality? }\end{array}$ & $\begin{array}{l}\text { Blauw } 1982 \text { cited in Carsten } \\
\text { \& Jamson, } 2011\end{array}$ \\
\hline $\begin{array}{l}\text { Representation } \\
\text { al validity }\end{array}$ & $\begin{array}{l}\text { Does the simulation provide a valid representation of a desired } \\
\text { phenomenon? }\end{array}$ & Feinstein \& Cannon, 2002 \\
\hline $\begin{array}{l}\text { Structural } \\
\text { validity }\end{array}$ & $\begin{array}{l}\text { Requires that the model both reproduces the observed system behavior } \\
\text { and also truly reflects the way in which the real system operates to } \\
\text { produce this behavior }\end{array}$ & $\begin{array}{l}\text { Grune-Yanoff \& Weirich, } \\
\text { 2010; Raser } 1969 \text { cited in } \\
\text { Peters et al , } 1998\end{array}$ \\
\hline $\begin{array}{l}\text { Verisimilitude } \\
\text { (face-validity) }\end{array}$ & $\begin{array}{l}\text { Does the simulation model appear to represent real-life phenomena? } \\
\text { Often referred to as face validity }\end{array}$ & Kibbee, 1961 \\
\hline
\end{tabular}

Feinstein and Cannon (2001, 2002) categorized and re-interpreted validity concepts of SBT simulations ${ }^{15}$ roughly onto four basic concepts of validity. representational validity, educational validity, internal validity, and external validity. The first two concepts address the development and application processes of a simulation, and the latter two relate to the structure and function of a simulation. Figure 3 below presents an overview of these four concepts as they are described by Feinstein and Cannon (2002). These validity concepts frequently overlap and can be hard to separate, but together they form a framework for interpreting the multifaceted topic of simulation validity.

${ }^{15}$ Feinstein and Cannon (2002) use the terms "game" and "simulation" somewhat interchangeably 


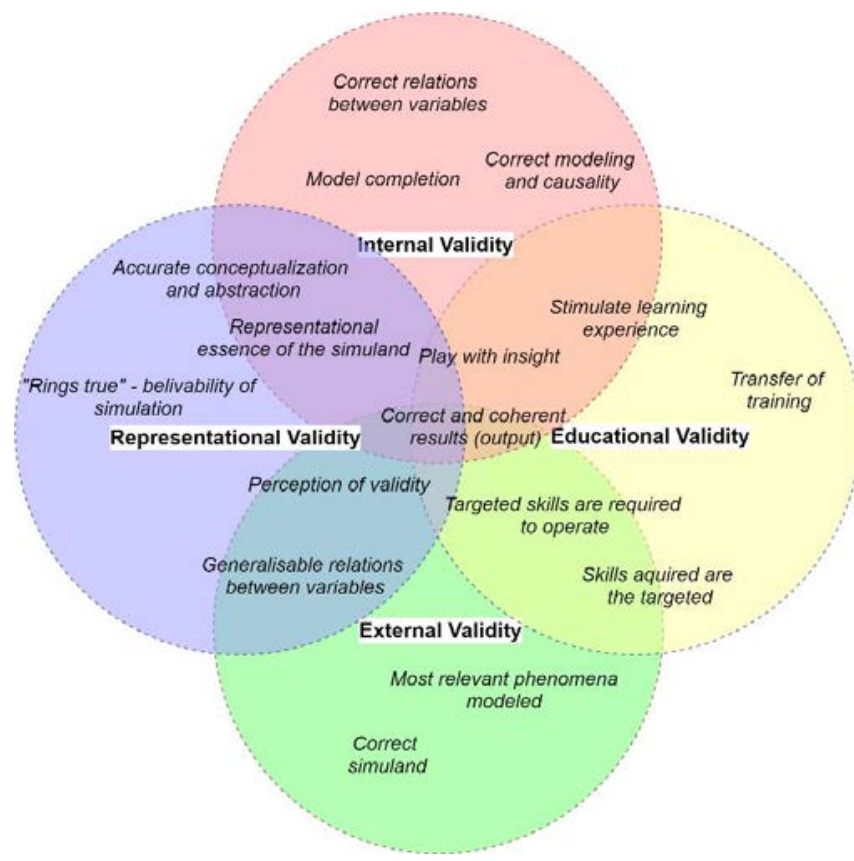

Figure 3: Map of the validity concepts and their relations. Each concept is enrighed with key aspects identified by Feinstein and Cannon (2002)

\section{Representational and Educational Validity}

Representational validity asks the question: does the simulation provide a valid representation of the simuland? This form of validity is of utmost concern in the design and development phases of a simulator as it assesses whether the representations used in a simulation manage to capture the simuland's "essence". This is not the same as (physical) fidelity, which describes perceived realism of the simulation; a representationally valid simulation does not have to appear real or homomorphic (Dahlstrom et al., 2009). The concept entails capturing the theoretical essence of the phenomena being modeled, as opposed to the literal one. Feinstein and Cannon (2002) use the analogy of how art can capture the essence of reality more accurately than a photograph. They argue that the painter both captures and emphasizes key aspects of reality, whereas a photograph replicates the scene with little or no conscious interpretation. Surely, many photographers would frown at this analogy, but I believe it demonstrates the main point of representational validity. For simulations, the artistic interpretation involves conceptualizing and designing correct and insightful abstractions that "ring true" to the simuland.

Educational validity concerns the application value of the simulation as a training technique - whether it serves its purpose as a simulator for training. The concept embodies whether the simulation stimulates learning, provides a valid learning experience, and generates positive transfer of training which will be covered in section 2.1.4. This form of validity can be separated from the other three variants as it evaluates the effect the simulation has on the participating users, rather than the structure and function of the simulation itself. 


\section{Internal and External Validity}

Internal validity basically addresses the extent to which a simulation functions as intended (Feinstein \& Cannon, 2001, 2002). Internal validity can be divided into two parts, where the first part refers to whether the correct things are simulated in a correct manner. This includes whether accurate abstractions and simplifications have been made; whether the simuland is accurately represented; and if elements and variables in the simulations interact and relate to each other so that coherent results (e.g., patterns of output) are generated. This first part of internal validity overlaps with aspects of representational validity. The second part of internal validity reflects how participants and users understand, interact with, and gain insight from the simulation - which relates to external and educational validity. However, the focus is on the participant's perception and understanding of the simulation (e.g., ability to select appropriate strategies to solve simulated task), not whether any learning occurs. According to Feinstein and Cannon, having internal validity does not guarantee educational validity.

External validity reflects the connection between the simulation and the real-world phenomena (Feinstein \& Cannon, 2001, 2002). The main question when assessing external validity is which key components of the simuland should be selected for simulation and whether those components are actually simulated. According to Feinstein and Cannon, the concept of external validity is an area of disagreement among simulation researchers, which is not surprising given the domain-specificity of external validity and the breadth of simulation research. For training simulations, external validity relates to educational validity, as skills taught in the simulation should be transferable to the real world. Similarly, external validity is also the degree to which skills and knowledge used in real-life tasks are necessary for high performance in the corresponding simulated tasks. However, external validity differs from educational validity, as the latter does not imply replication of what would happen in real-life, only that the simulator teaches skills needed for the real-life task. Furthermore, an externally valid training simulator activates the desired skills, but it does not necessarily provide any training effects.

Below, Figure 4 places Feinstein and Cannon's (2002) four validity concepts, together with the descriptive attributes of simulation, in context of the main simulation components that were presented in section 2.1.2.3. 


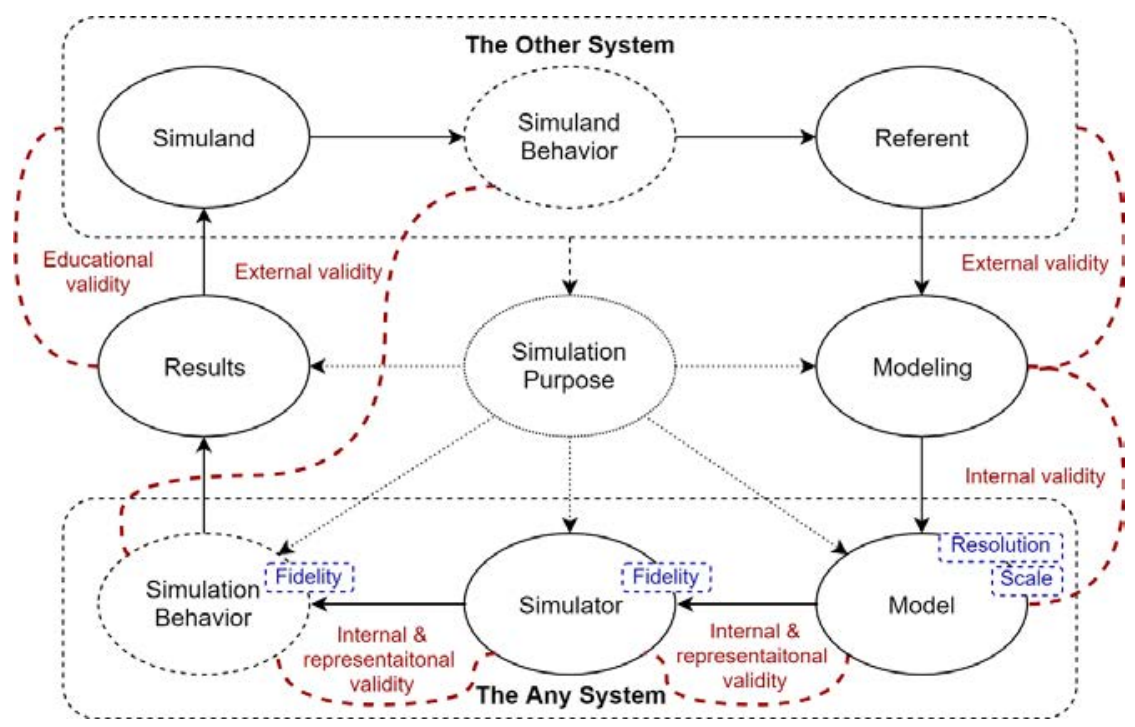

Figure 4: Simulation concepts combined with validity concepts and descriptive attributes.

\subsubsection{Related Constructs}

Beyond the constructs discussed in the sections above, there are three other constructs of importance for this thesis. These are reliability, tractability, and engagement. Reliability refers to the simulation consistency of multiple simulation executions (Bewley \& O’Neil, 2013; McDougall, 2007). According to Bewley and O'Neill, to be reliable, a simulation must produce consistent results (outputs) at different times, between different raters, and between different but equal tasks (e.g., different scenarios in the same domain). For any human-centered simulation, perfect consistency is not feasible because people are not perfectly consistent performers (Bewley \& O'Neil, 2013). Related to reliability is tractability, which is used by Gray (2002) to refer to the difficulty of controlling and managing a simulation. A tractable simulation is - in the context of its purpose - easy to set up, execute, and analyze. In the case of training simulations, it also refers to the amount of training a participant needs to partake in the simulation. Engagement is used by Gray (2002) to describe the degree to which a participant takes the simulation seriously, or in other words, the degree to which a participant can suspend their disbelief. Engagement relates to other terms used in the simulation literature, such as motivation and immersion (Witmer \& Singer, 1998). For a training simulation, engagement is crucial and relates to many aspects of validity.

\subsubsection{Simulation for Training}

As this thesis uses a training simulation case to analyze human-centered simulation constructs, we must look a bit more closely at what training simulations are and how they are used. Simulation for training is one of the five general purposes ${ }^{16}$ for using simulations highlighted by Banks (2010). Studies have shown that simulations are popular and effective techniques for training and assessment of individuals and teams (Daniels \& Auguste, 2013; Maran \& Glavin, 2003; Weaver, Dy, \& Rosen, 2014; Wolfe \& Crookall, 1998). This is because they offer controlled, safe, affordable, and available environments in

\footnotetext{
${ }^{16}$ The other four purposes are analysis, experimentation, engineering, and acquisition
} 
which task-oriented, social, and cognitive skills can be repeatedly engaged, practiced, assessed, and explored (C. M. Banks, 2010; Ören, 2011a; Salas, Cooke, \& Rosen, 2008). Computer-based simulation also has the advantage that the data from the simulations is available directly after the exercise for debriefing and after-action-review (Okuda \& Bryson, 2009; Sawyer \& Deering, 2013). Simulationbased-training (SBT) and simulation-based-team-training (SBTT) (Eppich, Howard, Vozenilek, \& Curran, 2011) follow the experiential learning paradigm, which claims that knowledge and skill are constructed through experience (Kolb, 1984). Simulation enables a form of on-demand "learning by doing" in which skills can be practiced in a situation similar to reality (Fanning \& Gaba, 2007; Maran \& Glavin, 2003; Wolfe \& Crookall, 1998). Most important for SBT simulators is that they actually manage to engage the desired skills and knowledge that are to be trained or assessed (Bewley \& O'Neil, 2013; Feinstein \& Cannon, 2002; Maran \& Glavin, 2003; Van de Walle, Turoff, \& Hiltz, 2010). Incorrect training can have negative consequences - by, for instance, teaching skills that do not apply in real-life (Drews \& Bakdash, 2013; Maran \& Glavin, 2003).

\subsubsection{Types of Users in Training Simulation}

In many constructive and virtual simulations the only user to consider is the one interacting with or interpreting the results of the simulation (e.g., drivers in driving simulations). However, in SBT there are different types of users of the simulator that is important to distinguish. The most obvious user type is the end-user: the participants (or learners), which are those participating in the simulation to learn or have their performance assessed (Lopreiato et al., 2016; Meakim et al., 2013). Another central user is the teacher (or instructor). The teachers are those who use or direct the simulation and determine actions and activities to induce learning for the participants (Candela, 2012). A third type of user, confederates (or embedded participants), are domain professionals (e.g., physicians or nurses) who partake in the simulation in a role that enables and guides the scenario (Meakim et al., 2013). Their role may be to influence the participants in a positive, negative, neutral, or a distracting manner depending on the scenario objectives. Confederates in this sense provide professional realism and challenge to the trainees, which has been recognized to contribute to the psychological fidelity of the simulation (Chang et al., 2015). A common category of confederates used in medical simulations are simulated or standardized patients, which are individuals who have been trained to portray real patients or actual patients trained to present their medical condition in a standardized way (Barrows, 1993).

\subsubsection{Types of Skills Trained}

Skills that can be acquired or enhanced in simulation are often divided into procedural and non-procedural skills $s^{17}$ (often referred to as cognitive skills). Procedural skills are task-oriented and related to knowledge on how (and when) to perform the procedures needed to accomplish a given task (Hardy \& Parasuraman, 1997; Hochmitz \& Yuviler-Gavish, 2011; Salas, Cooke, et al., 2008; Salas, Rosen, Held, \& Weissmuller, 2008). Annett phrased procedural knowledge simply as "knowing how to do things" (Annett, 1996). In the medical domain, procedural skills include skills like performing anesthesia, surgery (e.g., suturing), treatment (e.g., intubation), patient triage, and countless others. These skills are generally developed as a result of practice (Hochmitz \& Yuviler-Gavish, 2011). Surgeons, for instance, acquire procedural skills primarily through "training by doing" (Kneebone, 2003); hence, it is straightforward to see the value of SBT for developing procedural skills (Maran \& Glavin, 2003; Nestel,

${ }^{17}$ Drews and Bakdash (2013) use the terms technical and non-technical skill 
Groom, Eikeland-Husebø, \& O’Donnell, 2011). However, simulation training is often too focused on these skills, downplaying the importance of non-procedural skills and the training environment (Kneebone \& Baillie, 2008). Non-procedural skills are not directly associated with the specific tasks and are often cognitive in nature, such as self-management, decision making, communication, teamwork, leadership and management, situational awareness, and so on. Siu and colleagues (2016) demonstrated that a significant number of avoidable incidents occur in surgery that can be linked to failures in non-procedural skills.

\subsubsection{Transfer of Training and Fidelity}

The intended result of training simulations and training is positive transfer of training. Transfer refers to the application of skills and abilities learned in one setting (training) to another (real world) with the goal of improving performance in the other setting (Salas et al., 1998). Transfer is frequently divided into three categories; positive transfer, in which improved performance is observed in the real world; zero transfer, where no performance change is observed after training; and negative transfer, in which performance is actually decreased after training (Drews \& Bakdash, 2013). Different theories have been proposed on how transfer can be understood. General transfer and specific transfer are two distinct theories that stand at odds with each other (Drews \& Bakdash, 2013). General transfer claims that the cognitive transfer function is broad and that it generalizes from one task to another - even cognitively unrelated ones. Proponents of the specific transfer theory claim that transfer is narrow and will occur if the training task is almost identical to the target scenario and task. Both of these approaches are problematic, as general transfer is difficult to establish where there is no common cognitive basis between tasks, whereas the requirements for specific transfer are practically impossible to achieve. Morris and colleagues suggest a theory of transfer that falls in between; the appropriate processing transfer theory (1977). This thesis will not focus further on theories of transfer, besides noting that, as pointed out by Drews and Bakdash (2013), for transfer to occur, the training task must support the underlying psychological and cognitive operations employed in performing the target task.

Regarding physical fidelity, Hayes and Singer claimed that it is important to "depart from realism in order to provide the most effective training" (1989, p. 15), as this allows for replaying sequences, highlighting key tasks, planning and controlling complicating factors, etc. (Alessi, 1988; Hancock et al., 2010). In selecting the level of physical fidelity, Salas et al. (1998) claim that only the level of fidelity required to successfully train individuals should be used. High physical fidelity is not only costly, it has also been demonstrated that in some cases the absence of physical realism is more effective for training purposes (Alessi, 1988; Dahlstrom et al., 2009). One reason for this may be that high physical fidelity can hinder learning due to overstimulation and less focus on the core task - especially for novice learners (Alessi, 1988; Feinstein \& Cannon, 2002). In line with this, Smallman and St. John (2005) advise that visual displays for training should be created from a minimalist perspective and should present only the essential material needed. Further, training aids can be used to compensate for perceptual deficiencies, by, for instance, including a "declutter" mode to reduce nonessential information. They also suggest that users may need to be given direct, corrective feedback to aid their learning. 


\subsubsection{The Human in Simulation}

Insofar, the theoretical background has presented technical aspects, classifications, definitions, and core concepts of simulations in general. However, to investigate whether a human-centered simulation is similar enough to the real world (the simuland), to design new simulations, or to discuss attributes and core concepts such simulations requires understanding of the buman part of human-centered simulations. This means understanding of human problem solving and cognition. A large part of research and development related to human-centered simulation relies on traditional and deep-rooted assumptions about what human cognition is and how it should be studied - traditions and assumptions that have been criticized in contemporary cognitive science (Hutchins, 1995a; Perry, 2003). The following sections of the chapter will present one such contemporary view of cognition needed to understand human-centered simulations.

\subsection{Theories of Cognition}

This part of the theoretical background will describe distributed cognition, a contemporary perspective on cognition, and why this perspective is suitable for understanding and studying human-centered simulation. Before introducing distributed cognition, I will give a brief description of what I will refer to as the classical view on cognition and its critique.

\subsubsection{What is Cognition?}

A human, or any other autonomous thinker, can be referred to as a cognitive agent. The thinking activities of such agents can generally be referred to as cognition. Neisser (1967 cited in Perry 2009) provided a definition of what cognition is:

\section{"[...] all of the processes by which the sensory input is transformed, reduced, stored, recovered, and used" (Neisser, 1967 cited in Perry 2009).}

Cognitive science is a interdisciplinary research field devoted to studying and understanding what cognition is, how it works, and how cognitive agents achieve intelligent behavior through cognitive processes. Typical examples of such cognitive processes include learning, remembering, sensing, perceiving, planning, and thinking (Frederick Adams \& Aizawa, 2010). The scientific disciplines that are usually considered parts of cognitive science include psychology, linguistics, neuroscience, computer science, anthropology and philosophy (Bechtel, Abrahamsen, \& Graham, 1999; G. Miller, 2003). Cognitive science is a rather recent field, dating back to the 1950s and 1960s, that evolved as an alternative to experimental psychology, which uses laboratory methods for studying mental operations (Perry, 1997). At the time, experimental psychology was dominated by behaviorism, a view that promoted scientific study of the behavior of individuals, which virtually denied the existence of a mind (Bechtel et al., 1999; Graham, 2017; G. Miller, 2003). Through interdisciplinary communication, behaviorism was gradually dismissed, through the so-called cognitive revolution, by researchers who saw that there is more to human behavior than reinforced responses to environmental stimuli: that is, mental (cognitive, representational, or interpretative) activity. What came out of this revolution was the classical view of cognition. 


\subsubsection{Classical View of Cognition}

According to the classical view of cognition, the mental activities - the cognitive processes - of a cognitive agent are realized through internal symbol-manipulation in the brain, which is the main computational device of the agent (Bechtel et al., 1999; Hollnagel, 2011). In the 1960s and 1970s, the idea that the brain is a symbol-manipulating machine gained popularity, and with this came the Physical Symbol System hypothesis (Newell, Simon, Newel, \& Simon, 1976). The PSS-hypothesis states that physical symbol systems are the core architecture of cognition, and that symbol transformations are both necessary and sufficient for intelligence. Combined with the computational theory of mind (CTM), which roughly states that representations account for the nature of intentional states and that cognitive processes are computational in nature (Horst, 1999; Rescorla, 2017), the computer ${ }^{18}$ became the model for the human mind. Thus, the mind became a computational system and core cognitive processes (e.g., reasoning, decision-making, and problem solving) were seen as computations executed by the mind-computer. On this basis, Alan Newell and Herbert Simon used computers to simulate cognitive processes (G. Miller, 2003).

There were two sides of the mind-computer relationship. One side was to build the computer in the image of the human to create artificial computational devices (agents) that were truly intelligent (AI research). The other - fueled by the rapid advancement and promises of technology and optimistic AI research - was to adopt the computer as the working model for human cognitive processes and cognitive architecture. In this way, designing, building, and experimenting with computational models within AI went hand in hand with psychological experimentation (Thagard, 2014). What could be learned from the model, i.e., results from AI and computer science, could then be used to further describe the thing being modeled, i.e., cognition and the brain. Most research in cognitive science at the time was adopted from experimental psychology, which focused on individuals solving abstract problems ${ }^{19}$ in laboratory settings that were stripped of the context of real-life situations. As symbol manipulations were located solely inside the head, this de-contextualization of cognition was considered unproblematic (Bechtel et al., 1999). Overall, the computer-brain relationship was a powerful idea that promised to create artificially intelligent systems and solve the mysteries of human cognition and the brain in one successful compilation.

\subsubsection{Critique of the Classical View}

A growing movement within cognitive science has criticized the classical view (see e.g. Clark, 1998; Hollan et al., 2000; Hollnagel \& Woods, 2005; Hutchins, 1995a; Kirsh, 2010; Perry, 2003; Suchman, 1997), and many now believe that the classical view of cognition is exhausted ${ }^{20}$. One general criticism states that the cognitive activities of individuals cannot be isolated in a de-contextualized brain; it is so embodied and bound to the physical and social environment that it must be understood in context. Hutchins (1995a) provides an analysis of what he calls "the mistake" made by classical cognition. Here, I will highlight what I think is the crucial point of his analysis: that the classical view was built on a faulty model. According to Hutchins, the computer was never built in the image of the human, nor the brain

\footnotetext{
18 Or more precisely, a Universal Turing Machine (Barker-Plummer, 2016), originally described by Alan Turing (1937)

${ }^{19}$ Often called toy-problems; for instance, solving puzzles and performing mental arithmetic

20 The research field is not by any means abandoned, however
} 
for that matter; rather it was modeled on the symbol-manipulating processes of a socio-cultural cognitive system. What this means is that the original model computer (the model cognitive agent), as described by computer scientist and mathematician Alan Turing (cited in Hutchins, 1995a), was not an isolated human mind. Rather, it was a human operator working with her hands, eyes, and tools (such as pen and paper) within a cultural and social setting to perform cognitive problem solving. Properties such as the body and tools in the environment affect cognition in such a way that they cannot be excluded when analyzing and modeling cognition. The mistake was to attribute all cognitive properties solely to the internal processes of the individual and her brain.

The error can be illustrated using Searle's Chinese Room thought experiment (Searle, 1980). Briefly, the experiment proposes that a computer's ability to understand is similar to that of a non-Chinesespeaking person who sits in a room and receives inputted questions written in Chinese. The person can successfully answer the questions using a set of written rules for how to manipulate the Chinese symbols to output answers. The person clearly does not understand or speak Chinese, but it can be argued that the room speaks Chinese ${ }^{21}$. The mistake of the classical view of cognition was to model the activities of the entire room as activities happening inside the head of the theoretical individual running around in the room (Hutchins, 1995a). In other words, the entire room had been assumed to exist inside the skull of the human. To summarize, the physical and social environment, the body, and tools were lost in translation as the classical view had the (unfortunate) consequence of excluding the world from cognition and cognition from the world.

\subsubsection{Contemporary Views of Cognition}

Looking back at Neisser's definition of cognition, there is nothing restricting these cognitive processes solely within the brain of an individual. According to Perry (2003), any unit that performs intelligent, or thinking, activities can be described as a cognitive entity. Broadening the view, any system that exhibits cognitive properties - a collection of parts that together can be said to think or express intelligence can be described as a cognitive system (often referred to as the systems perspective; Norman, 1994). It is important to clarify what is meant by the system part of this concept. According to the International Council of Systems Engineering (INCOSE), a system is a:

\section{"[...] construct or collection of different elements that together produces results not obtainable by the elements alone. The elements can include people, hardware, software, facilities, policies, documents — all things required to produce system-level qualities, properties, characteristics, functions, behavior, and performance." (cited in C. M.

$$
\text { Banks, 2010, p. 3) }
$$

The term "system" is both broad and flexible; a human is a system, a simulator is a system, team(s) of ambulance medics and the equipment they use make up emergency medical systems, which in turn are parts of a larger emergency response system of society. This is a core idea in many theories of contemporary cognitive science: that the cognitive system can include the body, other agents, and social as well as physical parts of the world. Let us consider the "computer" described by Turing, who was a

\footnotetext{
21 The question of whether the room actually understands Chinese or not, which was the original point of the argument, is debatable For the purpose of this thesis, it is sufficient that the room can successfully complete the cognitive task of answering Chinese questions, but the lonely operator cannot
} 
human operator equipped with pen and paper (if we want to bring her up to date we can give her a smartphone instead) that performed cognitive tasks within a work environment. The human operator is a cognitive agent, and as we have seen, the classical view of cognition draws the line of cognition around the agent's brain. The cognitive systems perspective expands these cognitive boundaries, the cognitive unit of analysis, from agent (brain) to a larger system that also includes the pen, paper, smartphone, the social work setting (Perry, 2003).

The boundary extension is made more or less strong in different theories and views on cognition, not only with respect to how far the boundaries are pushed, but also in regard to what the extension entails. Some perspectives on cognition are content with concluding that tools, the body, the environment, and other external factors support, augment or situate the cognitive agent in such a way that they are necessary to include in the study of cognition (see e.g., Kirsh, 2013; Suchman, 1997; Woolgar \& Suchman, 1989). Other perspectives venture further to claim that components of the cognitive system are so coupled to the agent that cognition is externalized, or distributed, over the system (see e.g., Clark, 2007; Hutchins, 1995a). It can be difficult to grasp the distinction between supporting and extending cognition. Returning to Turing's human computer who is now equipped with a smartphone, the phone can be seen as a cognitive aid or support that helps the agent to solve cognitive tasks - whether it be finding information, communicating, scheduling appointments, or performing arithmetic. From a support perspective, the phone can help the agent to remember a scheduled meeting. From an extension perspective, the cognitive tasks are performed by the entire system - not by the agent using tools. The phone transforms the cognitive task of the agent from the memory task of remembering a meeting into a perceptual task of looking at the calendar and reading that a meeting is scheduled. The contextual coupling thus transforms both the problem and the solution spaces for the agent. It is the cognitive system as a whole that solves the task of remembering to attend the meeting; thus this system can be ascribed a memory function (Hollnagel, 2011). It is important to point out that the system views do not deny the cognitive abilities of the "naked" individual. A working brain without a body or context would still be able to think - to execute cognitive processes - but this would be a different form of thinking.

\subsubsection{Distributed Cognition Perspective}

Distributed cognition is a perspective ${ }^{22}$ on all cognition that was developed by Hutchins and colleagues in the mid to late 80 s. Distributed cognition sees cognition as information processing distributed over a unit of analysis that is larger than the individual cognitive agent (Hutchins, 1995a; Norman, 1994; Perry, 2003; Rogers, 1997). Cognition involves one or multiple agents acting in a physical, social, and cultural environment; cognition in the world as opposed to cognition in the head. The goal of distributed cognition is to understand cognition at the system level rather than at the agent level. For example, in the smartphone example from the previous section the cognitive activity of remembering to attend a meeting was solved by the agent-plus-phone system. Distributed cognition has its roots in Edwin Hutchins' seminal text Cognition in the Wild, in which he claims that cognition is:

\footnotetext{
22 Also referred to as a theoretical framework In this thesis, distributed cognition is used as an analytical framework for studying cognitive activities in human-centered simulation
} 


\section{"[...] computationally realized through the creation, transformation, and propagation of representational states." (1995a, p. 49)}

This characterization is similar to Neiser's definition and the classical view of cognition, as described in section 2.2.1.1. Indeed, Hutchins adheres to a view of cognition that is based in the information processing paradigm (Newell \& Simon, 1972 cited in Perry 2003; Perry, 2003) including symbolmanipulation, with the important distinction that cognition as information manipulation-processes does not take place solely within the brain. These larger units of analysis - the cognitive systems - are viewed as information processing. From this perspective, cognitive activities involve informationprocessing concepts such as how information flows and is coordinated across the system, and how information representations are transformed as it propagates through the system (Hutchins, 1995a; Perry, 2003). The notion of representations is central in distributed cognition. A representation is, from this perspective, a structure that stores knowledge about a domain. This knowledge can be manifested in different ways in the cognitive system, for instance within the individual (in neural pathways) or in the environment (e.g., in artifacts, social structures, and cultural practices). All elements in the cognitive system are considered equally important for problem solving, which is viewed as traversing problem states within a problem space until a desired goal state is reached (Perry, 2003). Problem states are representational states, and moving from one representational state to another can be done by transforming representations and propagating information across different elements in the cognitive system.

Distributed cognition follows the functionalistic doctrine, as a cognitive system is characterized by its functions: what it does rather than what it is (Perry, 2003). This is not to say that the physical implementation of the system's elements is unimportant, but the functions that the elements mediate are multiply realizable - meaning that equal functionality can be achieved using different physical structures and representations. For instance, many different handheld devices, calendar applications, or physical calendars can achieve the "remember-meeting-function" in the phone-calendar example above.

\subsubsection{Core Principles of Distribution}

Hollan and colleagues (2000) formulated two governing principles of distributed cognitive systems. The first principle follows the functionalistic doctrine, claiming that relations between elements of the cognitive system are functional rather than physical, which makes the boundary of the system relative rather than absolute. What is not included within the system boundaries becomes the system's environment, which provides the system with inputs and reacts to outputs from the system (Hollnagel \& Woods, 2005). Often there is no clear answer as to where to set the boundary; the question is empirical and relative in regard to where information flows and is transformed (Forsblad, 2016).

The second principle states that information processing occurs not only in the head, but is also distributed in the world. There are three general types of distribution of cognition: across internal and external structures, across social structures, and across time (Hollan et al., 2000), each of which will be presented below. In addition, Furniss and Blandford (2006) have compiled a comprehensive list of distributed cognition principles based on a review of the distributed cognition literature, see Table 4. 


\section{Distribution over Internal and External Structures}

Perhaps the clearest case of distribution is distribution over internal and external structures. Artifacts, or tools, involved in completing the cognitive activity are parts of the cognitive system, rather than sources of external stimuli. These tools do more than aid cognition (e.g., by serving as external memory), rather they transform the cognitive task itself for the agent (Hutchins, 1995b; Kirsh, 1995; Norman, 1994; Perry, 2003; Zhang \& Norman, 1994). To give another example than the phone calendar in section 2.2.1.3; when one is to find outliers in a numerical data set, a graph visualization transforms the task from a mathematical and arithmetical task (thinking) to a perceptual task (seeing). Hollan and colleagues (2000) relate this internal-external coupling to the way in which a blind person's cane or a cell biologist's microscope are central parts of how they perceive, interact with, and think about the world.

A foundational question is why individuals pair with their environments - in other words, how can computational costs be lower when exploiting external structures? Distributed cognition answers this question by describing how people use their environments, from a cost-perspective, in efficient ways. Kirsh (2010), for example, describes the basics of internal-external coupling in terms of computational costs. In problem solving, humans can use internal processing or use external structures for external processing, and will normally use the structure where the total cost is the lowest. For example, Kirsh (1995) demonstrated how individuals arrange the space in their environments to simplify cognitive activity. Spatial arrangements can both highlight (or cue) and constrain key parts of the task at hand to, for instance, reduce perceived actions and decision-points. Ingredients of a dinner can be spatially arranged on the kitchen counter in the order they are to be cut and prepared for the dish. In doing so, spatial arrangements will shape and reduce the internal computational complexity of the cooking activity - thus reducing the cognitive cost. Another example is the way in which Tetris players can offload mental rotation tasks using physical structure - by rotating the Tetris blocks in the screen rather than mentally (Kirsh \& Maglio, 1994). Furthermore, spatial arrangement can simplify perception by clustering and categorization of objects. This is a common strategy when solving a jigsaw puzzle; categorizing pieces according to color or shape reduces visual clutter that is otherwise too complex to process. These are all examples of how the physical environment transforms internal computation through externalization of parts of the computation.

\section{Distribution across Social Structures}

The second type of distributed cognition is across social structures. This means that the social organization itself forms a cognitive architecture, which determines information trajectories and transformations (Hollan et al., 2000). Perhaps the clearest example of this is the way in which cognitive processes can be socially distributed across members of a group, or team, who solve a shared problem (Hutchins, 1995a). In shared problem solving, the task must be decomposed and organized so that each agent can apply their individual expertise and take a specific role in the pursuit of their team's objective (Perry, 2003). According to Perry, this division of labor determines the cognitive architecture of the problemsolving system. To complete the objective, members need to have a communication structure, which will propagate information across the system. In addition, informal communication will propagate information, even if the communication is not explicitly related to the task at hand (Furniss \& 
Blandford, 2006). Beyond communication, information and action can also be coordinated among group members through cues and behavioral triggers.

\section{Distribution over Time}

The third type, distribution of cognition across time, refers to the way that past events or ongoing processes can shape future cognitive states and processes (Hollan et al., 2000). This can be seen in both shorter and longer time frames. In shorter time frames, arrangement of artifacts and space can serve as a function (a cue) for the system to perform a particular task at a later time (Bang \& Timpka, 2003); a classical example is to tie a piece of string around a finger. The artifacts themselves can also provide cues over time about how they are to be used, for instance via wear and tear. Furthermore, Rajkomar and colleagues (2013) showed that time (like external structures) can be used to reduce complexity in cognitive work during short-term activity, for instance by reducing peak complexity and cognitive load by distributing tasks over time. The concept of internalization is an example of distribution across time. Internalization is the process where individuals internalize parts of cognitive processes that were originally distributed in the environment.

In longer time-frames, cultural practices are developed that structure many of our cognitive activities. The history of artifacts and social practices form norms, rules, and knowledge that shape distributed cognitive processes (Hollan et al., 2000). Hutchins (1995a) refers to how ants change their environment using chemical trails that will guide the paths of other ants. Similarly, cultural heritage guides and shapes activities of people as they adopt tools, strategies, and lessons developed by previous generations. For instance, the fact that the color red can indicate alarm or critical information is culturally induced.

\subsubsection{The Study of Distributed Cognition}

Applying the distributed cognition perspective means that many cognitive functions, processes, and representations can be studied directly in the world. As many of the representations are physically manifested in artifacts in the distributed system, a researcher can "enter" a cognitive system to study it (Perry, 2003). This possibility is highlighted by Hutchins in the following quote:

\section{"With systems of socially distributed cognition we can step inside the cognitive system, and while some underlying processes (inside people's heads) remain obscured, a great deal of the internal organization and operation of the system is directly observable"}

$$
\text { (Hutchins, 1995a, p. 129). }
$$

Internal mental representations of individuals are treated as "black boxes", as the distributed cognition level of granularity is only concerned with these internal representations' inputs and outputs. In other words, the actions (e.g., manipulation of external representations) of the individuals in the system. Distributed cognition has mostly been employed to study work-settings (non-simulated), such as airplane cockpits (Hutchins, 1995b), air-traffic control (Halverson, 1994), control rooms (Garbis, 2002), infusion administration (Rajkomar \& Blandford, 2012), patient records (Bang \& Timpka, 2003), collaboration in healthcare (T. Cohen, Blatter, Almeida, Shortliffe, \& Patel, 2006), etc. It has also been used to study other settings, for instance cognitive activities in everyday life (Forsblad, 2016).

Distributed cognition is not a methodology in itself, and several different approaches to studying distributed cognition have been used. The most common approach to gathering data is through 
ethnography (see section 4.1). A distributed cognition analysis of a cognitive system can be made at different levels of granularity, and often a coarse analysis is first needed to provide context to later analyze detailed interactions and cognitive processes. However, few "off-the-shelf" methods operationalize theoretical concepts from distributed cognition and guide the researcher (T. Cohen et al., 2006; Furniss \& Blandford, 2006; Halverson, 2002; Rogers, 1997; Sellberg \& Lindblom, 2014). Hutchins (1995a) suggests a framework based on Marr's (Marr, 1982) three-layered description of how perception is computed. In this "three-level analytical framework" 23 the first level describes the goal and history of the system, the second level describes how representations are propagated and transformed across the system, and the third level describes how the representations are realized by the system elements. However, this framework provides little guidance and structure to realizing such an analysis.

One methodological framework that provides support for distributed cognition analysis is distributed cognition for teamwork (Blandford \& Furniss, 2006; Furniss, 2008; Furniss \& Blandford, 2006, 2010) In short, DiCoT is an attempt to structure and guide distributed cognition studies using a step-by-step procedure and a set of analytical principles (see Table 4). Mostly, DiCoT has been used to study cognitive processes in health care settings (see e.g., Blandford et al., 2015; Furniss, Masci, Curzon, Mayer, \& Blandford, 2015; Rajkomar \& Blandford, 2012; Werth \& Furniss, 2012). Despite its name, DiCoT can be used to analyze individual work as well, as most of its principles adhere to distributed cognition in general. Furthermore, the DiCoT approach is not only intended for analysis. Inspired by contextual design (Beyer \& Holtzblatt, 1998), DiCoT can also be used for (re)design of cognitive systems. Section 4.2 will further describe the DiCoT methodology and how it was used in this thesis to analyze distributed cognition in simulation and to guide design of a simulator.

\subsubsection{Distributed Cognition and Simulations}

In computer science and human-computer-interaction (HCI) research, early information processingbased theories of classical cognition and psychology (for instance the GOMS model) were employed (and are still used) as the cognitive model of how individuals interact with computers (Carroll, 2013; Jacko, 2012). Based on the literature, such classical views of cognition and interaction seem to be the de-facto framework of human-centered simulations in the simulation field, and to the best of my knowledge there are no studies that challenge the classical view in the simulation literature.

However, there are studies within the cognitive science field that use simulated environments to evaluate and discuss distributed cognition processes (see e.g., Chandrasekharan \& Nersessian, 2015; Gillen, Ferguson, Peachey, \& Twining, 2012; Jones, McNeese, Connors, Jefferson, \& Hall, 2004; Smart $\&$ Sycara, 2015). However, as with the majority of research using simulation, the results do not generally contribute to theories and practices of simulation, but rather to what is studied using simulation (i.e., theories of cognition, the general case domain, or the specific case). For instance, Campbell et al. (2008) developed a computerized simulation environment to facilitate emergency response planning and training where they used distributed cognition to study emergency response processes. Another example is Woods and Roth (1995) who discuss simulating distributed cognitive systems using constructive computer simulations. Such simulations of cognition were a central part of the traditional

\footnotetext{
${ }^{23}$ First named so in (Garbis, 2002)
} 
view of cognition, as simulations of cognition were seen as formal theories of human cognition (see e.g., Newell et al., 1976).

There are examples of studies that utilize distributed cognition theory for simulator design. Toups and colleagues (Toups \& Kerne, 2007; Toups, Kerne, Hamilton, \& Blevins, 2009; Toups, Kerne, Hamilton, \& Shahzad, 2011) applied distributed cognition and ethnographic fieldwork to guide design of a digital training simulator for team coordination in fire emergency response. They focused low-level fidelity, what they refer to as the zero-fidelity approach, to highlight human-centered aspects of the work practice in a simulated environment. In short, zero-fidelity simulation design intends to understand and resituate team coordination activities (such as information flow) in an alternative context. Toups and colleagues discuss the fact that real fire emergency response involves quick decisions under stress, which they argue is therefore an important factor to recreate in simulation. However, they derive no implications from using distributed cognition for the field of simulation. 
Chapter 2 


\section{CHAPTER 3 \\ EMERGENCY MEDICAL \\ MANAGEMENT DOMAIN}

This chapter describes the application domain of the thesis, which is management of medical emergency response and especially training in this skillset. Medical response and management of medical response is geographically distributed: it takes place at the site of an accident, en route to accident sites or care locations, at care locations (e.g., a hospital's emergency department), and at backends (e.g., command centers). Together these activities make up an emergency medical service (EMS) system. An EMS system is defined as a system that:

\section{"[...] provides for the arrangement of personnel, facilities, and equipment for the effective and coordinated delivery of health care services under emergency conditions."}

(Moore, 1999)

Appropriate and effective management is crucial for the delivery of health care services (Lennquist, 2009). Essential components of efficient management involve cognitive abilities such as understanding the situation, planning actions, decision-making, and coordinating resources (Blackwell \& Kaufman, 2002; Hick, Barbera, \& Kelen, 2000). Effective management during so-called major incidents (MIs), which are adverse events (or combinations of multiple events) where the everyday response is insufficient, is of critical importance to minimize adverse patient outcomes (Lennquist, 2009). MIs are rare and reallife experience is difficult to accumulate, thus proper training and preparation are critical. The following sections will describe emergency medical management in response to major incidents in a Swedish context, the use of simulation for emergency medical training in general, and the Emergo Train System (ETS) in particular.

\subsection{Major Incidents and Surge Capacity}

In Sweden, management and medical response are structured on local, regional, and national levels (Nilsson, 2013). Local management coordinates resources at the scene of the incident and the individual hospital's response, whereas regional management coordinates resources and distributes patients on a regional level across multiple hospitals over a larger, county wide, geographical area (Lennquist, 2009). National management is concerned with coordinating responses to large cross-county threats (e.g., the forest fire in Västmanland in 2014). The resources and routines of local response are sufficient to administer appropriate care in everyday accidents and incidents that involve a small number of injured people. During MIs, however, these local everyday response resources are inadequate and regional coordination is needed (D. Cohen et al., 2013; Lennquist, 2009; Nilsson, 2013). In such situations, effective management is essential to minimize negative patient outcomes (Blackwell \& Kaufman, 2002). 
At the regional level of management, decisions are made regarding distribution of patients and resources (Lennquist, 2009; Nilsson, 2013). In an MI with multiple severely injured patients, regional management must determine to which care facility each patient is to be sent. This is referred to as distribution keys (patient referrals). In Sweden, this task is initially performed by the designated duty officer (DDO), who has the mandate to declare MIs and initiate regional disaster management. The DDO is thus an important link between everyday health care and preparedness for disasters. The DDO is also the one that confirms a suspected MI based on information from the emergency dispatch center (EDC), on-site reports from the ambulance crew, and information from other agencies. When the DDO confirms that an MI has occurred, the MI is declared and regional hospitals are notified. When an MI has been declared, there is an immediate need for the health care system to increase the medical capacity ${ }^{24}$ to coordinate, treat, and logistically manage a potentially high influx of victims in need of care. This increase in capacity to manage resources and patient flow is generally referred to as surge capacity (Barbisch \& Koenig, 2006). In other words, surge capacity refers to the maximum number of individuals in need of care that the health care system can take care of on sudden demand (Stratton \& Tyler, 2006).

\subsection{Pre-Hospital and In-Hospital Management}

Effective prehospital medical response to major incidents is necessary to adequately care for the injured (Nilsson, 2013). The prehospital response serves many important functions during MIs and is mainly a task for the ambulance service, which often arrives as the first medical resource on-site (Rüter, Nilsson, \& Wikström, 2004). Paramedics arrive in teams of two, and the paramedics in the first ambulance to arrive are tasked with establishing the on-site medical management. In doing so, they take the roles of ambulance incident commander (AIC), who is formally in charge of the medical response at the scene, and medical incident commander (MIC), who has the medical responsibility. The goal of the AIC role is to create the best possible conditions for the medical staff to work on site, which involves communication with regional management, safety-assessment, reporting on the medical need, reporting a possible MI, requesting additional medical resources, and serving as a liaison with the rescue services and the police. The MIC role manages the medical response on site and is responsible for everything that relates to the injured patient or patients. This involves making decisions regarding strategy for triage, treatment and stabilization, and transport of the injured to a care location - as well as ensuring that these tasks are carried out. Sometimes staff roles can be assigned to support the AIC or MIC. All additional medical staff on site take the role of caregivers ${ }^{25}$ that administer triage and treatments and report the medical situation to the MIC.

An important task for prehospital medicine and medical management is triage, which is a process of prioritizing patients according to their need for urgent care (Lennquist, 2009; Nilsson, 2013). The purpose of triage is to establish treatment and transportation priorities. Primary triage is done at the incident site based on examination of patients physiological parameters, such as motor response, respiratory (e.g., breathing rate), and circulatory parameters (e.g., pulse, blood pressure). The priority level is set according to the severity of injury, treatment priority, and transportation ability. There is no

\footnotetext{
${ }^{24}$ Medical capacity is the term used to describe the number of patients that can be attended to within the health care system at any given time

${ }^{25}$ At minor accidents, the MIC and AIC also function as caregivers
} 
single best method for identifying the critically injured, which has resulted in multiple systems for triage (e.g., START and Sieve), which often employ mnemonics for which parameters to assess and in which order. In Sweden, there is no national triage system but many counties adopt a physiological triage protocol. The identified priority level is marked using priority tags, usually bracelets or neckbands, colored red (immediate), yellow (can wait), and green (should wait) ${ }^{26}$. These priority tags are included in the medical team's standard equipment. A secondary triage process is usually performed at a rendezvous location in the vicinity of the accident site.

Treatment and stabilization of injuries is obviously a central aspect of prehospital medicine. An important decision for medical management is to set the level of medical ambition: the extent to which injuries are to be treated at the accident site (Lennquist, 2009). Instead of treating patients at the accident site, one option would be to only administer life-saving care and start organized transport to a care facility as soon as possible. Transporting patients to care facilities is, however, problematic, as time is of the essence and there is a limited capacity for each hospital to receive trauma patients before becoming overwhelmed. Overcrowded emergency departments (EDs) are common in many hospitals, which reduces the surge capacity (Abir, Davis, Sankar, Wong, \& Wang, 2013). Additionally, some patient may require specialized care that is only available at certain hospitals. Therefore, rapid triage must be performed and communicated to regional management so that distribution keys (patient referrals) can be established (Nilsson, 2013).

At the hospitals, nurses at the ED often assume responsibility for the management of emergency response by coordinating the health care activities and caregivers during an MI (Gebbie \& Qureshi, 2002). In the Swedish healthcare system, the head emergency nurse receives the initial MI alert at the $\mathrm{ED}$ and initiates the hospital response according to a disaster response plan (Lennquist, 2009). When patients arrive at the hospital, an additional triage process is used to establish the order in which the patients are to receive care (Lennquist, 2012). According to (Kaji, Koenig, \& Lewis, 2007), effective triage of incoming patients is key to a successful hospital response to MIs.

Management of emergency medicine involves many cognitive tasks that quickly become complicated during major incidents with a high number of patients in need of medical care. Triage is a key cognitive task that is made up of several component tasks (Lennquist, 2009; Nilsson, 2013). Triage consists of multiple related tasks in addition to the task of deciding upon the level of triage for each patient. These include keeping track of the number of patients in need of care and transport, which patients have received triage, which priorities has been assigned, interpreting patient states, what type of care is required, location of the patients, and more. Many of these activities are related to information gathering to construct awareness of the emergency state, which must be propagated in the system and to the environment (e.g., to emergency dispatch or other agencies). As time is a factor and the stakes are high, these tasks must be performed as quickly and efficiently as possible without sacrificing thoroughness.

${ }^{26}$ The colors blue and orange are also used depending on the triage system 


\subsection{Medical Training and Assessment}

The ability of the EMS system to handle MIs effectively depends on appropriate planning and training. Several management problems have been identified in response to MIs, such as late, insufficient or uncontrolled distribution of casualties, flow of information and communication, and inadequate methods for triage (Nilsson, 2013). Many of the management problems have been related to inappropriate training and education for situations where routine knowledge and everyday experience are insufficient (Bolling et al., 2007; Juffermans \& Bierens, 2010). Furthermore, evidence shows that training is essential for effective disaster response management, where a rapid and correct decisionmaking process in the stress and chaos of the moment is of the essence (Auf der Heide, 2006; Brannan, White, \& Bezanson, 2008; Pattillo, 2006).

\subsubsection{Use of Simulations}

Simulation-based training has been growing in popularity for emergency and disaster management to help responders obtain the required knowledge, skills, and experience. Studies have found positive effects from training using simulation methods on various aspects of EMS work, such as team processes, management, patient safety, mass casualty triage, clinical performance, self-efficacy, and more (Brannan et al., 2008; Daniels \& Auguste, 2013; Okuda \& Bryson, 2009; Pattillo, 2006; Salas, Gregory, \& King, 2011; Weaver et al., 2014). Full-scale exercises with a high degree of fidelity are often considered the best approach to teaching medical management (D. Cohen et al., 2013; Gryth et al., 2010). However, there has been criticism of high fidelity training - such as the naïve realism claims and much of medical management training is performed using low-fidelity simulations (Bewley \& O’Neil, 2013; Legemaate, Burkle Jr., \& Bierens, 2012; Lennquist, 2005; Sundnes \& Birnbaum, 2003).

Estimating the impact of training is problematic. A literature review on the efficiency of hospital staff MI training found that there is insufficient evidence to support firm conclusions on the efficiency of any one specific training method (Hsu et al., 2004), and that more evaluation of the efficiency of MI training is needed. This is difficult, as any actual results of MI training can first be seen during a real MI, which are rare, hectic events in which evaluation is difficult. Because of this, simulations are also used for assessment purposes. Increased performance in a simulation setting is commonly used as a measure of training efficiency, based on the assumption that improved performance in the simulated scenario will also translate to the real situation (Grossman \& Salas, 2011).

\subsubsection{The Emergo Train System}

The Emergo Train System ${ }^{\circledR}$ is an analogue, virtual, and low fidelity simulation tool used for teaching emergency medicine and disaster management. ETS is used internationally to train medical professionals in major civil incident doctrines ${ }^{27}$, preparedness, triage, patient management, surge capacity, coordination, and liaison with other agencies (Nilsson, 2013; Nilsson et al., 2013; Rådestad et al., 2012). ETS can be used to simulate an emergency medical scenario, such as an MI, with emphasis on logistics and decision situations, which includes the patients' journeys from the accident site to

\footnotetext{
${ }^{27}$ It is used in the Swedish national concept for prehospital medical command and control at major incidents; visit www.psconcept.se for more information.
} 
hospital admission and subsequently to any specialist departments. Participants in a simulation can include paramedics, hospital staff, regional management, and other agencies. Paramedics can be tasked to identify victims and administer triage, emergency treatments, and transport patients to receiving hospitals. The goal for participants in a simulation session is, as in real-life, to save lives by reducing preventable patient deaths and medical complications. The scale of ETS simulations are adaptable depending on the type of scenario and the purpose of the simulation, as the simulator can be used for anything from small scale training of individual paramedics to large scale surge capacity assessment at a regional (or national) level - which involves multiple organizations and actors. Depending on the scale of the simulated scenario, several instructors may be needed to manage the simulation, which can involve simulation setup, assisting participants, monitoring simulation timers, playing confederate roles (e.g., other actors such as police or fire department), and taking notes for evaluation and performing after-action review.

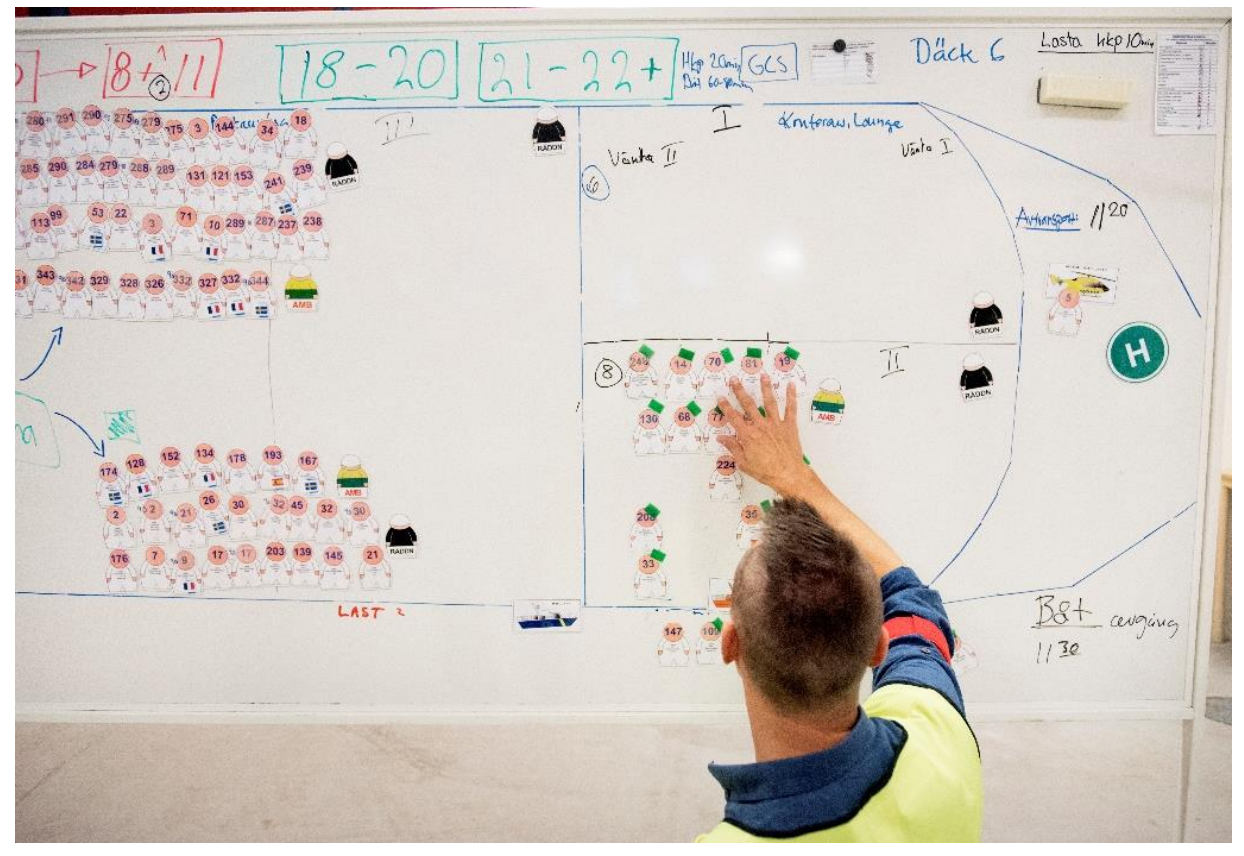

Figure 5: ETS simulation participant engaged in work at an accident site.

As seen in Figure 5, a scenario in ETS is represented on whiteboards using an elaborate set of victim and resource magnet symbols. Typically, one or more whiteboards represent the accident site where the prehospital teams make lifesaving decisions in terms of triage, treatment, and transport of patients - which they do by moving, tagging, and marking the ETS patient and resource figures. A participant in a ETS simulation generally control multiple resource figures. Additional whiteboards can be used to represent the emergency departments, intensive care units, or abstract locations (for instance, ambulances currently in transit). The heart of ETS is its patient bank that contains over 500 patient figures, see Figure 6, all of which have projected outcome measures in terms of preventable death and preventable complications. Triage markers and treatments administered to the patients are indicated by attaching adhesive labels to the figures. The resulting output of an ETS simulation is primarily the 


\section{Chapter 3}

patient outcome, which is measured in whether avoidable complications or deaths have occurred. This is calculated by comparing the time at which a patient reached a care destination or received a treatment with a pre-determined estimate of how much time can pass before appropriate action must be taken for that particular patient.

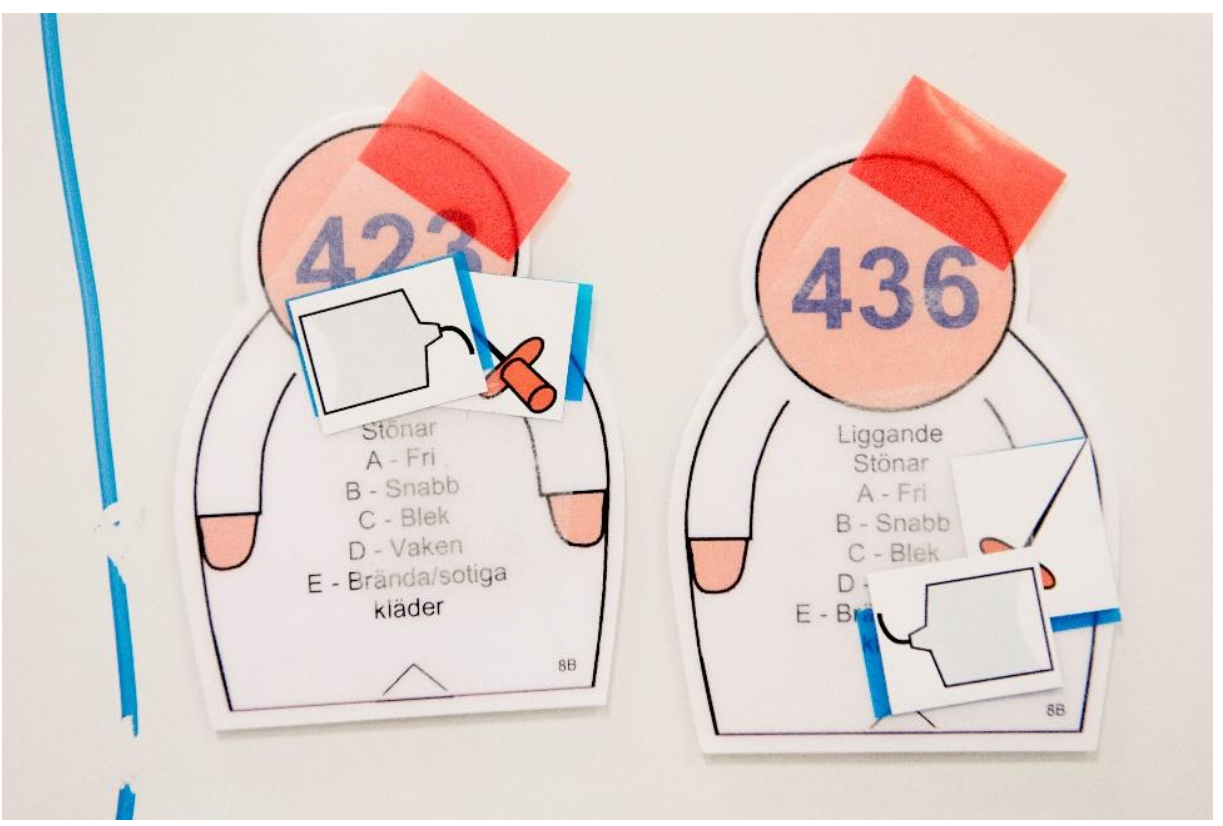

Figure 6: Two ETS magnetic patient figures. Both patients are triaged with a red triage tag and have received IV and fluids treatment, which is represented by adhesive labels. 


\section{CHAPTER 4 RESEARCH APPROACH}

The thesis employs ethnographical methods, design and development methods, and evaluation methods to assess its research questions. Both qualitative and quantitative data collection and analysis approaches were used in the research. To give an overview, Figure 7 shows the studies that have been performed, their relationships, and how they relate to the research papers.

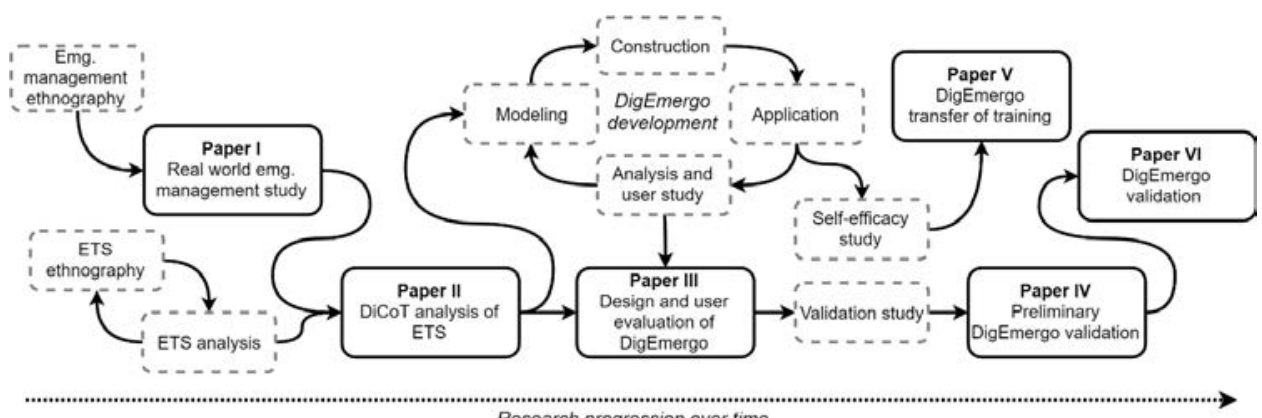

Research progression over time

Figure 7: Published papers and studies. Gray dashed boxes represent studies and black. boxes represent published research papers.

Paper I and Paper II adopted a qualitative ethnographical approach to study, respectively, live emergency management and multiple simulation exercises with the ETS simulator. In Paper II, the DiCoT framework was used for data gathering and analysis procedures. In the following paper, Paper III, the goal was to design a digital variant and re-design of ETS jointly with users of ETS. Following a development structure similar to the M\&S development cycle, the DiCoT analysis was combined with participatory design sessions (Sanders, 2002) and a subsequent iterative prototyping process (Beaudouin-Lafon \& Mackay, 2012). In total, three evaluation studies of the DigEMERGo simulator were performed. The first evaluation was a user study, presented in Paper III. The study combined qualitative and quantitative methods to assess the DigEmerGo prototype's usability and usefulness, as well as users' impressions of the prototype. The goals of Paper IV and Paper V were to assess validity and transfer of training aspects of the DigEMERGo simulator based on controlled experiments, where the resulting data was analyzed using comparative statistics. Paper VI presets additional results from the DigEmergo validation study. The following sections in this chapter will describe the methods and research perspectives that were used, starting with the methods used to study and evaluate the ETS simulator. After that, methods involved in the development and evaluation of the DigEmergo simulator are described. 


\subsection{Ethnographical Methods}

Studying any cognitive system requires an approach to gathering data that can be analyzed. This thesis adopted ethnographic methods to study central information processing activities (e.g., artifact-use, social interaction, use of the environment) during a real MI and in ETS simulations. Ethnography is a qualitative research method, with roots in anthropology and sociology, which is used for studying social phenomena such as people, cultures, activities, etc. via field studies (Agar, 2008). This stands in contrast to the positivistic laboratory-based methods used in classical cognitive science (Perry, 2003). Agar describes ethnography as "a human figuring out what other humans are up to" (Agar, 2008). Further, according to Agar, the goal of ethnography is to give an account of the unit of interest, mainly through observation, participation, and interviews. In the distributed cognition case, this would mean accounting for the cognitive system at work in a particular setting or situation. Agar uses a funnel metaphor to describe the ethnographic research process. Typically, it begins openly with a broad focus, then later narrows and focuses on specific aspects of interest. At the end of the funnel, application of other research methodologies (e.g., controlled experiments or questionnaires) is encouraged to study the phenomenon of interest in detail.

Ethnographic methods are advocated by several design frameworks (Blandford \& Furniss, 2006; Bloomberg \& Burrell, 2012; Perry, 2003), as they can provide understanding of, for instance, system boundaries, the context and environment, tasks, cognitive structures, the user group and the users' needs, and the use of tools. Ethnography has often been adopted for data collection within distributed cognition (Perry, 2003). Hutchins (1995a) describes this version as cognitive ethnography, which focuses on identifying information processing aspects of a cognitive system. Generally, these studies start by identifying (and delimiting) system boundaries, the system's constituent elements, and the goal of the system. In the next stage, data is gathered on system inputs and outputs, information propagation, representation-transformation activities and processes, and transformations leading to the desired goal state. The main method of data gathering in cognitive ethnography is observation, which can be supported by photographs, video capture, interviews, diaries, and questionnaires.

In this thesis, management during a live MI and four typical ETS simulations for teaching and assessing disaster medicine were studied using ethnographic methods, following the funnel metaphor guided by the DiCoT framework. Paper I reports on a full day study of the management activities during the planned response to Göteborgsvarvet, a marathon event with over 60000 participants that was classified in advance as a MI. Data were collected using on-site observations and interviews, as well as NASA task load index (TLX) questionnaires that were handed out hourly to the management personnel. The ETS simulation sessions that were studied, reported on in Paper II, ranged in size and scale from full-day regional surge capacity assessment exercises that engaged multiple organizations, over 20 participants and several instructors, to hour-long local management training sessions with five participating paramedics and a single instructor. Data were collected through participatory and passive observation techniques in combination with interviews. The resulting data from the simulations took the form of personal notes, interview transcripts, and photographs. 


\subsection{Distributed Cognition for Teamwork: DiCoT}

The DiCoT framework codifies ethnographical data using a set of distributed cognition principles (see Table 4) in an attempt to make studies less of a craft and more of an established practice (Blandford \& Furniss, 2006; Furniss \& Blandford, 2006). DiCoT guides the analysis using five themes of distributed cognition as models ${ }^{28}$. Each model contains a sub-set of the DiCoT principles (Furniss, 2008). The first model, the physical layout model, describes physical factors that shape the cognitive system. This includes that which can be physically seen, heard, and accessed in the cognitive space of an agent and, thus, affect (both empower and limit) the computational capabilities of the overall cognitive system. The information flow model describes how information moves and propagates within the cognitive system. This involves identifying what tasks agents are performing, what information they communicate to each other, what their roles are, sequences of actions and events, and so forth. The artifacts and environment model describes how artifacts and tools are used to coordinate and mediate cognitive work. This model focuses on artifacts, representations, and environmental affordances in the analysis by considering their design and their cognitive function in the system. The system evolution model analyzes how a system evolves over time, for instance through reorganization, introduction of new technologies and tools, or by changes in the coordinating abilities of agents (e.g., training). Finally, the social structures model considers how social organization affects coordination of the distributed cognitive system, which can include power relationships between people, political agendas, rapport between individuals, etc.

${ }^{28}$ The last two themes of DiCoT are underdeveloped and difficult to encapsulate within models (Furniss, 2008) 


\section{Physical Layout Model \\ 1 Space and The role of space in supporting cognition for supporting choice and problem solving For Cognition example, Fumiss and Blandford (2006) found that ambulance controllers lay information out on their desks in ways that support their planning See further examples in Kirsch (1995)

\begin{abstract}
2 Perceptual Spatial representations provide more support for cognition than non-spatial ones, provided that Principle there is a clear mapping between the spatial layout of the representation and that which it represents Norman (1994) An example from the ambulance dispatch domain is that calls are displayed on the allocator's screen in order of priority

3 Naturalness Principle

Similarly, cognition is aided when the form of the representation matches the properties of what it represents; in these cases, what is experienced is closer to the actual thing, so the necessary mental transformations for making use of the representation are reduced Norman (1994) This is referred to elsewhere as 'stimulus-response compatibility'
\end{abstract}

4 Subtle Bodily In interacting with the environment, an individual may use their body to support their cognitive Supports processes; for example, pointing at a place in a book while responding to an interruption is part of the mechanism of remembering where we are (Hutchins, 1995a)

5 Situation Awareness

One of the key aspects of shared tasks is that people need to be kept informed of what is going on, what has happened and what is being planned The quality of this situation awareness can be influenced by how accessible the work of the team is This can also be influenced by the proximity of the person, involving both observation and overhearing a conversation See for instance Blandford and Wong (2001) and Hutchins (1995a)

6 Horizon of Observation

The horizon of observation is what can be seen or heard by a person (Hutchins, 1995a) For each person in an environment, this depends on their physical location, the activities they are close to, what they can see, and the manner in which activities take place The horizon of observation of a person plays a large role in influencing their situation awareness

7 Arrangement of From a distributed cognition perspective, the physical layout of equipment affects access to Equipment information and hence the possibilities for computation (Hutchins, 1995a) This applies to the different levels of access to people, their conversations and their work, as well as to physical representations and artifacts For example, in each team, a allocator and radio operator share access to certain items (Furniss \& Blandford, 2006)

\section{Information Flow Model}

8 Information Movement

9 Information Transformation

11 Buffering

10 Information Hubs
Information moves in the cognitive system in various ways, which have different functional consequences for information processing (Hutchins, 1995a) These ways differ in their representation and their physical realization Different mechanisms for information movement include passing physical artifacts, texts, graphic representations, verbal and facial expressions, telephone, e-mail, and alarms

Information can be represented in different forms, and as information moves in the system its representation changes (Hutchins, 1995a) This is referred to as information transformations Appropriate representations support reasoning and problem solving One example of information transformation is filtering, in which information is gathered, sifted and structured In ambulance dispatches, filtering is a central activity of call takers, who solicit and structure information from callers for retransmission to the relevant sector desk (Furniss \& Blandford, 2006)

Information hubs are central focus points where various information channels meet and various information sources are processed together (Blandford \& Wong, 2004) Information hubs can be supported by information buffers, which control the information flow to the hub to keep it working effectively

As information propagates through a system, there may be times when the arrival of new information interferes with important ongoing activity (Hutchins, 1995a) Buffering allows the new information to be held up until the appropriate time for it to be introduced In the case of ambulance control, radio operators frequently buffer information for their sector allocators 
12 Communication Bandwidth

13 Informal Communication

14 Behavioral Trigger Factors
Different communication channels have different bandwidths Face-to-face communication typically imparts more information than computer mediated communication, radio, and telephone (Hutchins, 1995a)

Informal communication can play an important functional role in the system, for instance by propagating important information about the state of the system and the transfer of knowledge through stories, which can have important consequences for learning how the system behaves (Hutchins, 1995a)

It is possible for a group of individuals to operate without knowledge of an overall plan, as each member needs to know only what to do in response to certain local factors These can be dubbed 'trigger factors' because of their property of triggering behavior (Hutchins, 1995a)

\section{Artifacts and Environment Model}

$\begin{array}{ll}\begin{array}{l}15 \text { Mediating } \\ \text { Artifact s }\end{array} & \begin{array}{l}\text { To support activities, people make use of mediating artifacts, which can be any artifacts that } \\ \text { coordinate the completion of a task (Hutchins, 1995a) In ambulance control, the tickets that contain } \\ \text { information about incidents are essential mediating artifacts that are passed around between team } \\ \text { members and annotated to keep track of the state of the incident (Furniss \& Blandford, 2006) }\end{array} \\ \begin{array}{l}\text { 16 Creating } \\ \text { Scaffolding }\end{array} & \begin{array}{l}\text { People constantly use their environment by creating "external scaffolding to simplify their cognitive } \\ \text { tasks" (Hollan et al, 2000, p 192) For example, people often create reminders of where they are in a } \\ \text { task, such as a bookmark }\end{array}\end{array}$

17 Represen- One way that external artifacts can aid cognition is by providing an explicit representation of the tation Goal Parity relationship between the current state and a goal state (Fields \& Wright, 2000; Hutchins, 1995b) The closer the representation is to the cognitive need or goal of the user, the more powerful the representation will be In the case of ambulance control, the allocator's goal is often to obtain a clear screen—one with no outstanding jobs (Furniss \& Blandford, 2006)

18 Coordination Resources are described as abstract information structures that can be internally and externally of Resources coordinated to aid action and cognition by Wright et al (2000) These authors describe six resources that in their Resources Model, which are plans, goals, affordance, history, action-effect, and current state

\section{System Evolution Model}

19 Cultural Heritage

20 Expert
Coupling

Similarly to how ants change their environment using chemical trails, Hutchins (1995a) argues that people (in communities) inherit aa cultural heritage that shape our behavior In Hutchins' case of ship navigation, maps, tools, strategies and lessons developed and laid down by previous generations and adopted by the current crew

Experience improves user performance as users become tightly coupled with their environment, making the computational loops in the cognitive system tighter, faster, and spontaneous (Hollan et al, 2000)

\section{Social Structures Model}

21 Social

Structure and

Goal Structure

Goals can be determined hierarchically and filter down through a hierarchy with overlapping responsibility (Hutchins, 1995a) This can create robustness in the system through group monitoring and job sharing and the system can work through individuals using local goals

22 Socially

Distributed

Properties of

Cognition

The "performance of cognitive tasks that exceed individual abilities is always shaped by a social organisation of distributed cognition" (Hutchins, 1995a, p 262) Two ways that social distribution can be organized: 1) overlap and sharing of responsibilities for enror checking, and 2) separating communication channels to make sure that decisions are robust in checking that multiple independent sources agree

In this thesis, DiCo'T has continually guided the ethnographical data collection of the ETS simulations. After the data gathering was completed, the simulation sessions were analyzed as a single descriptive ETS case study using the first three models of DiCoT - the physical layout model (principles 1-7), the 
information flow model (principles 8-14), and the artifacts and environment model (principles 15-18) - as themes of analysis. Each set of principles was used as a coding scheme in the analysis of each theme, and table-form descriptions and diagrammatic representations of how information propagates through the system were compiled. DiCoT encourages the use of condensed table-form descriptions of the system together with diagrammatic representations of how artifacts and information move around the systems. According to Blandford and Furniss (2006), providing such representations helps to identify strengths and limitations of the current system by highlighting features of the system in relation to the relevant principles. These representations are to be developed iteratively in a process similar to the funnel principle of ethnography. Paper II reports on the ethnographical studies and DiCoT analysis of ETS in detail.

\subsection{M\&S Design and Development Cycle}

Design and development of DigEmergo can be described using the M\&s cycle (C. M. Banks, 2010). The M\&S cycle consists of four major iterative activities: modeling, construction ${ }^{29}$, execution, and analysis. This process emphasizes the cyclical and overlapping aspects of a design and development process (see Figure 8). For instance, findings in the analysis phase can trigger an iteration of remodeling or redesign (construction) ${ }^{30}$. The following sections will describe the M\&S process in relation to development and assessment of the simulator DigEMERGO.

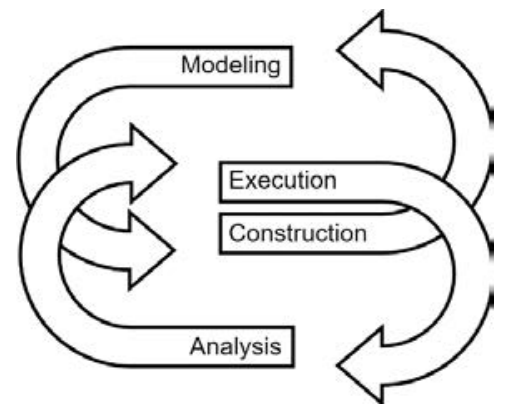

Figure 8: Major phases of the simulation development cycle.

\subsubsection{Modeling}

When developing a simulator, the first steps are (1) to study the simuland, (2) build the referent, (3) construct the conceptual model, and (4) clarify the purpose of the simulation. These actions constitute the modeling phase. The core of the modeling is about simplification, abstraction, and selection of phenomena in the simuland based on the referent. It is in doing this that the conceptual model of the simulation is created. For human-centered simulations, understanding the cognitive (or psychological) factors involved - in context of the simulation purpose - is important for modeling (Drews \& Bakdash, 2013). This includes studying the users, the tasks they are performing, the work contexts, tools used, etc. In this thesis, the ethnographical studies and the DiCoT-analysis of ETS were used to formulate a

\footnotetext{
${ }^{29}$ Construction is referred to as coding in Sokolowski and Banks (Sokolowski \& Banks, 2010) Here, we use the term construction to emphasize that simulations need not be computer based

${ }^{30}$ Evaluation processes should be conducted early and repeatedly throughout design - not only after - so that they can guide development and design (Beaudouin-Lafon \& Mackay, 2012; Jacko, 2012)
} 
claims analysis to identify hypothesized benefits and restrictions of redesigning ETS as a digital simulation system, and to guide the conceptual modeling of DigEMERGo.

In general, distributed cognition theory is considered valuable to the design and evaluation of any HCIsystem (including simulators) as it enables detailed studies of cognitive processes and demonstrates how contextualized human-thinking activities are (pre)arranged (Perry, 2003). Furniss and Blandford (2010) argue that DiCoT contributes to design (modeling in this case) in multiple ways. First, it provides an understanding of the basic mechanics of the system and how it actually works - not just how it should work. This includes understanding a system's strengths and weaknesses, thus seeing what can be improved and what should not be lost in a redesign. Beyond basic mechanics, DiCoT provides a deeper conceptual insight into important elements of the cognitive system in terms of distributed cognition concepts, such as information flow and representation transformations. However, for performing abstractions, simplifications, and selections of simuland phenomena, DiCoT offers no explicit guidance for designing simulations, as it was not specifically intended to be a methodology for simulation design.

As a means of supporting system (re)design, the DiCoT models serve as tools for reflection in considering alternative designs or arrangements of the system (Furniss \& Blandford, 2010). This allows the designer to evaluate the potential strengths and weaknesses of a design. Blandford and Furniss (2006) suggest using claims analysis, a form of "cognitive design rationale" described by Carroll and Rosson (1992), as the vehicle for such considerations. A claims analysis identifies features that strongly influence the system and records the benefits and restrictions (pros and cons) of each feature. Figure 9, which is an excerpt from Furniss and Blandford (2006), shows an example of pros and cons of communication re-designs. In the context of the DiCoT analysis, each identified feature is grounded in distributed cognition theory. Features of a non-cognitive nature, such as costs of set-up or maintenance, are not considered in the claims analysis in DiCoT.

\begin{tabular}{|l|l}
\hline Design Feature & Hypothesized Pros (+) or Cons (-) of the Feature \\
\hline $\begin{array}{l}\text { Have a one-stop } \\
\text { communication channel } \\
\text { with crews }\end{array}$ & $\begin{array}{l}\text { + allows greater flexibility in contacting crew } \\
\text { + could open potential for telephone dispatcher and radio } \\
\text { operator to job share } \\
\text { + can improve communications between crew and } \\
\text { external party }\end{array}$ \\
\hline $\begin{array}{l}\text { Further automate } \\
\text { allocating crews at a } \\
\text { station }\end{array}$ & $\begin{array}{l}\text { + telephone dispatchers call to station is automated } \\
\text { freeing them for other tasks } \\
\text { + allocator can treat allocating a call to a station in much } \\
\text { the same manner as a mobile vehicle } \\
\text { - automation will need further equipment and the addition } \\
\text { of activities performed by the crew } \\
\text { - reducing verbal communication between LAS staff and } \\
\text { crews might have negative social consequences }\end{array}$ \\
\hline
\end{tabular}

Figure 9: Example outtake from a claims analysis in Furniss and Blandford (2006).

\subsubsection{Construction}

When the conceptual model and purpose of the simulation are clear, the simulation technology and the level of required physical fidelity can be explored (Drews \& Bakdash, 2013). In doing so, the creative design process and implementation starts, which will result in a simulator prototype (C. M. Banks, 
2010). There are many frameworks and perspectives for structuring and informing design of techniques in the literature that can be used for human-centered simulation design processes, for example, different types of task analysis (Crandall, Klein, \& Hoffman, 2006; Naikar, Hopcroft, \& Moylan, 2005) from human factors and the many forms of user-centered design from human-computer interaction (Jacko, 2012). This thesis adopts a prototype-based design approach guided by DiCoT. Prototypes are used to inform the design process by generating and articulating design ideas, as well as by collecting feedback and critique from users (Beaudouin-Lafon \& Mackay, 2012). In HCI, a prototype is a physical representation of an interactive system, which can be used to envision and reflect upon the design. Beaudouin-Lafon and Mackay characterize prototypes along four dimensions; representation describes the form (e.g., paper or digital prototype); precision is the prototype's level of detail; interactivity describes the degree to which users can interact with the prototype; and evolution describes the expected lifecycle of the prototype - throwaway or iterative. Successful software prototypes often transform - in a set of iterations - into the final product. Selecting the simulation technology and the level of physical fidelity is a question of objectives, purpose, and costs (Scerbo \& Dawson, 2007), which can be explored using prototypes.

\subsubsection{Execution}

Having constructed a simulator prototype, whether it is paper-based or a precise digital implementation, the next step is to execute the simulation. According to Banks (2010), execution involves setting up the physical system (hardware, networks, etc.) required to run the simulation code. The process of verification can be perceived as part of execution ${ }^{31}$, as verification is concerned with analyzing whether the simulator prototype (the executable model) has been correctly implemented according to its specification and confirming that the hardware is functional. In other words, verification consists of asserting whether the simulation is correctly built according to specification (Feinstein \& Cannon, 2002; Pegden et al., 1995; Sargent, 1996). This can be done using code debugging or technical inspection in which organized teams of developers and testers use their expertise to inspect relevant design documents, algorithms, and programming code (Petty, 2010). The execution step, including verification, is not emphasized in this thesis, as it is of more interest for complex and technologically advanced simulations that require vast computational performance to generate exact numerical outputs.

\subsubsection{Analysis}

Evaluation is a central part of prototyping. The simulation field also has a strong tradition of evaluation, known as the VV\&A process (Verification, Validation, and Accreditation). The latter, Accreditation, is about certifying the simulation for a specific use, which is done by an official authority responsible for that specific domain. I will not consider this issue further in this thesis. Evaluation and analysis activities in the simulation community, and in this thesis, are focused on validation, which is about comparing the simulation with the simuland to determine whether or not the simulation reflects reality ${ }^{32}$ (Rohrer, 2000). Feinstein and Cannon formulated validation as follows:

\footnotetext{
${ }^{31}$ Banks (2010) place verification as part of analysis, which I do not deny, but in my view and for the purpose of the thesis verification is included as part of execution

32 Validation in part overlaps verification Winsberg (2015) argues that the two should not be regarded as two distinct activities
} 


\section{"[...] the process of assessing that the conclusions reached from a simulation are similar to those reached in the real-world system being modeled" (Feinstein \& Cannon, 2002, p. 427).}

For this thesis, validation is concerned both with comparing output of the simulation, in terms of human performance, and with what happens during simulation, viewed as functions and activities of the cognitive system. Just as the concept of validity is diverse and multifaceted, so are the different methods used for validation, and more than one validation method should be used (Petty, 2010). Below, I will describe common validation approaches and the studies performed in this thesis to validate DigEmergo.

\subsubsection{Types of Validation}

Petty (2010) categorized over 90 suggested methods of validation and verification as formal, static, informal, and dynamic approaches. The formal methods rely on detailed quantitative mathematical analysis, such as inductive assertions or predicate calculus (Petty, 2010). Static methods involve technical experts (e.g., simulation researchers, designers, programmers, etc.) assessing validity without executing the simulation (using e.g., analysis of programming code, interface analysis, or cause-effect-graphing). Informal methods are generally qualitative and based on subjective human evaluation. The final category, dynamic methods, assess validity by executing the simulation and evaluating the simulation's outputs, results, or processes. Two of these categories of validation, formal and static, fall outside the scope of this thesis.

One of the most common forms of informal methods is face-validation, which involves qualitatively comparing the simulation results and behavior to the simulands. Potential users or subject-matterexperts (SMEs) observe or participate in the simulation to judge the validity of the simulation based on their knowledge of the simuland. If the simulation differs from the experts' expectations, experience, and knowledge, there might be issues with the conceptual model or simulator design. According to Petty, this approach to validation is arguably the most appropriate for simulations in which "the experience of a user interacting with the simulation is an important part of its application" (2010, p. 342).

Dynamic validation methods are perhaps the most commonly used category of methods in the literature on human-centered simulation. Such validation studies generally include numerical comparisons of simulation data to simuland data (Petty, 2010). Predictive validation is one such dynamic method, in which outcomes of simuland behavior are compared to corresponding outcomes of the simulation. For instance, results of a simulated flight can be compared to logs of a real flight with a plane of the same model. Comparison testing is a form of predictive validation (ibid.), but rather than comparing simulation data to simuland data (for instance, practical reasons might make simuland comparisons impossible), the comparison is made with results from another simulation. The idea is that even if none of the simulations can be assumed to be valid, discrepancies found between the two simulations can reveal validity issues in a shared conceptual model. Another form of predictive validation is to compare human performance of different user groups (often experts and novices) using the same simulation. If one group is assumed to outperform the other in the simuland task, the group should also do so in simulation - which would indicate validity (e.g., if experts outperforms novices). This approach has 
frequently been used to validate surgical training simulations, where the method is referred to as construct validation (see e.g. Gallagher \& Satava, 2002; Mathis \& Wiegmann, 2007).

It is not always the case that an ideal validation method can be selected, for instance due to availability of simuland data, cost, or practicality. When evaluating training simulations, methods for measuring real-life performance before and after training (and over time) are desired to reveal positive transfer and determine educational validity (Carter et al., 2006). However, such longitudinal studies are often impractical and costly in terms of time and resources; thus, alternative approaches such as comparison testing are a common practice for evaluation of SBT and SBTT in healthcare (Weaver et al., 2014).

\subsubsection{Validation Based on Human Performance}

The choice of validation method and which variables to investigate should be bound to the purpose of a simulation. For instance, for training simulations the units of interest are those related to learning and positive transfer of training (see section 2.1.4), which is often measured as changes in human performance. Validating a simulation based on human performance usually requires some measurement of performance, which most often considers the results of an action or set of actions (task-outcomes). However, knowing what to measure is no simple task, as there is no "one size fits all" approach. Furthermore, is not always clear what the important components of the performance are (M. A. Rosen \& Weaver, 2010; Salas, Rosen, et al., 2008). Often performance is viewed as the outcome of an action, but performance can also be defined as the action itself (J. P. Campbell, 1990). From this view, cognitive processes during SBT are a critical aspect of performance. Thus, understanding and analyzing cognition processes, for instance using $\mathrm{DiCoT}$, is a vital aspect of understanding performance.

Whether viewed as a result, presence, or quality of some action, a performance measure is a quantification to rate performance (Bewley \& O'Neil, 2013). There are both qualitative and quantitative methods for measuring performance (Franc, Ingrassia, Verde, Colombo, \& Della Corte, 2014; M. A. Rosen \& Weaver, 2010; Salas, Rosen, et al., 2008). Performance measures can take an objective (e.g., number of tasks completed, accuracy, task completion time) or subjective (e.g., self-reports, quality of work) form. Common methods for measuring performance in simulation include human raters that score performance, self-assessment reports and questionnaires filled in by the participants, and automated scoring conducted by the simulator itself. The latter has the advantage of being non-invasive, precise, and containing few measuring biases. Another way to look at the effect and effectiveness of SBT is to assess performance indirectly by measuring variables related to performance.

\section{Self-Efficacy}

An affective aspect related to performance is self-efficacy, which is the extent to which an individual believes that he or she is capable of performing a specific behavior in a specific situation (Bandura, 1997). This measure has been used previously in healthcare to understand the potential impact of an educational intervention, such as SBT, on clinical practice (Artino et al., 2012; Hsu et al., 2004; M. A. Rosen et al., 2008). Self-efficacy can be divided into specific and general efficacy, where the former is the degree to which the individual believes themselves to be capable of performing a specific task, and the latter is the belief that they can cope successfully with and adapt to difficult situations. Self-efficacy can be increased in training via experience from one's own (or observing others') successful performance 
in a situation similar to reality (Bandura, 1997), or through reducing stress and anxiety, which affects self-efficacy negatively. It has been found that individuals with high self-efficacy find more effective ways to solve problems, and self-efficacy can thus be used as an indicator of performance (Cant \& Cooper, 2010). Moreover, in educational situations, individuals with high self-efficacy tend to learn more and achieve higher performance ratings compared to those with low self-efficacy (Bandura, 1997). The general self-efficacy (GSE) questionnaire is common for measuring self-efficacy. It was developed by Schwarzer and Jerusalem (1995) to "assesses the strength of an individual's belief in his/her own ability to respond to novel or difficult situations and to deal with any associated obstacles or setbacks" (p. 35). In GSE, participants rate ten statements related to self-efficacy on a four point Likert scale.

\section{Workload}

Another indirect measure of performance is workload, which measures how straining or effortful a person considered a work-task or activity to be (Hart \& Staveland, 1988). Hart and Staveland (1988) defined workload as "a hypothetical construct that represents the cost incurred by a human operator to achieve a particular level of performance". Workload is often seen as something that affects performance, as a workload that is too high can lead to decreased performance (Yurko, Scerbo, Prabhu, Acker, \& Stefanidis, 2010). However, high workload does not necessarily imply lower performance. It has been shown that individuals can, through adaptation strategies, maintain a high workload with little or no loss in performance (Sheridan, Wickens, Parasuraman, Sheridan, \& Wickens, 2008). Workload is linked to the level of challenge (the required cost) posed to participants in a simulation, in which low workload is associated with easy tasks and difficult tasks produce higher workload (Prytz et al., 2016; Yurko et al., 2010). Andersson and colleagues (2014) argue that a lack of challenge can be a problem during training, as low challenge does not provide incentive for participants to explore alternative solutions. Relatedly, workload has been used as a measure, or indicator, of transfer of training (see for instance Britt et al., 2015; Scerbo et al., 2013).

The most common method for measuring workload is probably the NASA TLX questionnaire (Hart \& Staveland, 1988), which has been repeatedly validated (Grier, 2015). This questionnaire rates workload on six subscales: mental demands, physical demands, temporal demands, estimated performance, effort, and frustration. Ratings are commonly marked on a line ranging from low to high, where low ratings indicate low workload and high ratings indicate high workload for all subscales except performance, where the scales are reversed.

\subsubsection{DigEmergo Validation Studies}

Evaluation and validation studies of DigEmergo are reported in Paper III, Paper IV, Paper V, and Paper VI. Paper III describes an initial formative user study of the DigEMERGO prototype. This study employed expert users of ETS (certified senior instructors) as participants in prototype simulations, with the intention of investigating and measuring usefulness and ease of use of the prototype. A secondary goal of the study was to guide further development of DigEMERGo by taking advantage of the expert group's rich experience of core ETS features. In this sense, the study was a face-validation study, as the experts judged validity aspects of the simulation based on their knowledge of ETS and the emergency medicine simuland. Usefulness and ease of use of DigEMERGO was measured using the technology acceptance model (TAM) questionnaire (Davis, 1989), contextualized by qualitative results from the simulation sessions (observations and post-simulation interviews) supplemented with a free- 
text questionnaire. The TAM questionnaire consists of twelve statements that are scored on sevengraded Likert scales. As the results were indicative and formative in purpose, no statistical analysis or comparisons were made.

In the second evaluation study of DigEmergo (reported in Paper IV), a comparative construct validation approach (between group expert-novice performance) was adopted to compare surge capacity management performance and workload differences between experts and novices. The expert group consisted of registered nurses, physicians, or medical residents recruited at a local hospital ED. The novice group was made up of university students with no reported medical management experience. In Paper IV, three measures of medical triage were analyzed and presented: time to triage, number of patients triaged, and triage accuracy. In addition, measurements of performance regarding patient treatment and patient disposal (transport) are presented in Paper VI. Furthermore, Paper VI presents results from the NASA TLX questionnaire, which were analyzed in the same manner to explore differences in perceived workload between the two groups. For all performance measures, two-tailed independent sample t-tests were used to compare the two groups, with $\mathrm{p}<0.05$ as the threshold.

A third evaluation study, described in Paper $\mathrm{V}$, investigated aspects of transfer of training through perceived changes in ED nurses' self-efficacy that related to managing a surge capacity event at an ED. Both the second and the third evaluation study considered two categories of patients: in-hospital patients who were already at the ED and trauma patients who arrived from the accident site. Participants took part in three simulation scenarios, where increased self-efficacy post-simulation was considered as positive transfer. For these evaluations, a Swedish translation of the GSE questionnaire (S-GSE; Löve, Moore, \& Hensing, 2012) was used together with a custom questionnaire for measuring task-specific self-efficacy. Moreover, performance related to management skills was measured using simulator-recorded data on the number of patients that received care and the time to treatment. Oneway t-tests with $\mathrm{p}<0.05$ were used to compare the pre- and post-test scores of self-efficacy and to compare management skill differences between the first and the third scenario. The predicted effects were that self-efficacy would be increased in post-test and that management skill would improve from the first to the third scenario. 


\section{CHAPTER 5 \\ APPLYING DISTRIBUTED COGNITION}

This chapter will discuss the results of the research papers. First, the applications of distributed cognition using the DiCoT methodology to study and evaluate cognitive properties of human-centered simulations based on the studies of the ETS training simulator will be discussed. Second, the design and development process of the DigEMERGo simulator and its evaluation will be described.

\subsection{Evaluating ETS Simulations with DiCoT}

The DiCoT analysis of the ETS system, presented in Paper II, revealed that the structure of ETS was flexible and adaptable, both with regard to the purpose of simulation and also during the simulation, through its use of low-fidelity representations and physical artifacts. This flexibility makes it possible to use ETS to simulate different types of scenarios, such as accident sites, hospital settings, or command centers - such as the off-site local medical management of Göteborgsvarvet, studied in Paper I. The patient representations, which are composed of lines of text on magnetic figure cards, facilitate medical decision-making but not medical practice. The open physical layout of artifacts and environment, flexible interaction, and customizable representations allow participants to structure their workspace to generate cues and cognitive scaffolds to manage tasks and coordinate teamwork. Participants frequently use whiteboards as information hubs on which patients are grouped, highlighted, and annotated so that tasks can be coordinated. In doing so, the whiteboards transform the representations of information to make it open and public, which propagates information throughout the cognitive system. In addition, instructors use these properties of the simulator to their advantage by, for instance, using spatial cues to support their situational awareness and as triggers for upcoming simulation events. Core attributes and properties of the ETS simulator that are related to distributed cognition are presented in Table 1 in Paper III.

The established cognitive system and its functions in simulation were also connected to aspects of validity (see Table 3 in Paper II and Table 2 in Paper III). For instance, the open layout and flexible interaction style of ETS were considered to be important and valuable traits for the simulation's representational and educational validity. The ETS artifacts mediated cognition and collaboration in ways that enabled core medical management tasks and contributed to making the simulator understandable, immersive, and easy to use. However, high flexibility of the physical artifacts made it problematic for both participants and instructors to keep track of and control the many variables and processes during simulation, such as patient transports, treatment times, upcoming events, and improvised resources that were not included in the scenario. Managing time-bound processes was a particular cause for concern. For example, participants often "cut corners" to, for instance, start 
treatment of patients without having the correct resources available or to initiate transport of patients before ongoing treatments were finished. Corner cutting here does not refer to intentional "cheating", rather it refers to uncontrolled interaction and non-adherence to waiting times. Such interaction could skew simulation outcome measurements, which are based on the time at which a patient reached a care destination or received a treatment. Nevertheless, these analog simulation mechanics enable a high degree of flexibility and off-the-cuff improvisation.

Another issue identified in the DiCoT analysis was that the openness of the simulation artifacts in combination with participant idle time (due to the abstraction of medical practice) allowed for unintended information propagation within the cognitive system. During such idle time, participants could acquire an unrealistic awareness of the situation, as they were able to explore their horizons of observation. In training simulations, this can serve the purpose of the simulation and its educational validity, as idle time presents additional opportunities for learning and reflection. However, unrealistic situational awareness is problematic when using the simulator as a means of assessing real-life performance capability. Relatedly, since patients in ETS have static medical states that never change there is no need to re-assess medical states during simulation, which could affect external, internal, and possibly educational validity negatively, depending on the purpose of the simulation. However, static representations can benefit the training of novice participants, as it simplifies management tasks. The validity-related issues of ETS that were revealed by the DiCoT analysis are summarized in Table 2 in Paper III. Regarding this, the internal and external validity of the simulation, as well as its applicational validity when used as an assessment technique, were threatened due to unrealistic situational awareness and inconsistencies caused be corner cutting.

The results show that distributed cognition, using the DiCoT methodology, is a promising tool for analyzing and evaluating human-centered simulations. DiCoT can be used to identify distributed computational mechanisms of cognitive systems in simulated environments, which can explain how elements of the simulator enable and mediate cognitive functions and behavior during simulation. This approach allows for understanding of the role of the simulator in cognitive tasks, thereby providing a way to analyze and identify what is important to capture in human-centered simulation when building new simulators. Furthermore, these results demonstrate the applicability of distributed cognition and DiCoT in a novel context and for a novel purpose.

Beyond identification of cognitive processes in simulation, the results show that distributed cognition can be a valuable theory for understanding dimensions of simulation validity. Demonstrating how cognitive processes are bound to the simulation environment and simulator components facilitates assessment of the validity of the simulation. Moreover, by comparing cognitive processes in simulations to their counterparts in reality, simulation validity can be assessed both theoretically and empirically. Such comparisons could be done by analyzing both the simuland and the simulation with DiCoT. In addition, a stand-alone DiCoT-analysis of a simulation can identify cognitive processes that occur as unintended byproducts of the simulator design - processes that do not take place in the simuland.

\subsection{Simulator Development using DiCoT: DigEmergo}

In the previous section, I demonstrated how distributed cognition can be used to study and evaluate human-centered simulations. This section will describe how DiCoT can be used to motivate (re)design 
and guide the development process of human-centered simulators. In doing so, the section will briefly present the DigEmergo system, how DiCoT contributed to its development, and the results of the evaluation studies.

\subsubsection{Modeling}

The study reported in Paper I provided referent knowledge of medical management and the DiCoTanalysis in Paper II provided conceptual insights into how distributed cognitive systems are manifested in ETS simulations. This understanding illustrated the strengths and weaknesses of ETS as a simulator - in other words, what can be improved and what should not be lost in a redesign. The claims analysis (Table 4 in Paper II) found that the arguments for creating a digital redesign were, first and foremost, to increase simulation control to enable accurate simulation outcomes and lower instructor workload. This would make the simulator more tractable (Gray, 2002) for instructors to manage. Furthermore, a digital redesign could also provide a platform that would enable dynamic content (e.g., dynamic patient states, environments, events, integrated media, etc.) to create new opportunities for immersion, use of expert experience, and new types of training and assessment applications. However, the analysis revealed that a digital redesign would run the risk of losing ETS' improvisational opportunities as well as its intuitive interaction mechanisms, which are based on tangible objects (magnetic figures, adhesive notes, pens and paper, etc.) that are easy to use and understand. Moreover, a digital redesign could also make the simulator less tractable, as participants might need additional training to operate it.

The modeling phase of DigEmergo was guided by DiCoT, as it had identified core features and central distributed cognition mechanics of ETS that were important to retain in the digital simulator. Such referent knowledge informs the conceptual modeling and the level of fidelity required to enable the mechanics that are essential for the cognitive system's functionality. Important aspects of ETS to retain were, among others, its style of interaction, which enables face-to-face interaction between participants; its flexibility, which allows for different types of scenarios and improvisation; and the use of confederate and teaching roles for the instructors.

The DiCoT analysis further highlighted how the digital simulator could increase aspects of validity. For instance, the issue concerning corner cutting could be inherently controlled by the digital simulator using a set of implemented rules (i.e., constraints), which could be selected by instructors to best serve the purpose of a specific simulation scenario. Based on the rules, the digital simulator could prevent certain actions for which prerequisite conditions were not fulfilled, such as treating a patient without having the required resources available. In this way, internal, external and educational validity, as well as reliability of the simulation could be increased. Table 4 in Paper III further presents how the DigEMERGO simulator design mitigates validity issues that were identified in ETS.

\subsubsection{Construction}

The design process of DigEMERGO was guided by phrasing ETS core features and validity in terms of distributed cognition, described in Paper III, and a prototyping approach based on participatory design. Using the prototype description of Beaudouin-Lafon and Mackay (2012), DigEMERGO was iteratively developed to become a digital prototype with high precision (level of detail) and full interactivity. The intention, which is beyond the scope of this thesis, is that the DigEMERGo prototype can be further developed into a fully functional and usable final product. 
A key design decision for the DigEmergo system was to replicate the graphical representations and interaction style of ETS, with the intention of retaining familiarity with the ETS system. For instance, triage markers were designed to look like a digital adhesive label (i.e., rectangular shapes attached to digital patient figures) to retain the underlying distributed cognitive principles captured by ETS and enable a degree of familiarity between the digital and analog simulators. Multi-touch screens were used in DigEmergo to allow for direct manipulations of the patient figures in an effort to adopt the ETS interaction style in a digital medium. Furthermore, the screens allow multiple users to interact with DigEMERGo at the same time, which preserves the collaborative work-style of ETS. Direct interaction with the touchscreen also allows for simulated work practice and cognitive scaffolds, such as creating patient groups and informal queues. In this sense, the DiCo'T analysis provided mechanisms for determining the physical fidelity required to establish cognitive functionality. These mechanisms can be useful for both analysis and design of simulators with respect to the physical characteristics that are required to enable the use of cost-effective yet efficient simulations with low physical fidelity. Table 3 in Paper III summarizes how key features of ETS were retained and implemented in DigEMERGO.

The DigEmergo system runs on standard Windows computers using large multi-touch wall screens (i.e., digital whiteboards), see Figure 10. The system is network-based, which makes it possible to connect clients (i.e., user interfaces) from different locations to a shared simulation. Clients can either participate in the simulation (e.g., as different locations such as the accident site or emergency department) or monitor the simulation as observers. A central database on the host computer stores all built-in knowledge and rules of the simulation regarding, for instance, how treatments are allowed to be administered. Such digital features can increase the tractability of the simulator, since it can decrease the difficulty for instructors to control and manage the simulation. Both ETS and DiGEMERGO should be considered as virtual simulators since both use simulated patients and confederate roles. However, ETS should be considered a local simulation that is constrained to the physical location, whereas DigEmergo simulations have no such physical constraints, making it geographically distributable.

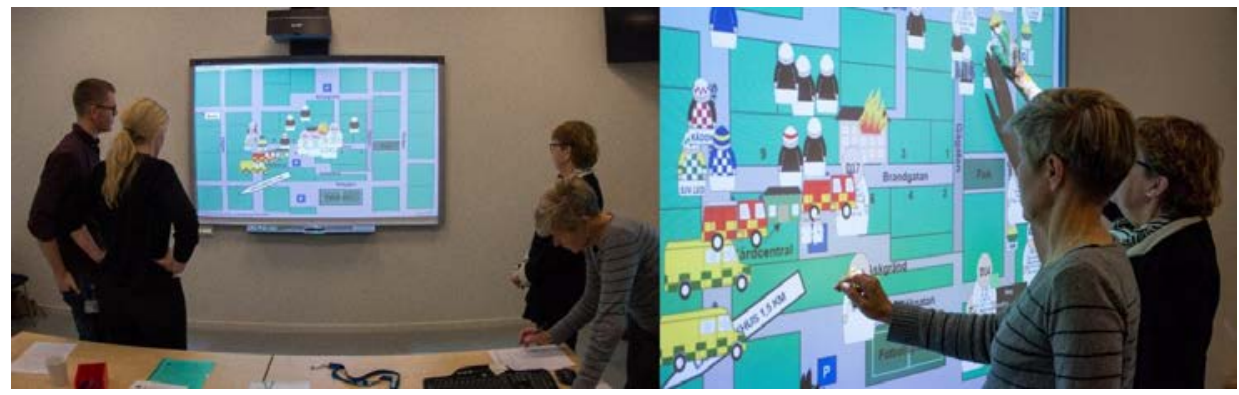

Figure 10: Evaluation of DIGEMERGO. On the left, one instructor (leftmost person) and three participants assess the initial state of the accident site. On the right, two participants interact with the DIGEMERGO touch interface.

\subsubsection{Analysis}

The formative usefulness and usability study, reported in Paper III, indicates that the DigEMERGO prototype is a useful, immersive, and promising simulator. The results from the TAM questionnaires showed that the perceived usefulness of DigEMERGo was rated between "somewhat useful" and "quite useful", and the perceived ease of use scores correspond to "somewhat easy to use". In the free-text answers 
and in post-test interviews, participants often highlighted increased control, reduced instructor workload, and possible future developments - and the educational practices they enable - as the most beneficial DigEmergo features in comparison to ETS. Interpreted as a face-validation study, it demonstrates a level of validity for the DigEMERGo simulator, as most participants perceived the digital system to function similarly to the analog system (i.e., participants claimed that they could complete the same tasks) and the participants were observed to engage in a similar level of immersion to what was observed with ETS (reported in Paper II).

The second evaluation study, the construct validation study, found mixed evidence of simulator validity in emergency management performance between experts and novices. These results and the statistical analyses were reported in full in Paper IV and in Paper VI. In short, the performance measures that pertain to time to action and number of patients processed favored novices and measures of accuracy of correct decisions favored the experts. Novices treated significantly more in-hospital patients, were significantly faster at time to disposal of trauma patients, and cleared a larger number of trauma patients from the ED. However, experts significantly outperformed novices for triage accuracy and disposal accuracy (appropriate destination chosen) of in-hospital patients. Experts also requested additional resources to a greater extent and tended to preserve a higher number of available resources compared to novices. Novices also reported a higher overall experienced workload compared to the experts, especially in terms of experienced demands (both temporal and mental). These results can be interpreted as a successful application of expert knowledge, which would indicate internal and external validity of DigEmergo. Further implications of these mixed results are discussed in section 7.4.2

The third study, reported in Paper $\mathrm{V}$, indicated that using DigEMERGo significantly increased head nurses' general self-efficacy and management skills. In this case, management skill was operationalized as time to treatment for both trauma and in-hospital patients, which was compared between the first and third simulation scenarios. These findings imply that small-scale simulation exercises using DigEmergo could be a potential way to enhance the confidence that the nurses have concerning their task performance, their perseverance when obstacles are met, and their resilience in facing adverse situations. Together, the results of all three studies indicate DigEMERGO's usefulness and validity, which further indicates that distributed cognition theory and the DiCoT methodology can be used for (re)design of human-centered simulations. 
Chapter 5 


\section{CHAPTER 6 \\ RE-INTERPRETING SIMULATION CONCEPTS}

The previous chapter illustrated that distributed cognition theory can be used for studying how a simulator shapes the cognitive system that is being simulated and its abilities to solve cognitive tasks, which can in turn be used when evaluating and designing human-centered simulations. This chapter will explore the distributed cognition perspective as a framework for understanding human-centered simulations from a theoretical perspective. Specifically, core constructs of simulation theory are interpreted in terms of distributed cognition.

\subsection{Human-Centered Simulations as Cognitive Systems}

As a framework for human-centered simulations, the application of distributed cognition dictates that simulation should capture those phenomena of the simuland that form the distributed cognitive system. A simulation should enable cognitive processes in a manner that is similar enough to the way they occur in the simuland, and the distributed cognition perspective views cognition as a computational process that is distributed over physical, social, cultural, and environmental factors. In this view, humancentered simulations are re-creations of cognitive systems. To consider the consequences of this shift in focus, Winsberg's (2015) definition of a simulation from section 2.1.2.3 can be rephrased from a distributed cognition perspective:

\section{Any distributed cognitive system that is believed, or hoped, to bave dynamical behavior that is similar enough to some other distributed cognitive system such that the former can be studied to learn about the latter.}

In terms of the core concepts of simulation, the simuland becomes the targeted cognitive system and its environment. Based on this re-interpretation, the referent is the modeler's knowledge about this system and anything that may affect its functionality. The conceptual model becomes a simplified, selective, and abstracted description of the target cognitive system and its environment. The simulator is perhaps the trickiest aspect to define in terms of distributed cognition. In the everyday use of the word, the simulator refers to the device(s) that enable a simulation session - for instance, a driving simulator often consists of computers, software, monitors, a steering wheel, pedals, and other driving controls. However, a simulator is, by definition, the system that performs the simulation (see section 2.1.2.3), and in human-centered simulations this system includes humans (or other cognitive agents); therefore, these should be included in the term simulator. Consequently, the simulator becomes the composition of the artifacts (i.e., physical, symbolic, mental, etc.) and individuals that comprise the cognitive system - plus its environment - as described by the conceptual model. The definition of a 
simulator suggested by Hancock and colleagues (2010) can thus be rephrased as: a cognitive system that performs simulation. The simulation activity occurs when the simulator cognitive system executes its tasks, thereby generating its processes and behavior. This change of perspective necessitates a reconsideration of what makes human-centered simulations valid and how they should be described.

In the following sections, I will discuss human-centered simulations in terms of distributed cognition using emergency medical triage as an example. As seen in Paper II, the cognitive system uses a number of cognitive tools during triage and management such as physical artifacts in the form of mobile ITdevices, pen and paper, radio, and attachable triage markers (digital or analog). It also uses symbolic or mental artifacts such as rules, communication paths, roles, and triage heuristics and mnemonics. The use of cognitive artifacts and the structure of the cognitive system enable a set of cognitive functions for the systems, such as scaffoldings, information pathways, information transformations, information buffers, communication bandwidths, cues and triggers, and so forth.

\subsection{Interpreting Descriptive Attributes}

Fidelity, scale, and resolution are the three primary descriptive attributes for simulations that describe the selective, simplified, and abstracted nature of simulations. In this section, I will address these attributes of human-centered simulations from a distributed cognition perspective.

\subsubsection{Cognitive Fidelity}

Fidelity, when viewed in terms of distributed cognition, concerns the functional similarity between the cognitive system that is being simulated and the cognitive system in the simuland, rather than physical similarity or experienced psychological similarity. As described in section 2.1.3.1, psychological fidelity refers to the underlying psychological processes that are key to high task performance, but the concept is not itself a theory of what those processes are. A theory of cognition is needed to explain what psychological fidelity is. Distributed cognition is one such theory, which claims that the essential unit of analysis is the distribution of cognition over an artifact-human system, and that this system realizes underlying computational processes through the creation, transformation, and propagation of representational states. From this perspective, elements of physical and psychological fidelity are intertwined and difficult to separate. Rather than assigning a new meaning to the concepts of physical and psychological fidelity, I suggest using the term cognitive fidelity ${ }^{33}$ to refer to the level of fidelity of the cognitive system in simulation. In essence, this term reflects how similar the cognitive system in the simulation is to the corresponding cognitive system in the simuland. This degree of similarity spans over all models and principles of distributed cognition found in DiCoT, such as similarity in paths of information propagation, how and where information is transformed, how agents scan their horizon of observation to maintain situation awareness, how equipment is arranged, how artifacts are used to mediate cognition, how the system tracks its goal state, etc. Theoretically, having perfect cognitive fidelity would imply that the structure and the functions of the cognitive system acting in the simuland are accounted for and are perfectly replicated in the simulation. A simulation that enabled none of the functional characteristics of the target cognitive system would have zero cognitive fidelity.

\footnotetext{
${ }^{33}$ This term is also used by Hochmitz and Yuviler-Gavish (2011) However, they use the term like psychological fidelity
} 
Even though physical artifacts are key to cognitive fidelity, high cognitive fidelity does not necessarily require high physical fidelity. As cognitive fidelity is a theoretical measure of functional, not physical, similarity, determining the required level of physical fidelity is not a straightforward task. Therefore, capturing physical similarity has no inherent value; rather, it is capturing the functionality mediated by those physical artifacts and tools that is of interest — and different tools can function similarly despite different physical specifics or levels of technological sophistication. For example, real-world medical triage uses color-coded physical tags (often bracelets or neckbands) that are attached to the patient. These tags serve multiple functions, such as representing, communicating, and buffering the patient's triage status, thus signaling the patient's medical state. For a simulation, these triage-functions are important to capture. In ETS simulations, small adhesive labels are used as triage markers. In Paper II, we found that even though these labels do not have the same size, shape, or attachment mechanisms as real markers, they mediate similar essential distributed cognitive functions. In this example, color is the load-bearing property on which the triage-functions rest - not the marker shape. Therefore, changing shape or size does not affect the marker's core functionality. However, changes to such properties can still affect the cognitive system and its abilities. For instance, changing the attachment mechanism of the markers alters the bodily motor-procedures that are involved in assigning triage, which communicate subtle body signals within the distributed cognitive system.

As opposed to physical fidelity, the level of cognitive fidelity is hard to determine at a glance. Study and analysis of the simulation is required - such as the DiCo'T-analysis presented in Paper II. It is important to remember that fidelity, including cognitive fidelity, is not related to the intended purpose, goals, or functionality of the specific simulation - it is only an estimation of the degree of similarity to reality. Therefore, (excessively) high cognitive fidelity can hinder the purpose of the simulation in a similar manner to the way that (excessively) high physical fidelity can. This line of argument follows Alessi (1988) and others who claim that high levels of physical fidelity can be beneficial for experts, but less useful or even an obstacle for novices. It seems likely that high cognitive fidelity simulations can be beneficial for training or assessing expert skill, but could form a task environment that is too complex, which might overwhelm novice learners.

In the literature, it is argued that the participants' perception and recognition of objects in the simulated task is important for psychological fidelity. This subjective experience can be linked to other concepts of simulation, such as participant engagement, motivation, immersion, simulator believability, and suspension of disbelief (see for instance Drews \& Bakdash, 2013; Gray, 2002; Witmer \& Singer, 1998). Using cognitive fidelity, it can be argued that these aspects of subjective experience may be a result of establishing a cognitive system in simulation that is similar enough (and thus subjectively familiar to cognitive agents) to the cognitive system of the simuland. It seems reasonable that working in an environment that does not function as one is used to would break the suspension of disbelief, regardless of how physically real it appears. In Paper II, we found that ETS could create high levels of engagement despite its low physical fidelity - the simulation looks nothing at all like reality. This thesis argues that this is because ETS recreates the cognitive system, its functions, and its environment in a manner that is cognitively similar enough to the simuland. 


\subsubsection{Resolution and Scale}

Resolution resembles fidelity, but concerns the level of detail in the simulation rather than its similarity to reality. From a distributed cognition perspective, resolution reflects simplifications made to the cognitive system, its constituent elements, its environment, and the information flow in the system. For example, analysis of the cognitive system in the simuland can reveal information flows over multiple paths, for instance through a series of different artifacts, before it is re-transformed or acted upon. A simulation with high resolution would enable all of these information paths, whereas one that has been simplified to include only one or two paths would be considered lower in resolution. Resolution that is too low could lead to validity issues, given the purpose of the simulation, because the cognitive system would not function as intended. Some simulations may only need to capture the "bare bones" cognitive functions, whereas others might require a more detailed recreation of the cognitive system. As with fidelity, simulations for introductory training in emergency medical triage might want to focus on the core triage task and reduce or simplify other cognitive tasks that might otherwise overwhelm novice participants.

Scale is an attribute that relates to the selection of functions and elements from the simuland that are to be included in the simulation. From a distributed cognition perspective, scale refers to where the functional boundaries of the cognitive system are drawn, which separates the system from its environment. Scale is thus a description of the size of the cognitive system that is simulated. The scale that is required for a simulation to fulfill its purpose is relative. For the triage-training example, the boundaries should at least be drawn to include the patient (state), the triage system, the tools for triage, and the medical personnel. Regional management, other agencies, additional emergency medical roles, ambulances, transports, treatments, etc. can be simulated as part of the environment that provides the triage cognitive system with inputs and outputs. However, in a simulated assessment of emergency medical capacity during a disaster the scale might involve other organizations and agencies. It would probably be necessary to simulate these fully, as opposed to representing them as simply a source of environmental inputs. A large-scale simulation thus implies a large cognitive system that includes multiple elements, or that alternatively involves a number of cognitive systems that interact with each other to solve a cognitive task. A larger scale than the purpose of simulation required would not make the simulation more valid or effective. Therefore, a large scale is not a goal in itself. However, a scale that is too small for the purpose of the simulation could mean that the simulation would not capture the intended cognitive system and its functionality, which would imply validity issues.

\subsection{Interpreting Validity}

Validity reflects the degree to which the correct things are captured in a correct manner, and also measures the accuracy of the results that are generated by the simulation. Adopting the distributed cognition perspective implies that the most important aspect of a human-centered simulation is to identify and capture the correct cognitive system, including its distribution, properties, and environment. Through abstraction, simplification, and selection, the goal is that an appropriate version of this target system should be re-created in a manner that will serve the intended purpose of the simulation. It might be tempting to conflate validity of human-centered simulations with high cognitive fidelity. The concepts do partly overlap, as a valid human-centered simulation will require a certain degree of cognitive fidelity. However, the key difference between cognitive fidelity and cognitive 
validity lies in the intended purpose of the simulation: validity is purpose-dependent and fidelity is not. Achieving the highest possible fidelity would imply not performing any abstractions, simplifications, or selections when modeling the cognitive system of the simuland, whereas validity is about performing these tasks in a correct and accurate manner. ${ }^{34}$

As seen in section 2.1.3.2, there are many sides to validity. In the following section, the distributed cognition perspective will be deployed to reinterpret the validity framework developed by Feinstein and Cannon (2001, 2002). However, first some slight adjustments to the framework will be presented. First, Feinstein and Cannon discuss the achieved transfer of training as educational validity, which in the case of training simulators reflects the intended result and desired output of a simulation, section 7.2 will discuss this further. If we are to consider other human-centered simulations (or simulations in general), I suggest using the more generalizable term application validity to reflect the degree to which the simulation achieves its intended results - whether they be positive transfer of training, accurate predictions, or something else. Furthermore, I want to address overlaps between the validity concepts, especially between representational validity and internal validity, and between applicational validity and external validity. The concepts are intended to be considered on different dimensions: the development phase of the simulator and the simulator structure. However, considering internal and external validity as properties of the underlying conceptual model, and representational and applicational validity as belonging to the simulator and the simulation results, makes these four aspects of validity more distinct and easier to apply. Naturally, the aspects of the conceptual model can still be validated through an ordinary validation process in the simulator. Some aspects of the conceptual model are first accessible when the simulator is executed, for instance the external validity property of whether a participant can recognize the simulated situation and apply her expertise in this environment.

\subsubsection{External Validity}

High external validity requires accurate selection of key components of the simuland and determining whether they have been successfully captured. Determining external validity involves verifying that the correct cognitive system has been selected and identifying where its boundaries are drawn. As previously discussed, these boundaries are based on which functions, rather than which elements or what physical structure, to include. For instance, in Paper II the grouping and special allocation of ETS patient figures were identified and deemed to be essential properties of the cognitive system's triage functionality and its modus operandi, and these properties were thus selected to be retained in redesign. System demarcation is relative and open to subjective judgment regarding which elements in the system are included in the cognitive system, and which are parts of the system's environment that provide it with inputs and react to its outputs. The environment can consist of, for instance, teachers, confederates, or automated parts of the simulator device (e.g., rules or artificial intelligence agents). Another aspect of external validity is that real-life behavior and individuals' expertise should be transferrable into the simulation. Ideally, being an expert in reality would make you an expert in simulation as well - and vice versa. For this, distributed cognitive structures that support expertise should be adequately similar in the simulated setting to the way they are actually arranged in the simuland setting.

\footnotetext{
${ }^{34}$ Not performing these tasks is in and of itself a contradiction, as modeling is abstraction, simplification, and selection Perfect fidelity would be a product of replication or copying, rather than modeling and simulation
} 


\subsubsection{Internal Validity}

Internal validity is about whether the correct aspects of the simuland are simulated and if they are simulated in a correct manner. From the distributed cognition perspective, this is a question of whether the cognitive system functions as intended or not. There is a conceptual overlap with external validity with regards to whether the correct aspects are simulated, that is, whether the correct selections have been made. Therefore, the focus should be on the way in which elements in the simulation are abstracted and simplified. Internal validity thus refers to whether the selected functions of the simuland cognitive system are simplified and abstracted in such a way that similar enough functionality is enabled and accurate and coherent output is produced (e.g., in terms of performance). Coherence, which relates to the reliability of simulations, means that the cognitive system being simulated operates in the same manner over multiple simulations; for instance, that information moves and is transformed along the same paths.

Retaining functions in simulation, such as the identified grouping and spatial allocation function of ETS in simulations, does not imply that identical representations must be used, rather it is only necessary to use representations that enable the same functionality. With regard to representations there is a conceptual overlap with representational validity, which considers the correctness of simulated element representations. To clarify, internal validity should reflect whether the correct representations (manner of simulation) have been chosen, and representation validity should consider whether these representations have been correctly implemented. For instance, the choice to use digital whiteboards to represent settings, patients, and resources in DigEMERGO should be considered an aspect of internal validity; the identified need for them and whether they actually capture the targeted functionality is external validity; and the design and layout of these digital whiteboards, patients, and resource figures (including their interaction) should belong to representational validity.

\subsubsection{Representational Validity}

As in the original definition by Feinstein and Cannon (2002), representational validity is, from a distributed cognition perspective, the correctness of representations used in simulations. The representations that are used for elements and information in a simulator can mediate properties of the cognitive system, and representational transformations are parts of the problem-solving process. For instance, cognitive cues and scaffoldings often rely on representational properties. Again, using the color codes in triage as an example, a valid representation of triage tags captures those functions of the cognitive system that enable choice, assignment, and interpretation of triage levels. For such functions, principles from the physical layout model and information flow model are critical, such as space and cognition, naturalness principle, situational awareness, horizon of observation, information buffering, and behavioral trigger factors. Additionally, to capture the functions of assigning triage, the medical state of patients must be assessable. This means that it is important that the representation of the patient's medical state and need for medical intervention can be accessed by the cognitive system so that an accurate decision-making process can be initiated. As the medical state only needs to be accessed, and not medically assessed in a manner similar to reality, the patient's physical features can be abstracted. On the other hand, if one is interested in capturing the functions of the cognitive system associated with performing the medical assessment itself, a higher level of physical fidelity of the patient would be required for representational validity. 


\subsubsection{Application Validity}

The degree to which a simulation solves the problem for which it has been created reflects its applicational validity. This concept of validity is about the results of applying human-centered simulations, and not (directly) about the construction of a simulator or the processes that occur during simulation. Regarding transfer of training (educational validity), this can, in some cases, be interpreted as internalization of practices that would otherwise be distributed in the cognitive system. However, in general application validity does not explicitly concern the cognitive system that is being simulated in the way that the other concepts of validity do. I will discuss what is required for a human-centered simulation to exhibit application validity in section 7.2. 
Chapter 6

68 


\section{CHAPTER 7 \\ DISCUSSION AND CONCLUSION}

This chapter will discuss the research questions, research approach, and results of the thesis. In doing so, the chapter will frame the results as products of either technological research or science, which in turn implies who is mostly likely to use the results and how they should be interpreted. Furthermore, limitations of the thesis and its constituent papers will also be discussed, and thoughts for the future will be presented. The chapter ends the thesis with a section of conclusions.

\subsection{Addressing Research Questions}

One overarching claim of this thesis is that capturing the appropriate cognitive processes of the simuland is the most important aspect of human-centered simulations. This requires a theory or framework of cognition. Much simulation research is influenced by the classical view on cognition, according to which individuals can be easily transferred between contexts with little or no effect on cognition. The cognitive environment and the distributed nature of cognitive activities are thus often disregarded (or not theoretically well-understood) in the simulation literature. From a distributed cognition perspective, not accounting for such distributed cognitive aspects in a simulation can cause validity issues for human-centered simulations, as the targeted cognitive activities of the simuland may not be simulated accurately. In the following sections, the set of research questions presented in section 1.1 will be addressed explicitly.

\section{R.Q.1. How can distributed cognition be used for evaluation and (re)design of buman-centered simulations?}

This thesis started with the hypothesis that distributed cognition can be used as an analytical framework for studying and evaluating human-centered simulations. In Paper II, simulated environments are studied from a distributed cognition perspective, illustrating that the cognitive activities and processes of the distributed cognitive system in the simulated environment can be identified and analyzed. The paper further illustrates how a simulator's design can affect the functions and properties of this cognitive system, which in turn affects not only the performance of the system, but also whether the system solves cognitive tasks in a manner that resembles problem-solving in reality. Therefore, we conclude that distributed cognition can be used to evaluate the validity of a simulation. Furthermore, knowledge of the cognitive processes and mechanisms that are essential in reality can guide the design of valid and effective human-centered simulations. Paper II and Paper III exemplify this through a digital redesign of an existing analog simulator based on a distributed cognition-based analysis. Even though Paper II studies another simulator instead of the real simuland, the principle is the same since 
cognitive processes and mechanisms that are considered to be of importance for the analog simulator are transferred into the design of the new digital simulator.

\section{R.Q.2. Is the DiCoT methodology a useful approach for studying and designing simulator environments?}

In order for DiCoT to be considered useful for the study and design of human-centered simulators, it should be able to identify and analyze properties of the cognitive systems that are being simulated. It should also be able to guide the design of simulators in ways that retain or capture the relevant cognitive systems of the simuland. In Paper II, the DiCoT methodology was used to provide an analysis that identified essential properties of distributed cognitive systems in ETS simulations. This allows for comparisons between a DiCoT analysis of the simulation and a DiCoT analysis of the simuland, which in turn can reveal aspects of validity of the cognitive system that is being simulated. Furthermore, as demonstrated in Paper II and Paper III, de-constructing cognitive systems using DiCo'T can also identify validity issues in simulations without any direct comparison to an analysis of the simuland. Based on referent knowledge of the simuland, the DiCoT analysis of the simulation can identify cognitive processes that are side-effects (i.e., byproducts) of the simulator design and setup, which thus do not occur in the simuland.

Regarding the design of human-centered simulations, Paper II and Paper III illustrates how a DiCoT analysis of human-centered simulators can be used to motivate the re-design of simulators through identification of validity issues and areas for potential improvement. Furthermore, a DiCoT analysis can also guide the (re)design of human-centered simulations by highlighting the aspects of the target system that are important to retain, and which aspects can be changed or improved. DiCoT provides no explicit aid for simulation modeling in terms of abstractions, simplifications, and selections. However, DiCoT facilitates an understanding of which elements of the simuland (physical, social, representational, cultural) are key to the functionality and performance of the cognitive system, and subsequently which elements should be selected, abstracted, and simplified so as not to lose this functionality. Based on the above results, DiCoT can be considered a useful methodology for both the study and the design of human-centered simulations. Furthermore, DiCoT can be regarded as a potential qualitative validation approach to human-centered simulations.

\section{R.Q.3. How does distributed cognition further our understanding of human-centered simulation?}

This thesis claims that human-centered simulations are a subset of simulations where humans are engaged in cognitive activities that are hoped to be similar enough to reality to serve a specific purpose. Adopting the distributed cognition perspective on cognition provides a different definition of what cognition is and how humans engage in cognitive activities in the real world (in the wild). This, in turn, enables a re-interpretation of what human-centered simulations are: re-creations of distributed cognitive systems. The distributed cognition perspective does not only provide a means of studying human-centered simulations, it also facilitates a different interpretation of what human-centered simulations are, how they should be analyzed, and what makes them successful. Here, it is relevant to mention a common notion in the distributed cognition literature: that cognition in the wild is different from cognition in captivity (i.e., in laboratory settings). This is because the cognitive environment is different in everyday real-life versus in laboratory settings; thus different cognitive systems emerge in the wild versus in captivity. Following this line of thought, one could ask if simulations should be 
considered to be cases of cognition in captivity, and whether this is a problem when, for instance, providing training on emergency management in a simulated environment? In principle, a simulation is a form of cognition in captivity that will differ from cognition in the wild, because any simulation is, by definition, a simplification and abstraction from reality. However, this thesis argues that selections, simplifications, and abstractions do not have to be a problem if the simulated environment is created with the notions of cognitive environments and systems in mind. Through the distributed cognition perspective, the simulation environment can preserve the core cognitive structures that operate in the wild, making the cognitive processes that are being simulated similar enough for its purpose and thus valid.

\section{R.Q.4. How can central theoretical simulation constructs be interpreted and explained through the distributed cognition perspective?}

When adopting a new interpretation of what human-centered simulations are, the characterization of descriptive attributes and validity of human-centered simulations also change by extension. Section 6.2.1 describes how fidelity - which is the prime measure of similarity between a simulation and the simuland - becomes a theoretical measure of the degree to which the cognitive system in the simulation resembles the cognitive system in the simuland. Similarly, resolution is defined as the level of detail of the cognitive system that is being simulated. This means the degree to which the cognitive system being simulated includes elements (within the boundaries) from the cognitive system of the simuland. It does not, however, say anything about how they are implemented in simulation. Scale refers to where the boundaries of the distributed cognitive system are drawn. This is important for determining the cognitive system that is to be recreated in simulation and what is to be regarded as the system's environment.

In section 6.3, Feinstein and Cannon's (2002) framework of validity was interpreted using distributed cognition to characterize what is important for valid human-centered simulations. Validity refers to the degree of correctness or accuracy with which the cognitive system is implemented in simulation, which stands in relation to the purpose of the simulation. External validity refers to the degree to which the most important elements and functions of the distributed cognitive system are selected for simulation. Internal validity can be seen as the degree to which correct simplifications and abstractions of the cognitive system are made and how they are composed, so that the distributed cognitive system in a simulation functions and interacts in a consistent manner that is similar enough to reality. Representational validity is related to internal validity, as it concerns the correctness of representations used in a simulation. A correct representation succeeds in enabling or mediating intended functionality of the distributed cognitive system. Application validity, which is a broader interpretation of educational validity, is the degree to which the simulation and its results are valid for its intended purpose (application). From the distributed cognition point of view, application validity applies to the correctness of the outputs from, or changes to (e.g., learning), the cognitive system.

\section{R.Q.5. What simulation components contribute to effective emergency medical management training according to the distributed cognition perspective?}

This thesis builds on the growing literature that claims that physical fidelity is not the most important aspect for successful SBT. Rather, important for effective and valid training is enabling and mediating cognitive processes similarly enough, for instance, how information flows and is transformed over 
artifacts and team members. Distributed cognition is, in this way, especially suitable for identifying factors that relate to effective training of non-procedural skills. To give an example, to train off-site medical management it is important to capture the processes and functions the cognitive system use in making sense of reports from the field. In Paper I, it was observed that reports of a possible major medical emergency required information to propagate through the cognitive management system, triggering several re-transformations, which in turn increased workload. By capturing such processes, similar work procedures and skills are reinforced and honed through simulation practice. The DiCoT analysis in Paper II illustrates that ETS enables training of such cognitive skill using low physical fidelity. However, this is not to say that physical fidelity is irrelevant from a distributed cognition perspective. It depends on the type of processes a simulation is meant to capture and what the intended purpose of the simulation is. For instance, experts can interpret fine-grained visual patterns of information when, for example, visually scanning an accident site. Thus, advanced and sophisticated representations of reality would be required for capturing such professional vision. Moreover, it is important to understand which cognitive processes the simulation should re-create and their role in the simuland, as this will affect the required levels of fidelity, scale, and resolution.

\subsection{Application of Human-Centered Simulations}

It is relevant to take a step back and consider what makes human-centered simulations useful as a technique and how aspects of validity relate to usefulness. In Paper II, we claimed that a useful training simulation is one that exhibits educational validity (i.e., a simulation that generates a positive transfer of skill and knowledge). However, this statement requires further elaboration. For a simulation to be a useful technique it must be functional - which means to produce its intended results - and some user must also exist that finds the simulation helpful for solving a practical problem.

In section 6.3.4, I have argued that education validity is a special form of a more general application validity, which addresses the degree to which a simulation (or simulator) produces its intended results and thereby fulfills its purpose. This seems similar to functionality of a technique, see section 2.1.2.1. Adding to the confusion, Feinstein and Cannon (2002) argue that internal validity address the extent to which simulation functions as intended. This brings in to question whether a functioning simulation is one that exhibits applicational validity or internal validity - or both. To address this question, it must first be clear what the results of a simulation refers to. Results are, in this sense, not necessarily the output data from a simulation. Rather, they can be seen as the outcomes of applying a simulation technique (including its output) to some real-world problem. "Producing the intended simulation results" can be interpreted in two ways. First, that a simulator should produce processes and outputs that are similar enough to the simuland: being an accurate simulation, which translates to exhibiting internal and external validity. The second is that, through use of the simulator, the intended purpose of the simulation is achieved (i.e., application validity).

To illustrate the differences between the two interpretations, consider a driving simulator used by two different individuals with different goals. One individual wants to predict rush-hour traffic accidents and the other wants to become a better rush-hour driver. The simulator produces valid traffic behavior that reflects what would be observed in reality, but it suffers from a flawed user interface that is difficult to operate when driving. For both users, the simulation fulfills the first interpretation, as it produces a simulation of traffic and all the functions of a car in a way that is similar enough to reality - the 
simulation is internally and externally valid in those respects. For the first user, the simulation fulfills the second interpretation as well, because the user can successfully use the simulation to predict accidents - thereby achieving the intended purpose. Thus it exhibits application validity. For the second user, however, the simulation would not fulfill its purpose, as the flawed driving experience would cause the user too much stress, thus hindering the user from becoming a better rush-hour driver.

The example above illustrates that simulating the simuland correctly and fulfilling the intended purpose of simulation do not necessarily have to be the same thing. Therefore, it is not sufficient to simulate the correct things for a simulation to exhibit application validity. For human-centered simulations, this means that re-creating a similar enough cognitive system is insufficient for application validity. It also means that a simulation may exhibit application validity for one intended purpose, but not for another. Returning to functionality: it seems reasonable to claim that the simulator functions in producing a simulation of rush-hour traffic, but not that it functions as a training simulator for driving. In answer to the question stated earlier, both technological functionality and applicational validity reflect whether a simulation fulfills its intended purpose, and therefore it seems fair to conclude that these two concepts are equal. Internal validity, on the other hand, refers functioning in a "correct simulation" -sense, which as we have seen is not the same thing. The difference lies within the intended purpose for which the simulation is used.

What about usefulness? In the example above, usability of the human-centered simulation was a factor that affected its usefulness. For all simulation interfaces, usability is important, but the relationship between usability, functionality, and usefulness requires further elaboration. There are, for instance, no aspects of usability (that affects validity) to consider during execution of a constructive simulation with no human interaction. If such a simulator is correctly modeled and implemented, and the underpinning theories are true, the simulator will produce its intended results (frequently enough) and thereby function appropriately - even if the results are outputted in a way that makes them unusable. Unusable output would, however, be devastating for the usefulness of such a simulation. It seems that usability and functionality are separate concepts for different simulation techniques. I argue that functionality and usability of simulations relate to each other based on the roles that users have, which differ for constructive simulations and human-centered-simulations. As Figure 11 illustrates, users are parts of human-centered simulations if they are parts of the cognitive system that constitutes the simulation. However, users may also be those who use any outputs from the simulation or observers of the simulation (dashed figure). Furthermore, different user roles within a human-centered simulation relate differently to usability. In training simulations, for example, some users are participants in the simulation who are intended to learn (green figure), while others might be teachers or confederates who are there to instruct (red figure). 


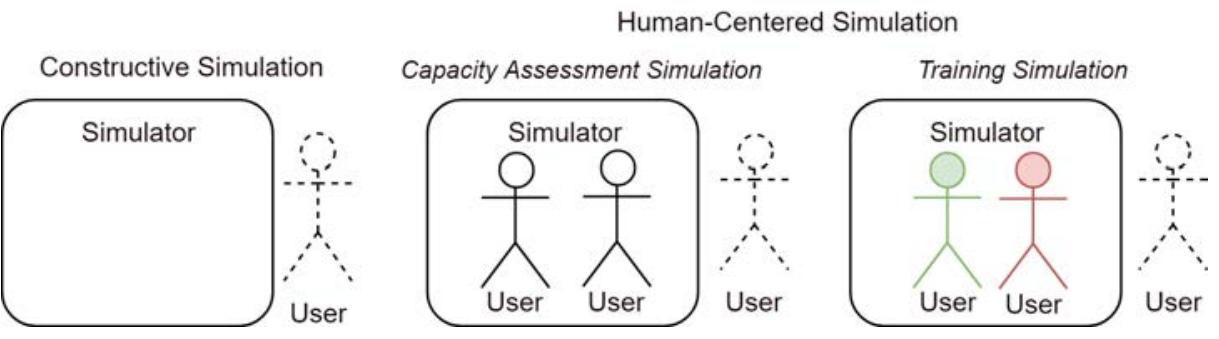

Figure 11: Examples of different user-types in buman-centered simulations.

If the interfaces and artifacts that these users engage with are of too (unintended) poor usability, the cognitive system will falter and the simulation should not be considered functional. In other words, it is not usability per se that is important for functionality, but rather establishing a working cognitive system, which may require a certain degree of usability.

Beyond functionality, other aspects of a human-centered simulation contribute to whether it should be considered useful. For instance, if a simulation produces uninteresting or inapplicable (yet correct) results, or is too impractical to execute (e.g., due to high costs), or if no user wants to - or can - use it (e.g., due to trust, motivation, or usability issues as demonstrated earlier) the simulation would not be useful. The distributed cognition perspective offers an explanation to why and how human-centered simulations function. Obviously, functioning and useful human-centered simulations have been constructed previously, without using distributed cognition. However, there is a difference between building useful techniques and understanding why those techniques are functional and useful. Perhaps an incomplete understanding of the workings of human-centered simulations and why they produce the results they do has been a contributing factor to the overemphasis on physical fidelity (naïve realism).

\subsection{Contributions as Products of Technological Research and Science}

The results of this thesis apply both to the technological and the scientific areas of the simulation discipline. Chapter 5 mainly provides contributions to technological research, for several reasons. First, the chapter discusses uses, evaluations, and design of simulations, not theoretical understanding of the nature of simulations. Second, the distributed cognition perspective is explored as a tool to study and evaluate human-centered simulations. In this way, the DiCoT analysis of the ETS simulator, as well as the design of the DigEMERGo prototype, serve as two cases that investigate the usefulness of distributed cognition as a tool to study and design human-centered simulations. Those results are important for technological simulation research as well as expanding the use of distributed cognition. Furthermore, the DigEmergo simulator and its design are per se a product of technological research, as the ultimate goal of DigEmergo is to be useful for solving real-world problems outside of research (i.e., for training in and assessment of emergency medical management). In Chapter 6, however, a re-interpretation of human-centered simulations as distributed cognition activities is presented. The goal of Chapter 6 is to generate knowledge on how human-centered simulations can be seen, their inner workings, and how their theoretical constructs can be interpreted. The suggested definition of human-centered simulations 
in Chapter 6 is, in a sense, a theory of what human-centered simulations are - distributed cognitive systems. This chapter does not, however, consider the usefulness of such an interpretation.

The usefulness of distributed cognition as a tool for studying human-centered simulations can be tested, either subjectively or by showing that the simulation-related products created using this tool achieve some desired goal, such as effectiveness, validity, or usefulness. It is more difficult to prove or disprove the distributed cognition interpretation of what human-centered simulations are. However, this interpretation can be rephrased to illustrate the appropriateness of such an interpretation. This can be done by claiming that human-centered simulations reflect cognitive processes in general - not distributed cognitive systems in particular. When rephrased this way, the theory of what humancentered simulations are now deals with similar enough cognitive properties and processes in general, which is not bound to distributed cognition theory specifically. Therefore, if distributed cognition were to be strengthened or discredited as an explanatory theory of cognition, which is debated ${ }^{35}$, it would strengthen or discredit distributed cognition as a theory of (or framework for) human-centered simulations as well.

A caveat to address here is that the human-centered simulations studied in this thesis are obvious cognitive situations. However, there are simulations in which humans are central that focus mostly on motor activities, for instance, surgery simulation (Dawe et al., 2014). The question then becomes whether or not such simulations should be treated as something apart from human-centered simulations. I think not. As Hollnagel and Woods argue (2005, p. 24): all work is cognitive. This means that everything we do requires cognition to some degree, and therefore there is no need to distinguish between cognitive work and non-cognitive work. Naturally, different activities are more or less cognitively demanding or complex. A less complex simulated activity would therefore require a less detailed cognitive analysis for evaluation, which can be achieved by using a subset of the DiCoT principles that are relevant to the simulated task.

\subsection{Limitations}

Throughout my doctoral studies and writing this thesis, my perspective has changed somewhat due to a deeper understanding of both the simulation field and distributed cognition. The main premises or ideas have not changed, but rather how they are phrased. For instance, Paper III discusses the concept of "cognitive validity" as an explicit measure of the correctness with which the cognitive system is recreated in a simulation. Although I still see the value of using such a term to refer to the accuracy of cognitive processes in simulation, the interpretation of human-centered simulations as cognitive systems makes this term rather underspecified - as all forms of validity of human-centered simulations reflect cognitive properties in some way (with the possible exception of applicational validity). Therefore, the structure provided by Feinstein and Cannon was adopted and modified to emphasize aspects of the cognitive system.

\subsubsection{The DigEmergo Design Case}

The DigEmergo design case used in this thesis is limited. I argue that distributed cognition can be used to guide the design of simulators by analyzing the distributed cognitive system of the simuland.

\footnotetext{
35 See for instance Adams \& Aizawa (2010), Elpidorou (2014), and Adams and Garrison (2013, 2014)
} 
However, the DigEmergo simulator was designed based on analysis of simulations with the ETS simulator, which cannot be considered to be the real simuland. The severity of this limitation is debatable. On the one hand, distributed cognition was used to analyze the cognitive system present in one setting (ETS simulation), which enabled identification of core mechanics and shortcomings of this system. Furthermore, distributed cognition has previously been shown to be valuable for studying cognitive systems in real medical settings (i.e., the real simuland). Thus, the conclusion that distributed cognition can be used to analyze the simuland setting and inform the design of a simulation seems unproblematic. On the other hand, as another simulation (ETS) was the basis for the DigEMERGO simulator design, many abstractions, simplifications, and selections had already been made with regard to the simuland. Thus, the modeling stage of DigEMERGO was in part performed during the modeling of the ETS simulator. In this case, the DigEMERGo modeling became an exercise in evaluating and improving upon previous modeling assumptions, design choices, and implementations that were already in use in ETS. Therefore, the case is limited in the ways it tests distributed cognition's and DiCoT's ability to inform selections, abstractions, and simplifications for simulations.

\subsubsection{Mixed DigEmergo Validation Results}

The comparative expert-novice validation approach reported in Paper IV and Paper VI provided an indirect test of whether the use of distributed cognition in the design of DigEMERGo had successfully captured such properties of ETS in a correct and valid manner. The results showed mixed performance results. Experts outperformed novices on key quality measures, such as triage and disposal accuracy of in-hospital patients, and they anticipated a need for additional resources. However, triage and disposal accuracy for trauma patients did not reveal any differences. The novices were also faster at patient disposal for in-hospital patients and outperformed the experts in terms of number of patients treated and number of patients disposed of. There are several possible explanations for these results: First, that DigEmergo did not sufficiently capture and convey the emergency department setting and its supporting tools to the participants, with the result that the established cognitive system in the simulation was not similar enough to reality. Second, the DigEMERGO prototype was not sufficiently usable for the expert users, thus hampering performance. Third, the measured performance variables do not necessarily reflect validity. Fourth, flaws in the study setup (such as insufficient pre-training) introduced confounding variables that muddled the results. It is probable that elements of all of the listed explanations affected the outcome of the validation study.

To a large degree, the performance measures used seem to reflect usability issues and experience with the digital prototype. This effect could have been reduced by extended pre-test training sessions with DigEmergo. It is notable that all participants, especially the experts, scored below what would have been expected in triage accuracy for the trauma patient group. This was probably also caused by flaws in the study setup. Too few trauma patients in need of triage were included and a confounding variable was found post-test in the triage system that was used, which complicated the question of what would be considered accurate triage of trauma patients in a traffic accident. Using time and quantities as an indicator of surge capacity management performance - and thus also for validation - is debatable. Faster performance does not necessarily equal better performance. An incorrect triage assessment is always incorrect no matter how quickly it was performed. In some cases, slower performance can even be desirable; for instance, longer time to treatment for patients with minor injuries can indicate that more severely injured patients were prioritized or anticipated to arrive at the ED. Furthermore, clearing 
the ED as fast as possible, which occupies resources in this scenario, might seem favorable, but keeping resources available in reserve for as long as possible might reflect preparedness for additional incoming trauma patients. Such thinking would reflect a deeper system understanding and ambition to achieve high quality of care. Additionally, for this specific study, the simulation model was further simplified as the setup removed the need for participants to select treatments for each patient. If the required resources (e.g., nurses, doctors, and rooms) were provided by the participant the DigEMERGO simulator automatically selected an appropriate treatment plan. This study design choice was made to lessen focus on medical knowledge and to favor management skills. It is possible that this decision, in combination with other simplifications of the ETS and DigEMERGo systems, provided management tasks that were too simple, reducing the possibility of distinguishing novice and expert users from each other in a meaningful way - especially when using speed and quantity as performance measures.

Flaws in the study design and selected measures in combination are not a reason to discredit the validity of the DigEmergo simulator, but they do call into question the strength of the validation study. Results from Paper III and Paper V indicate usefulness, immersion qualities, and application validity of DigEmergo. Additional validation studies of the DigEmergo simulator could provide significant and conclusive results. If it is the case that there are validity issues with the DigEmergo simulator that prevent it from being able to separate experts from novices, it is important to consider why. The thesis and its constituent papers assume a priori that the ETS simulator is valid. Therefore, either DigEMERGO fails to capture whatever makes ETS valid, which would weaken the claim of distributed cognition as a framework for simulator design, or the assumption is faulty.

\subsubsection{Alternative Research Approaches}

As with any research project, there are many things that could have been done differently and there are many more studies that should be conducted in this area. One gap is that no DiCoT analysis of the DigEMERGo system was conducted within the thesis. The primary reason for this was to avoid circular reasoning, since evaluating the DigEMERGO system with the same framework that was used to construct the system would be biased and circular. Therefore, I wanted to use established methods within the simulation field and human-computer interaction for evaluation and validation of the DigEmergo system. Such methods would also reveal positive outcomes, in terms of simulation results, of adopting a distributed cognition perspective. That said, a DiCoT analysis of DigEMERGo would be an interesting and valuable additional study that could illustrate differences in distributed cognitive processes between analog ETS simulations and digital DigEMERGO simulations. Such a study could more clearly highlight what has been gained and lost in translation from an analog to a digital simulator, and analyze whether deliberate changes made from ETS to DigEMERGo had the intended effects.

An alternative approach to the design of DigEMERGo would have been to consider the system to be unrelated to ETS, and to treat ETS like a true simuland rather than as a candidate for re-design. In this way, less of the ETS structure would have been adopted for the purpose of retaining familiarity with the system. It would have been more appropriate still to study additional real emergency medical management situations, as in Paper I, with DiCoT and build a simulation environment based on that analysis. Furthermore, there are other analytical approaches to distributed cognition that could have been used in this thesis, such as the three-level framework (Garbis, 2002). A possible benefit of such 
an analysis would be the inclusion of cognitive processes at the organizational level, which is not emphasized in DiCoT.

\subsection{Thoughts for the Future}

In this section, I will briefly discuss the implications of this thesis for future research. There are a number of studies of interest to pursue based on this thesis. Specifically, developments of DiCoT for simulation research and extensions of the DigEMERGo system are discussed.

\subsubsection{Additional Validation Studies of DigEmergo}

A revised expert-novice validation study could provide conclusive results regarding internal, external, and representational aspects of validity. An additional approach to consider would be a so-called Turing test validation. Turing test validation is an established validation method in which subject matter experts are tasked with distinguishing simulator output data from empirical simuland data. If they cannot discriminate between simulated and real data, the simulator is said to pass the Turing validation test (Law, 2009; Sokolowski and Banks, 2010). The novice-expert validation approach is a form of Turing test, in which the simulator (or the analysis of simulation results) judges user performance. This tests the simulator's ability to successfully distinguish between experts and novices, which would indicate that the simulator can accurately judge performance. Using a standard Turing test validation approach would be appropriate for validation of parts of the simulation, for instance, validating injury distributions of accident scenarios in simulation. It can also be used to validate the results from an entire simulation; for instance, in the assessment of surge capacity output data (e.g., time to treat patients, number of casualties, triage distribution). In this way, a set of impartial experts could judge whether the data were results from a simulation or a real scenario. Furthermore, additional study of applicational validity would be of interest, using DigEMERGO as both a technique for training as well as a technique for assessment of skill and surge capacity.

\subsubsection{Development of DiCoT}

There are a number of ways in which DiCoT can be developed as a research method for humancentered simulations. As previously mentioned, DiCoT is intended as a general method for studying distributed cognition in work settings, which does not provide explicit support for studying humancentered simulations specifically. DiCoT could be customized for studying human-centered simulation. Such a version of DiCoT could provide different principles depending on the study purpose: whether the analysis was being performed to support the design of a simulator, to provide referent knowledge of the studied cognitive system, or to validate simulations. For validation purposes, for example, sets of principles can be tied to aspects of simulation validity, which would enable more standardized and precise validation studies of human-centered simulations. Formalization and operationalization of aspects of the analysis would further standardize validation, as well as provide subjective measurements for validation. For instance, researchers could be provided with a tool to rate different aspects of the simulation based on DiCoT's principles of distributed cognition. In addition, for validation purposes a standardized method for comparing a DiCoT analysis of the simuland to one of the simulation would be desired - possibly, operationalized as a scoring scheme. 
For SBT, DiCoT can be adapted to distinguish between formative and normative modes of simulation (i.e., training vs. assessment). Whether a simulation is used for training or evaluation can affect which properties of DiCoT and what principles of distributed cognition are applicable. Connecting DiCoT to theoretical principles of learning could further inform how principles of distributed cognition support efficient transfer of training. Moreover, it would be interesting to study and evaluate how cognitive systems adapt as a result of training and internalization of knowledge by cognitive agents. Furthermore, the current DiCoT principles are generally biased towards investigation of temporally and geographically co-located processes. Supporting the analysis of temporally and geographically distributed processes could make DiCoT more applicable for analysis of distributed simulation sessions, such as full-scale emergency simulations using live actors spread over several locations.

\subsubsection{DigEmergo Development}

There are multiple possibilities for the development of the DigEMERGo system, some of which are currently in the works. Two projects have already been initiated that include dynamic patient simulations and constructive stochastic simulation. As mentioned in Paper II and Paper III, the lack of dynamic patient states can be both a limitation and a source of validity issues (especially during assessment), but also a simplification that can aid novice trainees. Dynamic patients can enable new types of training, such as re-triage tasks or sudden changes in patient states to create scenarios that are more challenging. Dynamic patient states can also help create a more accurate and valid assessment of capacity. Moreover, an interesting development of the DigEMERGo system would be to adapt the system for portable mini-training sessions. It can be difficult to plan and perform training sessions for medical personnel, as their workload is generally high and may suddenly increase. Mini-training sessions of about 20 minutes in length that could be performed at the workplace could be used to complement regular training sessions, for instance as refresher courses or as a tool for self-assessment.

The second project, constructive stochastic simulation, refers to using DigEMERGO and the ETS patient bank to predict possible outcomes to major incidents and surge capacity scenarios - so-called "what if' scenarios. For instance, what happens if a fire starts at a crowded nightclub in the city center at 2 am on a Friday night? Stochastic simulations can be used to predict best-case outcomes, identify possible bottlenecks in the EMS system, and to provide information about the effects that changes to the EMS system might have - for instance, whether purchasing a new helicopter would improve outcomes significantly or not.

\subsubsection{A Philosophical Note}

Perhaps my interest in philosophical considerations regarding simulations has been evident throughout this work. Before concluding the thesis, I would like to make a final departure concerning the relationship between simulation and reality. The coupling between technological artifacts and humans seems to get stronger, in both private life and professional settings, as technology gets more advanced. The transhumanist movement (More, 2013) predicts that technology will transform the human condition with sophisticated technologies that will greatly enhance the cognitive and physical abilities of individuals. This outlook has interesting implications for simulation theory. From a distributed cognition perspective, such changes in cognitive capabilities would not be abnormal since individuals already do this, that is, engage in cognitive systems with abilities that supersede those of the naked 
individual ${ }^{36}$. The difference from the present state of affairs is that the technologies suggested by transhumanism will radically change the individual itself (the cognitive agent), making her inseparable from technology (e.g., through nanotechnology and biotechnology).

From a simulation point of view, advancements in technology bring new possibilities as the technology used to change and augment cognitive work in the real world can also be used to create simulated situations which could be, more or less, indistinguishable from reality. For instance, what if we were able to re-play ("mentally simulate") any given situation within the blink of an eye after it has occurred? Or, what if we could simulate situations ahead of time to predict outcomes as they are about to happen? Individuals could, in fact, become their own simulators, meaning that the line between simulated scenarios and reality could be further obscured - if not erased completely. Referring back to the quote by Rosenblueth and Wiener (1945) in Chapter 1, this would create a case of using the same cat as the material model for a cat - but in a simulated and harm-free situation. Let us now consider an example that is grounded in the present time of writing: a digital simulation system for training or assessment of emergency medical management (such as DigEMERGo) could be transformed into a monitoring tool for hospital emergency wards. Vice versa, a digital system that is used to monitor the state of an emergency department could be "reversed" to simulate emergency department scenarios, for use in training, assessment, or prediction. This creates a situation in which the same system and setting could be used in different "modes", rather than using different tools and milieus for training and assessment. Such a practice could engage the real-life cognitive system in the simuland, rather than re-creating it in simulation. Of course, whether such real-life-simulations would be beneficial depends on the purpose of simulation as, for instance, training scenarios can benefit from a simplified task environment.

\subsection{Conclusion}

Human-centered simulations are simulations in which humans are integral to the functionality of the simulation. This thesis suggests that human-centered simulations (e.g., training systems) can be viewed and studied as distributed cognitive systems. Adopting this view allows for re-phrasing of central theoretical constructs in simulation theory using distributed cognition discourse and provides a new approach to the study, design, and evaluation of human-centered simulations. This thesis showed that ethnographical studies using a distributed cognition framework for analysis can be used for validation of simulators to reveal integral mechanisms and functions that pertain to the validity of simulations. Furthermore, the thesis demonstrated that DiCoT can provide a structured analysis that is usable for evaluation as well as for motivation and guiding (re)design of human-centered simulations.

The DigEmergo simulator for training and assessment of emergency medical management was designed, implemented, and evaluated as part of this thesis work. The simulator, which is based on a DiCoT analysis of the Emergo Train System, demonstrated usefulness and application validity - thus indicating the usefulness of distributed cognition as an applied framework for simulator design and evaluation. From a technological research perspective, the new simulator, together with insights gained from evaluating ETS, can be used to improve emergency medical management training and assessment, which in turn can contribute to increased capability to manage emergency medical events. In terms of

\footnotetext{
${ }^{36}$ For those interested in the topic, I would recommend reading Andy Clark's text "Re-Inventing Ourselves"
} (2013) 
the scientific contribution, the results of the thesis contribute to cognitive science, and especially to distributed cognition, by studying a new domain and applying the distributed cognition framework for validation and design of simulators, thereby extending the application of distributed cognition. In doing so, the thesis demonstrated that distributed cognitive systems can be studied within simulated environments and argued that simulation can capture cognitive processes from the wild, thereby opening the door to further research on distributed cognition in simulated environments. Furthermore, the thesis contributes to the simulation field in general and simulation-based training and assessment of emergency medicine management in particular by providing new knowledge and a new perspective on human-centered simulation. 
Chapter 7 


\section{REFERENCES}

Abir, M., Davis, M. M., Sankar, P., Wong, A. C., \& Wang, S. C. (2013). Design of a model to predict surge capacity bottlenecks for burn mass casualties at a large academic medical center. Prehospital and Disaster Medicine, 28(1), 23-32. https://doi.org/10.1017/S1049023X12001513

Adams, F., \& Aizawa, K. (2010). The Bounds of Cognition. Philosophical Psychology, 14(1), 43-64. https://doi.org/10.1002/9781444391718

Adams, F., \& Garrison, R. (2013). The mark of the cognitive. Minds and Machines, 23(2), 339-352. https://doi.org/10.1007/s11023-013-9336-0

Adams, F., \& Garrison, R. (2014). The mark of the cognitive: Reply to Elpidorou. Minds and Machines, 24(2), 213-216. https://doi.org/10.1007/s11023-013-9336-0

Adams, J. A. (1979). On the evaluation of training devices. Human Factors, 21(6), 711-720. https://doi.org/10.1177/001872087912210608

Agar, M. H. (2008). The professional stranger. Cambridge, Massachusetts, USA: Academic Press.

Alessi, S. M. (1988). Fidelity in the Design of Instructional Simulations. Journal of Computer-Based Instruction, 15(2), 40-47.

Andersson, A., D. Carlstrom, E., Ahgren, B., \& M. Berlin, J. (2014). Managing boundaries at the accident scene - a qualitative study of collaboration exercises. International Journal of Emergency Services, 3(1), 77-94. https://doi.org/10.1108/IJES02-2013-0003

Andrews, D. H., Brown, J., Byrnes, J., Chang, J., \& Hartman, R. (1998). Enabling Technology: Analysis of Categories with Potential to Support the Use of Modeling and Simulation in the United States Air Force. (DTIC Report ADA 3518899). Mesa, AZ, USA: Air Force Research Laborotory.

Annett, J. (1996). On knowing how to do things: a theory of motor imagery. Cognitive Brain Research, 3(2), 65-69. https://doi.org/10.1016/0926-6410(95)00030-5

Artino, A. R., Dong, T., DeZee, K. J., Gilliland, W. R., Waechter, D. M., Cruess, D. F., \& Durning, S. J. (2012). Development and Initial Validation of a Survey to Assess Students' Self-Efficacy in Medical School. Military Medicine, 177(9S), 3137. https://doi.org/10.7205/MILMED-D-12-00240

Auf der Heide, E. (2006). The Importance of Evidence-Based Disaster Planning. Annals of Emergency Medicine, 47(1), 34-49. https://doi.org/10.1016/j.annemergmed.2005.05.009

Babbie, E. R. (1992). The practice of social research. Belmont, CA, USA: Wadsworth Pub. Co.

Bandura, A. (1997). Self-efficacy: The exercise of control. London, UK: Macmillan.

Bang, M., \& Timpka, T. (2003). Cognitive tools in medical teamwork: the spatial arrangement of patient records. Methods of Information in Medicine, 42(4), 331-336. https://doi.org/10.1267/METH03040331

Banks, C. M. (2009). What is modelling and simulation? In J. A. Sokolowski \& C. M. Banks (Eds.), Principles of Modeling and Simulation: A Multidisciplinary Approach (pp. 3-24). https://doi.org/10.1002/9780470403563

Banks, C. M. (2010). Introduction to Modeling and Simulation. In J. A. Sokolowski \& C. M. Banks (Eds.), Modeling and Simulation Fundementals: Theoretical Underpinnings and Practical Domains (pp. 1-24). Hoboken, NJ, USA: John Wiley \& Sons, Inc.

Banks, J., Nelson, B. L., Carson, J. S., \& Nicol, D. M. (2014). Discrete-Event System Simulation (Fourth Edi). Pearson. https://doi.org/10.2307/1268124

Barbisch, D. F., \& Koenig, K. L. (2006). Understanding Surge Capacity: Essential Elements. Academic Emergency Medicine, 13(11), 1098-1102. https://doi.org/10.1197/j.aem.2006.06.041

Barker-Plummer, D. (2016). Turing Machines. In E. N. Zalti (Ed.), Stanford Encyclopedia of Philosopby (Winter Ed.). Retrieved from https://plato.stanford.edu/archives/win2016/entries/turing-machine/

Barrows, H. S. (1993). An overview of the uses of standardized patients for teaching and evaluating clinical skills. AAMC. Academic Medicine, 68(6), 443-51. https://doi.org/10.1097/00001888-199306000-00002 
Beaubien, J. M., \& Baker, D. P. (2004). The use of simulation for training teamwork skills in health care: how low can you go? Quality \& Safety in Health Care, 13 Suppl 1, i51-6. https://doi.org/10.1136/qhc.13.suppl_1.i51

Beaudouin-Lafon, M., \& Mackay, W. (2012). Prototyping tools and techniques. In J. A. Jacko (Ed.), The Human-Computer Interaction Handbook (Third Edit, pp. 1081-1104). Boca Raton, FL, USA: CRC Press, Taylor \& Francis Group. https://doi.org/10.1201/9781410615862

Bechtel, W., Abrahamsen, A., \& Graham, G. (1999). The Life of Cognitive Science. In W. Bechtel \& G. Graham (Eds.), A Companion to Cognitive Science (Vol. 13, pp. 1-104). Oxford, UK: Blackwell Publishing Ltd. https://doi.org/10.1111/b.9780631218517.1999.x

Bewley, W. L., \& O’Neil, H. F. (2013). Evaluation of medical simulations. Military Medicine, 178(10 Suppl), 64-75. https://doi.org/10.7205/MILMED-D-13-00255

Beyer, H., \& Holtzblatt, K. (1998). Contextual design: Defining customer-centered systems. San Francisco: Morgan Kaufmann.

Blackwell, T. H., \& Kaufman, J. S. (2002). Response time effectiveness: Comparison of response time and survival in an urban emergency medical services system. Academic Emergency Medicine, 9(4), 288-295. https://doi.org/10.1111/j.15532712.2002.tb01321.x

Blandford, A., Berndt, E., Catchpole, K., Furniss, D., Mayer, A., Mentis, H. M., ... Randell, R. (2015). Strategies for conducting situated studies of technology use in hospitals. Cognition, Technology \& Work, 17(4), 1-14. https://doi.org/10.1007/s10111-014-0318-7

Blandford, A., \& Furniss, D. (2006). DiCoT: a methodology for applying distributed cognition to the design of teamworking systems. In S. Gilroy \& M. Harrison (Eds.), 12th International Workshop on Design, Specification and Verification of Interactive Systems (pp. 26-38). Springer-Verlag Berlin.

Blandford, A., \& Wong, B. W. (2004). Situation awareness in emergency medical dispatch. International Journal of HumanComputer Studies, 61(4), 421-452.

Bloomberg, J., \& Burrell, M. (2012). An Ethnographic Approach To Design. In J. A. Jacko (Ed.), The Human-Computer Interaction Handbook (Third Edit, pp. 1025-1052). Boca Raton, FL, USA: CRC Press, Taylor \& Francis Group.

Bohr, N. (1913). On the constitution of atoms and molecules. Philosophical Magazine Series 6, 26(151), 1-25. https://doi.org/10.1007/978-3-319-14316-3_2

Bolling, R., Ehrlin, Y., Forsberg, R., Rüter, A., Soest, V., Vikström, T., ... Brändström, H. (2007). KAMEDO Report 90: terrorist attacks in Madrid, Spain, 2004. Prehospital and Disaster Medicine, 22(3), 252-7.

Boocock, S. S. (1972). Validity-Testing of an Intergenerational Relations Game. Simulation \& Games, 3(1), 29-40. https://doi.org/10.1177/104687817200300103

Box, G. E. P. (1976). Science and Statistics. Journal of the American Statistical Association, 71(356), 791-799. https://doi.org/10.1641/B570910

Brannan, J. D., White, A., \& Bezanson, J. L. (2008). Simulator effects on cognitive skills and confidence levels. The Journal of Nursing Education, 47(11), 495-500.

Bratzke, E., Downs, J. B., \& Smith, R. A. (1998). A Comprehensive Anesthesia Simulation Environment: Re-creating the Operation Room for Research and. Anesthesiology, 89(2), 334-340. https://doi.org/10.1097/00000542-19980800000008

Britt, R. C., Scerbo, M. W., Montano, M., Kennedy, R. A., Prytz, E. G., \& Stefanidis, D. (2015). Transfer from Fundamentals of Laparoscopic Surgery to cadavers results in substantial increase in mental workload. Surgery, 158(5), 1428-1433. https://doi.org/10.1016/j.surg.2015.03.032

Brooks, H. (1994). The relationship between science and technology. Research Policy, 23(5), 477-486. https://doi.org/10.1016/0048-7333(94)01001-3

Caird, J. K. (1996). Persistent issues in the application of virtual environment systems to training. In Proceedings Third Annual Symposium on Human Interaction with Complex Systems. HICS'96 (pp. 124-132). Los Alamitos, CA, USA: IEEE Computer Society Press. https://doi.org/10.1109/HUICS.1996.549502

Campbell, B. D., Mete, H. O., Furness, T., Weghorst, S., \& Zabinsky, Z. (2008). Emergency Response Planning and Training through Interactive Simulation and Visualization with Decision Support. In 2008 IEEE Conference on Technologies for Homeland Security (pp. 176-180). Waltham, MA, USA: IEEE. https://doi.org/10.1109/THS.2008.4534445

Campbell, J. P. (1990). Modeling the Performance Prediction Problem in Industrial and Organizational Psychology. In M. D. Dunnette \& L. M. Hough (Eds.), Handbook of industrial and organizational psychology (Vol. 1, pp. 687-732). Palo Alto, CA, US: Consulting Psychologists Press. 
Candela, L. (2012). From Teaching to Learning: Theoretical Foundations. In D. Billings \& J. Halstead (Eds.), Teaching in nursing: A guide for faculty (pp. 202-243). St Louis: Elsevier.

Cannon, H. M., \& Burns, A. C. (1999). A Framework For Assessing The Competencies Reflected In Simulation Performance. Developments in Business Simulation \& Experiential Exercises, 26, 40-44.

Cant, R. P., \& Cooper, S. J. (2010). Simulation-based learning in nurse education: systematic review. Journal of Advanced Nursing, 66(1), 3-15. https://doi.org/10.1111/j.1365-2648.2009.05240.x

Carroll, J. M. (2013). Human Computer Interaction - brief intro. In M. Soegaard \& R. F. Dam (Eds.), The Encyclopedia of HumanComputer Interaction (2nd Editio, pp. 1-69). The Interaction Design Foundation. https://doi.org/10.1177/0011392199047004007

Carroll, J. M., \& Rosson, M. B. (1992). Getting around the task-artifact cycle: how to make claims and design by scenario. ACM Transactions on Information Systems, 10(2), 181-212. https://doi.org/10.1145/146802.146834

Carsten, O., \& Jamson, H. (2011). Driving simulators as research tools in traffic psychology. In B. Porter (Ed.), Handbook of Traffic Psychology (pp. 87-96). Cambridge, MA, USA: Academic Press. https://doi.org/10.1016/B978-0-12-3819840.10007-4

Carter, F. J., Schijven, M. P., Aggarwal, R., Grantcharov, T., Francis, N. K., Hanna, G. B., \& Jakimowicz, J. J. (2006). Consensus guidelines for validation of virtual reality surgical simulators. Simulation in Healthcare, 1(3), 171-179. https://doi.org/10.1007/s00464-005-0384-2

Chandrasekharan, S., \& Nersessian, N. J. (2015). Building Cognition: The Construction of Computational Representations for Scientific Discovery. Cognitive Science, 39(8), 1727-1763. https://doi.org/10.1111/cogs.12203

Chang, T. P., Kwan, K. Y., Liberman, D., Song, E., Dao, E. H., Chung, D., ... Festekjian, A. (2015). Introducing Teamwork Challenges in Simulation Using Game Cards. Simulation in Healthcare, 10(4), $223-6$. https://doi.org/10.1097/SIH.0000000000000089

Chi, C.-H. H., Chao, W.-H. H., Chuang, C.-C. C., Tsai, M.-C. C., Tsai, L.-M. M., Shemer, J., ... Bass, E. B. (2001). Emergency medical technicians' disaster training by tabletop exercise. American Journal of Emergency Medicine, 19(5), 433-436. https://doi.org/10.1053/ajem.2001.24467

Cicero, M. X., Walsh, B., Solad, Y., Whitfill, T., Paesano, G., Kim, K., ... Cone, D. C. (2015). Do You See What I See? Insights from Using Google Glass for Disaster Telemedicine Triage. Prehospital and Disaster Medicine, 30(February), 1-5. https://doi.org/10.1017/S1049023X1400140X

Clark, A. (1998). Embodied, situated, and distributed cognition. In W. Bechtel \& G. Graham (Eds.), A companion to cognitive science (pp. 506-517). Hoboken, NJ, USA: Blackwell Publishing Ltd. https://doi.org/10.1111/b.9780631218517.1999.00042.x

Clark, A. (2007). Re-inventing ourselves: the plasticity of embodiment, sensing, and mind. The Journal of Medicine and Philosophy, 32(3), 263-82. https://doi.org/10.1080/03605310701397024

Clark, A. (2013). Re-Inventing Ourselves. In The Transhumanist Reader (pp. 113-127). Oxford: John Wiley \& Sons. https://doi.org/10.1002/9781118555927.ch11

Cohen, D., Sevdalis, N., Taylor, D., Kerr, K., Heys, M., Willett, K., ... Darzi, A. (2013). Emergency preparedness in the 21st century: training and preparation modules in virtual environments. Resuscitation, 84(1), 78-84. https://doi.org/10.1016/j.resuscitation.2012.05.014

Cohen, T., Blatter, B., Almeida, C., Shortliffe, E., \& Patel, V. (2006). A cognitive blueprint of collaboration in context: distributed cognition in the psychiatric emergency department. Artificial Intelligence in Medicine, 37(2), 73-83. https://doi.org/10.1016/j.artmed.2006.03.009

Conway, R. W. (1963). Some Tactical Problems in Digital Simulation. Management Science, 10(1), 47-61. https://doi.org/10.1287/mnsc.10.1.47

Cooper, J. B., \& Taqueti, V. R. (2008). A brief history of the development of mannequin simulators for clinical education and training. Postgraduate Medical Journal, 84(997), 563-570. https://doi.org/10.1136/qshc.2004.009886

Crandall, B., Klein, G., \& Hoffman, R. (2006). Working Minds: a Practitioner's Guide to Cognitive Task. Analysis. Cambridge, Massachusetts, USA: The MIT Press. https://doi.org/978-0262532815

Crookall, D. (2010). Serious Games, Debriefing, and Simulation/Gaming as a Discipline. Simulation \& Gaming, 41(6), 898920. https://doi.org/10.1177/1046878110390784

Crookall, D., Oxford, R., \& Saunders, D. (1987). Towards a Reconceptualization of Simulation: From Representation to Reality. Simulation/ Games for Learning, 17(4), 147-71. 
Dahl, Y., Alsos, O. A., \& Svanæs, D. (2010). Fidelity considerations for simulation-based usability assessments of mobile ICT for hospitals. International Journal of Human-Computer Interaction, 26(5), 445-476. https://doi.org/10.1080/10447311003719938

Dahlstrom, N., Dekker, S., Van Winsen, R., \& Nyce, J. (2009). Fidelity and validity of simulator training. Theoretical Issues in Ergonomics Science, 10(4), 305-314. https://doi.org/10.1080/14639220802368864

Daniels, K., \& Auguste, T. (2013). Moving forward in patient safety: multidisciplinary team training. Seminars in Perinatology, 37(3), 146-50. https://doi.org/10.1053/j.semperi.2013.02.004

Davis, F. D. (1989). Perceived Usefulness, Perceived Ease Of Use, And User Acceptance. MIS Quarterly, 13(3), 319-339. https://doi.org/10.2307/249008

Dawe, S. R., Pena, G. N., Windsor, J. A., Broeders, J. A. J. L., Cregan, P. C., Hewett, P. J., \& Maddern, G. J. (2014). Systematic review of skills transfer after surgical simulation-based training. British Journal of Surgery, 101(9), 1063-1076. https://doi.org/10.1002/bjs. 9482

Decker, S., Sportsman, S., Puetz, L., \& Billings, L. (2008). The Evolution of Simulation and Its Contribution to Competency. The Journal of Continuing Education in Nursing, 39(2), 74-80. https://doi.org/10.3928/00220124-20080201-06

Dieckmann, P., Gaba, D. M., \& Rall, M. (2007). Deepening the theoretical foundations of patient simulation as social practice. Simulation in Healthcare, 2(3), 183-93. https://doi.org/10.1097/SIH.0b013e3180f637f5

DoD M\&S Glossary. (2010). Simulation. Glossary of Modeling and Simulation (M\& S) Terms. https://doi.org/DoD 5000.59-M

Drews, F. A., \& Bakdash, J. Z. (2013). Simulation Training in Health Care. Reviews of Human Factors and Ergonomics, 8(1), 191234. https://doi.org/10.1177/1557234X13492977.

Dukes, R. L., \& Waller, S. J. (1976). Review Essay: Toward a General Evaluation Model for Simulation Games: GEM. Simulation \& Gaming, 7(1), 75-88. https://doi.org/10.1177/104687817600700106

Elpidorou, A. (2014). Reasoning about the mark of the cognitive: A response to Adams and Garrison. Minds and Machines, 24(2), 201-211. https://doi.org/10.1007/s11023-013-9319-1

Eppich, W., Howard, V., Vozenilek, J., \& Curran, I. (2011). Simulation-based team training in healthcare. Simulation in Healthcare, 6, S14-19. https://doi.org/10.1097/SIH.0b013e318229f550

Epstein, L. A. (2004). The Technology Challenge: Lawyers Have Finally Entered the Race But Will Ethical Hurdles Slow the Pace. Nova Law Review, 28(3), 721-743.

Fanning, R. M., \& Gaba, D. M. (2007). The role of debriefing in simulation-based learning. Simulation in Healthcare, 2(2), 115125. https://doi.org/10.1097/SIH.0b013e3180315539

Feinstein, A. H., \& Cannon, H. M. (2001). Fidelity, verifiability, and validity of simulation: Constructs for evaluation. Simulation \& Gaming, 33(4), 57-67.

Feinstein, A. H., \& Cannon, H. M. (2002). Constructs of Simulation Evaluation. Simulation \& Gaming, 33(4), 425-440. https://doi.org/10.1177/1046878102238606

Feinstein, A. H., \& Cannon, H. M. (2003). A hermeneutical approach to external validation of simulation models. Simulation \& Gaming, 34(2), 186-197. https://doi.org/10.1177/1046878103252885

Feinstein, A. H., Mann, S., \& Corsun, D. L. (2002). Charting the experiential territory: Clarifying definitions and uses of computer simulation, games, and role play. Journal of Management Development, 21(10), 732-744. https://doi.org/10.1108/02621710210448011

Fields, B., \& Wright, P. (2000). Analysing human-computer interaction as distributed cognition. Human-Computer Interaction Journal, 15(1), 1-31. https://doi.org/10.1207/S15327051HCI1501_01

Forsblad, M. (2016). Distributed cognition in bome environments (Doctoral Dissertation). Linköping Studies in Arts and Science, Linköping University. Retrieved from https://doi.org/10.3384/diss.diva-131420

Franc, J. M., Ingrassia, P. L., Verde, M., Colombo, D., \& Della Corte, F. (2014). A simple graphical method for quantification of disaster management surge capacity using computer simulation and process-control tools. Prehospital and Disaster Medicine, 30(1), 1-7. https://doi.org/10.1017/S1049023X1400123X

Furniss, D. (2008). Beyond problem identification: valuing methods in a "system usability practice" (Doctoral Dissertation). University College London. Retrieved from http://eprints.ucl.ac.uk/14221/

Furniss, D., \& Blandford, A. (2006). Understanding emergency medical dispatch in terms of distributed cognition: a case study. Ergonomics, 49(12-13), 1174-203. https://doi.org/10.1080/00140130600612663 
Furniss, D., \& Blandford, A. (2010). DiCoT Modeling: From Analysis to Design. In Proceedings of CHI 2010 Workshop Bridging the Gap: Moving from Contextual Analysis to Design (pp. 10-15). Atlanta, GA, USA. Retrieved from http://www.cs.ucl.ac.uk/staff/d.furniss/Documents/DiCoT analysis n design.pdf

Furniss, D., Masci, P., Curzon, P., Mayer, A., \& Blandford, A. (2015). Exploring medical device design and use through layers of Distributed Cognition: How a glucometer is coupled with its context. Journal of Biomedical Informatics, 53, 330-341. https://doi.org/10.1016/j.jbi.2014.12.006

Gaba, D. M. (2004). The future vision of simulation in health care. Quality \& Safety in Health Care, 13(Suppl 1), i2-10. https://doi.org/10.1136/qhc.13.suppl_1.i2

Gallagher, A. G., \& Satava, R. M. (2002). Virtual reality as a metric for the assessment of laparoscopic psychomotor skills: Learning curves and reliability measures. Surgical Endoscopy and Other Interventional Techniques, 16(12), 1746-1752. https://doi.org/10.1007/s00464-001-8215-6

Garbis, C. (2002). The cognitive use of artifacts in cooperative process management: Rescue management and underground line control (Doctoral Dissertation). Linköping Studies in Arts and Science, Linköping University. Retrieved from http://urn.kb.se/resolve?urn=urn:nbn:se:liu:diva-33962

Gebbie, K. M., \& Qureshi, K. (2002). Emergency and disaster preparedness: core competencies for nurses. The American Journal of Nursing, 102(1), 46-51.

Gillen, J., Ferguson, R., Peachey, A., \& Twining, P. (2012). Distributed cognition in a virtual world. Language and Education, 26(2), 151-167. https://doi.org/10.1080/09500782.2011.642881

Gillett, B., Peckler, B., Sinert, R., Onkst, C., Nabors, S., Issley, S., .. Arquilla, B. (2008). Simulation in a disaster drill: Comparison of high-fidelity simulators versus trained actors. Academic Emergency Medicine, 15(11), 1144-1151. https://doi.org/10.1111/j.1553-2712.2008.00198.x

Goodwin, C. (1994). Professional vision. American Anthropologist, 96(3), 606-633.

Graham, G. (2017). Behaviorism. In E. N. Zalta (Ed.), The Stanford Encyclopedia of Pbilosophy (Spring Ed.). Retrieved from https://plato.stanford.edu/archives/spr2017/entries/behaviorism/

Granlund, R. (2017). C3Fire. Retrieved December 5, 2017, from http://www.c3learninglabs.com/mw/index.php/C3Fire:Main

Gray, W. D. (2002). Simulated Task Environments: The Role of High-Fidelity Simulations, Scaled Worlds, Synthetic Environments, and Laboratory Tasks in Basic and Applied Cognitive Research. Cognitive Science Quarterly, 2(2), 205227.

Grenvik, A., \& Schaefer, J. (2004). From Resusci-Anne to Sim-Man: the evolution of simulators in medicine. Critical Care Medicine, 32(2 Suppl), S56-57. https://doi.org/10.1097/00003246-200402001-00010

Grier, R. A. (2015). How high is high? A meta-analysis of NASA-TLX global workload scores. In Proceedings of the Human Factors and Ergonomics Society (pp. 1727-1731). Los Angeles, CA, USA: Sage Publications. https://doi.org/10.1177/1541931215591373

Grossman, R., \& Salas, E. (2011). The transfer of training: what really matters. International Journal of Training and Development, 15(2), 103-120. https://doi.org/10.1111/j.1468-2419.2011.00373.x

Grune-Yanoff, T., \& Weirich, P. (2010). The Philosophy and Epistemology of Simulation: A Review. Simulation \& Gaming, 41(1), 20-50. https://doi.org/10.1177/1046878109353470

Gryth, D., Rådestad, M., Nilsson, H., Nerf, O., Svensson, L., Castrén, M., \& Rüter, A. (2010). Evaluation of medical command and control using performance indicators in a full-scale, major aircraft accident exercise. Prehosp Disaster Med, 25(2), $118-124$.

Halverson, C. A. (1994). Distributed Cognition as a Theoretical Framework for HCI: Don't Throw the Baby Out With the Bathwater The Importance of the Cursor in Air Traffic Control. (Tech Report 9403). San Diego, CA, USA: Department of Cognitive Science, University of California.

Halverson, C. A. (2002). Activity theory and distributed cognition: Or what does CSCW need to DO with theories? Computer Supported Cooperative Work. (CSCW), 11(1), 243-267.

Hamstra, S. J., Brydges, R., Hatala, R., Zendejas, B., \& Cook, D. A. (2014). Reconsidering fidelity in simulation-based training. Academic Medicine, 89(3), 387-92. https://doi.org/10.1097/ACM.0000000000000130

Hancock, P., Vincenzi, D., Wise, J., \& Mouloua, M. (2010). Human factors in simulation and training. Boca Raton, FL, USA: CRC Press, Inc.

Hardy, D. J., \& Parasuraman, R. (1997). Cognition and Flight Performance in Older Pilots. Journal of Experimental Psychology: Applied, 3(4), 313-348. https://doi.org/10.1037/1076-898X.3.4.313 
Hart, S. G., \& Staveland, L. E. (1988). Development of NASA-TLX (Task Load Index): Results of Empirical and Theoretical Research. Advances in Psychology, 52(C), 139-183. https://doi.org/10.1016/S0166-4115(08)62386-9

Hartmann, S. (1996). The World as a Process. In Modelling and Simulation in the Social Sciences from the Philosophy of Science Point of View (pp. 77-100). Dordrecht: Springer Netherlands. https://doi.org/10.1007/978-94-015-8686-3_5

Hick, J. L., Barbera, J. A., \& Kelen, G. D. (2000). Refining surge capacity: conventional, contingency, and crisis capacity. Disaster Medicine and Public Health Preparedness, 3(1), 59-67. https://doi.org/10.5580/1f17

Hirshberg, A., Frykberg, E. R., Mattox, K. L., \& Stein, M. (2010). Triage and trauma workload in mass casualty: a computer model. The Journal of Trauma, 69(5), 1074-81-2. https://doi.org/10.1097/TA.0b013e3181e50624

Hochmitz, I., \& Yuviler-Gavish, N. (2011). Physical Fidelity Versus Cognitive Fidelity Training in Procedural Skills Acquisition. Human Factors, 53(5), 489-501. https://doi.org/10.1177/0018720811412777

Hoffman, R. R., \& Deffenbacher, K. A. (1993). An analysis of the relations between basic and applied psychology. Ecological Psychology, 5(4), 315-352. https://doi.org/10.1207/s15326969eco0504_3

Hollan, J., Hutchins, E., \& Kirsh, D. (2000). Distributed cognition: toward a new foundation for human-computer interaction research. ACM Transactions on Computer-Human Interaction (TOCHI), 7(2), 174-196.

Hollnagel, E. (2011). Simulator Studies: The Next Best Thing? In A. B. Skjerve \& A. Bye (Eds.), Simulator-based Human Factors Studies Across 25 Years: The History of the Halden Man-Machine Laboratory (pp. 75-90). London: Springer. https://doi.org/10.1007/978-0-85729-003-8

Hollnagel, E., \& Woods, D. (2005). Joint cognitive systems: Foundations of cognitive systems engineering. Boca Raton, FL, USA: CRC Press.

Hontvedt, M. (2015). Professional vision in simulated environments - Examining professional maritime pilots' performance of work tasks in a full-mission ship simulator. Learning, Culture and Social Interaction, 7, 71-84. https://doi.org/10.1016/j.lcsi.2015.07.003

Horst, S. (1999). Symbols and Computation A Critique of the Computational Theory of Mind. Minds and Macbines, 9, 347381.

Hsu, E. B., Jenckes, M. W., Catlett, C. L., Robinson, K. A., Feuerstein, C., Cosgrove, S. E., ... Bass, E. B. (2004). Effectiveness of hospital staff mass-casualty incident training methods: a systematic literature review. Prehospital and Disaster Medicine, 19(3), 191-199.

Humphreys, P. (2004). Extending Ourselves: Computational Science, Empiricism, and Scientific Method. Oxford, UK: Oxford University Press.

Hutchins, E. (1995a). Cognition in the Wild (Vol. 19). MIT press: Cambridge, MA, USA. Retrieved from https://mitpress.mit.edu/books/cognition-wild

Hutchins, E. (1995b). How a Cockpit Remembers its Speeds. Cognitive Science, 19(3), 265-288.

Jacko, J. A. (2012). The Human-Computer Interaction Handbook: Fundamentals, Evolving Technologies, and Emerging Applications. The buman-computer interaction bandbook (Third Edit). Boca Raton, FL, USA: CRC Press, Taylor \& Francis Group.

Jones, R. E. T., McNeese, M. D., Connors, E. S., Jefferson, T., \& Hall, D. L. (2004). A Distributed Cognition Simulation Involving Homeland Security and Defense: The Development of Neocities. Proceedings of the Human Factors and Ergonomics Society Annual Meeting, 48(3), 631-634. https://doi.org/10.1177/154193120404800376

Juffermans, J., \& Bierens, J. J. L. M. (2010). Recurrent medical response problems during five recent disasters in the Netherlands. Prehospital and Disaster Medicine, 25(2), 127-136.

Kaji, A. H., Koenig, K. L., \& Lewis, R. J. (2007). Current Hospital Disaster Preparedness. JAMA, 298(18), 2188. https://doi.org/10.1001/jama.298.18.2188

Ker, J., Mole, L., \& Bradley, P. (2003). Early introduction to interprofessional learning: A simulated ward environment. Medical Education, 37(3), 248-255. https://doi.org/10.1046/j.1365-2923.2003.01439.x

Kibbee, J. M. (1961). Model building for management games. In A. Newgarden (Ed.), Simulation and gaming: A symposium (pp. 8-15). New york, NY, USA: American Management Association.

Kirsh, D. (1995). The intelligent use of space. Artificial Intelligence, 73(1-2), 31-68.

Kirsh, D. (2010). Thinking with external representations. AI and Society, 25(4), 441-454. https://doi.org/10.1007/s00146-0100272-8

Kirsh, D. (2013). Embodied cognition and the magical future of interaction design. ACM Transactions on Computer-Human Interaction, 20(1), 3:1-3:30. https://doi.org/10.1145/2442106.2442109 
Kirsh, D., \& Maglio, P. (1994). On Distinguishing Epistemic from Pragmatic Action. Cognitive Science, 18(4), 513-549. https://doi.org/10.1207/s15516709 $\operatorname{cog} 1804 \_1$

Klein, E. E. (2005). Philosophical foundations of computer simulation validation. Simulation \& Gaming, 36(3), 303-329. https://doi.org/10.1177/1046878104273437

Kleindorfer, G. B., O’Neill, L., \& Ganeshan, R. (1998). Validation in Simulation: Various Positions in the Philosophy of Science. Management Science, 44(8), 1087-1099. https://doi.org/10.1287/mnsc.44.8.1087

Kneebone, R. (2003). Simulation in surgical training: educational issues and practical implications. Medical Education, 37(3), 267-77.

Kneebone, R., \& Baillie, S. (2008). Contextualized Simulation and Procedural Skills: A View from Medical Education. Journal of Veterinary Medical Education, 35(4), 595-598. https://doi.org/10.3138/jvme.35.4.595

Knight, J. F., Carley, S., Tregunna, B., Jarvis, S., Smithies, R., de Freitas, S., ... Mackway-Jones, K. (2010). Serious gaming technology in major incident triage training: a pragmatic controlled trial. Resuscitation, 81(9), 1175-9. https://doi.org/10.1016/j.resuscitation.2010.03.042

Kolb, D. A. (1984). Experiential Learning: Experience as The Source of Learning and Development. Prentice Hall, Inc., (1984), 20-38. https://doi.org/10.1016/B978-0-7506-7223-8.50017-4

Kozlowski, S., \& DeShon, R. (2004). A Psychological Fidelity Approach to Simulation-Based Training: Theory, Research, and Principles. In S. G. Schiflett, L. R. Elliot, E. Salas, \& M. D. Coovert (Eds.), Scaled Worlds: Development, Validation, and applications (pp. 75-99). Farnham, Surrey, Storbritannien: Ashgate Pub Co.

L3-Technologies. (2017). Link Trainer History. Retrieved April 14, 2017, from https://www.link.com/about/pages/history.aspx

Leardal Medical. (n.d.). The Story of Resusci Anne and the beginnings of Modern CPR. Retrieved June 27, 2017, from http://www.laerdal.com/gb/doc/2738/The-Story-of-Resusci-Anne-and-the-beginnings-of-Modern-CPR

Legemaate, G. A., Burkle Jr., F. M., \& Bierens, J. J. (2012). The evaluation of research methods during disaster exercises: Applicability for improving disaster health management. Prehospital and Disaster Medicine, 27(1), 18-26. https://doi.org/10.1017/S1049023X11006789

Lennquist, S. (2005). Education and training in disaster medicine. Scandinavian Journal of Surgery, 94(4), 300-10.

Lennquist, S. (2009). Katastrofmedicin. Stockholm, Sweden: Liber AB.

Lennquist, S. (2012). Medical Response to Major Incidents and Disasters. Berlin, Germany: Springer-Verlag. https://doi.org/10.1007/978-3-642-21895-8

Liu, D., Macchiarella, N., \& Vincenzi, D. (2008). Simulation Fidelity. In P. A. Hancock, D. Vincenzi, J. A. Wise, \& M. Mouloua (Eds.), Human Factors in Simulation and Training (pp. 61-73). Boca Raton, FL, USA: CRC Press. https://doi.org/10.1201/9781420072846.ch4

Lopreiato, J., Downing, D., Hargreaves, O., Carmack, J., Coerver, D., Decker, S., .. Martinez, A. (2016). Healthcare Simulation Dictionary. Rockville, MD, USA: Agency for Healthcare Research and Quality. Retrieved from http://www.ahrq.gov/professionals/quality-patient-safety/patient-safety-resources/research/simulationdictionary/index.html

Löve, J., Moore, C. D., \& Hensing, G. (2012). Validation of the Swedish translation of the general self-efficacy scale. Quality of Life Research, 21(7), 1249-1253. https://doi.org/10.1007/s11136-011-0030-5

Maran, N. J., \& Glavin, R. J. (2003). Low- to high-fidelity simulation - a continuum of medical education? Medical Education, $37(1), 22-28$.

Marr, D. (1982). Vision: A Computational Investigation into the Human Representation and Processing of Visual Information. Cambridge, MA, USA: MIT press.

Mathis, K. L., \& Wiegmann, D. A. (2007). Construct validation of a laparoscopic surgical simulator. Simulation in Healthcare, 2(3), 178-82. https://doi.org/10.1097/SIH.0b013e318137aba1

McDougall, E. M. (2007). Validation of surgical simulators. Journal of Endourology, 21(3), $244-247$. https://doi.org/10.1089/end.2007.9985

Meakim, C. H., Boese, T., Decker, S., Franklin, A., Gloe, D., Lioce, L., ... Borum, J. (2013). Standards of Best Practice: Simulation Standard I: Terminology. Clinical Simulation in Nursing, 9(6), 3-11. https://doi.org/10.1016/j.ecns.2015.03.005

Mihram, G. A. (1972). Some Practical Aspects of the Verification and Validation of Simulation Models. Operational Research Quarterly (1970-1977), 23(1), 17. https://doi.org/10.2307/3008511 
Miller, G. (2003). The cognitive revolution: a historical perspective. Trends in Cognitive Sciences, 7(3), 141-144. https://doi.org/10.1016/S1364-6613(03)00029-9

Miller, R. B. (1954). Psychological Considerations In The Design Of Training Equipment. Wright-Patterson Air Force Base, Ohio, USA: Wright Air Development Center, Air Research and Development Command, United States Air Force.

Moore, L. (1999). Measuring quality and effectiveness of prehospital ems. Prehospital Emergency Care, 3(4), 325-331. https://doi.org/10.1080/10903129908958963

More, M. (2013). The Philosophy of Transhumanism. In N. Vita-More \& M. More (Eds.), The Transhumanist Reader: Classical and Contemporary Essays on the Science, Technology, and Pbilosophy of the Human Future (pp. 3-17). John Wiley \& Sons, Inc.

Morris, C. D., Bransford, J. D., \& Franks, J. J. (1977). Levels of processing versus transfer appropriate processing. Journal of Verbal Learning and Verbal Behavior, 16(5), 519-533. https://doi.org/10.1016/S0022-5371(77)80016-9

Naikar, N., Hopcroft, R., \& Moylan, A. (2005). Work domain analysis: Theoretical concepts and methodology. Defence Science and Technology Report (Vol. $\quad 1) . \quad$ Retrieved from http://oai.dtic.mil/oai/oai?verb=getRecord\&amp;metadataPrefix=html\&amp;identifier=ADA449707

Narayanan, S., \& Kidambi, P. (2011). Interactive Simulations: History, Features, and Trends. In L. Rothrock \& S. Narayanan (Eds.), Human-in-the-Loop Simulations (pp. 1-15). London, UK: Springer-Verlag London Limited.

Neisser, U. (1967). Cognitive psychology. New york, NY, USA: Appleton-Century-Crofts.

Nestel, Groom, J., Eikeland-Husebø, S., \& O’Donnell, J. M. (2011). Simulation for learning and teaching procedural skills: The state of the science. Simulation in Healthcare, 6(7), S10-S13.

Newell, A., \& Simon, H. A. (1972). Human problem solving. Upper Saddle River, NJ, USA: Prentice-Hall.

Newell, A., Simon, H. a., Newel, A., \& Simon, H. a. (1976). Completer Science as Empirical Inquiry: Symbols and Search. Communications of the ACM, 19(3), 113-126. https://doi.org/10.1145/360018.360022

Nilsson, H. (2013). Demand for Rapid and Accurate Regional Medical Response at Major Incidents (Doctoral Dissertation). Faculty of Health Sciences, Linköping University. Retrieved from http://liu.divaportal.org/smash/record.jsf?pid=diva2:579248

Nilsson, H., Jonson, C.-O., Vikström, T., Bengtsson, E., Thorfinn, J., Huss, F., ... Sjöberg, F. (2013). Simulation-assisted burn disaster planning. Burns, 39(6), 1122-1130.

Nilsson, H., Vikström, T., \& Rüter, A. (2010). Quality control in disaster medicine training--initial regional medical command and control as an example. American Journal of Disaster Medicine, 5(1), 35-40.

Nordin, I. (2017). Using Knowledge: On the Rationality of Science, Technology, and Medicine. Lanham, MD, USA: Lexington Books.

Norman, D. A. (1994). Things that make us smart. Boston, MA, USA: Addison-Wesley Longman Publishing Co., Inc.

Norris, D. R. (1986). External validity of business games. Developments in Business Simulation \& Experiential Exercises, 13(4), 126129. https://doi.org/10.1177/0037550086174003

Okasha, S. (2002). Philosophy of Science: A very short introduction. New york, NY, USA: Oxford University Press.

Okuda, Y., \& Bryson, E. (2009). The utility of simulation in medical education: what is the evidence? The Mount Sinai Journal of Medicine, 76(4), 330-43

Ören, T. (2005). Toward the Body of Knowledge of Modeling and Simulation. In Interservice/Industry Training, Simulation, and Educational Conference (pp. 1-19). Orlando, FL, USA.

Ören, T. (2011a). A Basis for a Modeling and Simulation Body of Knowledge Index : Professionalism, Stakeholders, Big Picture, and Other BoKs. SCS M\&S Magazine, 1, 40-48.

Ören, T. (2011b). A Critical Review of Definitions and About 400 Types of Modeling and Simulation. SCS M\&s Magazine, 2(3), 142-151.

Ören, T. (2011c). The Many Facets of Simulation through a Collection of about 100 Definitions. SCS M\&S Magazine, 2(2), 82-92.

Ören, T., \& Zeigler, B. P. (2012). System theoretic foundations of modeling and simulation: a historic perspective and the legacy of A Wayne Wymore. Simulation: Transactions of SCS, 88(9), 1033-1046. https://doi.org/10.1177/0037549712450360

Pace, D. K. (2004). Modeling and simulation verification and validation challenges. Johns Hopkins APL Technical Digest (Applied Physics Laboratory), 25(2), 163-172.

Padilla, J. J., Diallo, S. Y., \& Tolk, A. (2011). Do We Need M\&S Science? SCS M\&S Magazine, 4, 161-166. 
Pattillo, M. (2006). Teaching Disaster Nursing Response Using Simulations. Clinical Simulation in Nursing, 2(2), e49-e51. https://doi.org/10.1016/j.ecns.2009.05.020

Pegden, C. D., Sadowski, R. P., \& Shannon, R. E. (1995). Introduction to Simulation Using SIMAN. New york, NY, USA: McGraw-Hill, Inc. Retrieved from http://dl.acm.org/citation.cfm?id=550225

Perry, M. J. (1997). Distributed cognition and computer supported collaborative design: the organisation of work in construction engineering (Doctoral Dissertation). Brunel University.

Perry, M. J. (2003). Distributed cognition. In J. M. Carroll (Ed.), HCI Models, Theories, and Frameworks: Toward a Multidisciplinary Science (pp. 212-223). San Francisco, CA, USA: Morgan Kauffman Publishers.

Peters, V., Vissers, G., \& Heijne, G. (1998). The Validity of Games. Simulation \& Gaming, 29(1), 20-30. https://doi.org/10.1177/1046878198291003

Petty, M. (2010). Verification, Validation, and Accreditation. In J. A. Sokolowski \& C. M. Banks (Eds.), Modeling and Simulation Fundementals: Theoretical Underpinnings and Practical Domains. Hoboken, NJ: John Wiley \& Sons, Inc.

Pool, R. (1992). The Third Branch of Science Debuts. Science, 256(5053), 44-47. https://doi.org/10.1126/science.256.5053.44

Princeton University. (2017a). Simulate. Retrieved July 6, 2017, from http://wordnet-rdf.princeton.edu/wn31/simulate-v

Princeton University. (2017b). Simulation. Retrieved June 7, 2017, from http://wordnetweb.princeton.edu/perl/webwn?s=simulation

Prytz, E. G., Rybing, J., Jonson, C.-O., Prytz, PhD, E. G., Rybing, MSc, J., \& Jonson, PhD, C.-O. (2016). Workload differences across command levels and emergency response organizations during a major joint training exercise. Journal of Emergency Management, 14(4), 289-97. https://doi.org/10.5055/jem.2016.0294

Rådestad, M., Nilsson, H., Castrén, M., Svensson, L., Rüter, A., \& Gryth, D. (2012). Combining Performance and Outcome Indicators can be used in a Standardized way for creating Lessons Learned in Disaster Response Training: an Observational study of two of Multidisciplinary, Full-scale Major Aircraft Exercises. Scandinavian Journal of Trauma, Resuscitation and Emergency Medicine, 20(58). https://doi.org/10.1186/1757-7241-20-58

Rajkomar, A., \& Blandford, A. (2012). Understanding infusion administration in the ICU through Distributed Cognition. Journal of Biomedical Informatics, 45(3), 580-590. https://doi.org/10.1016/j.jbi.2012.02.003

Rajkomar, A., Blandford, A., \& Mayer, A. (2013). Coping with complexity in home hemodialysis: a fresh perspective on time as a medium of Distributed Cognition. Cognition, Technology \& Work, 16(3), 337-348. https://doi.org/10.1007/s10111-013-0263-x

Rehmann, J. A., Mitman, R. D., \& Reynolds, M. C. (1995). A Handbook of Flight Simulation Fidelity Requirements for Human Factors Research. Atlantic City, NJ, USA: Federal Aviation Administration Technical Center.

Rescorla, M. (2017). The Computational Theory of Mind. In E. N. Zalta (Ed.), The Stanford Encyclopedia of Pbilosophy (Spring Ed.). Retrieved from https://plato.stanford.edu/archives/spr2017/entries/computational-mind/

Rogers, Y. (1997). A brief introduction to distributed cognition. United Kingdom, Europe. https://doi.org/10.1007/s00607

Rohrer, M. W. (2000). Seeing is Believing: The Importance of Visualization in Manufacturing Simulation. In J. A. Joines, R. R. Barton, K. Kang, \& P. a. Fishwick (Eds.), Proceedings of the 2000 Winter Simulation Conference (Vol. 2, pp. 12111216). Orlando, FL, USA. https://doi.org/10.1109/WSC.2000.899166

Rosen, K. R. (2008). The history of medical simulation. Journal of Critical Care, 23(2), 157-66. https://doi.org/10.1016/j.jcrc.2007.12.004

Rosen, M. A., Salas, E., Wilson, K. A., King, H. B., Salisbury, M. L., Augenstein, J. S., ... Birnbach, D. J. (2008). Measuring Team Performance in Simulation-Based Training: Adopting Best Practices for Healthcare. Simulation in Healthcare, 3(1), 33-41. https://doi.org/10.1097/SIH.0b013e3181626276

Rosen, M. A., \& Weaver, S. (2010). Tools for evaluating team performance in simulation-based training. Journal of Emergencies, Trauma, and Shock, 3(4), 353-359.

Rosenblueth, A., \& Wiener, N. (1945). The Role of Models in Science. Philosophy of Science, 12(4), 316-321. https://doi.org/10.1086/286874

Rothrock, L., \& Narayanan, S. (2011). Human-in-the-Loop Simulations. (L. Rothrock \& S. Narayanan, Eds.). London, UK: Springer-Verlag London Limited. https://doi.org/10.1007/978-0-85729-883-6

Rüter, A., Nilsson, H., \& Wikström, T. (2004). Prehospital sjukvårdsledning - en konceptutbildning med certifiering. Akuttjournalen: Tidsskrift for Avansert Akuttmedisin, 12(4), 148-149. 
Salas, E., Bowers, C. A., \& Rhodenizer, L. (1998). It Is Not How Much You Have but How You Use It: Toward a Rational Use of Simulation to Support Aviation Training. The International Journal of Aviation Psychology, 8(3), 197-208. https://doi.org/10.1207/s15327108ijap0803

Salas, E., Cooke, N. J., \& Rosen, M. A. (2008). On teams, teamwork, and team performance: discoveries and developments. Human Factors, 50(3), 540-7. https://doi.org/10.1518/001872008X288457.

Salas, E., Gregory, M., \& King, H. (2011). Team training can enhance patient safety--the data, the challenge ahead. Joint Commission Journal on Quality and Patient Safety, 37(8), 339-40.

Salas, E., Rosen, M. A., Held, J. D., \& Weissmuller, J. J. (2008). Performance Measurement in Simulation-Based Training: A Review and Best Practices. Simulation \& Gaming, 40(3), 328-376. https://doi.org/10.1177/1046878108326734

Sanders, E. B. (2002). From User-Centered to Participatory Design Approaches. In J. Frascara (Ed.), Design and the Social Sciences (p. 256). London, UK: Taylor \& Francis Books Limited. https://doi.org/10.1201/9780203301302.ch1

Sargent, R. G. (1996). Verification and Validation of Simulation Models. In Winter Simulation Conference (pp. 55-64). Coronado, CA: IEEE.

Sargent, R. G. (2012). Verification and validation of simulation models. Journal of Simulation, 7(1), 12-24. https://doi.org/10.1057/jos.2012.20

Sawyer, T. L., \& Deering, S. (2013). Adaptation of the US Army's After-Action Review for Simulation Debriefing in Healthcare. Simulation in Healthcare, 8(6), 388-397. https://doi.org/10.1097/SIH.0b013e31829ac85c

Scerbo, M. W., \& Dawson, S. (2007). High fidelity, high performance? Simulation in Healthcare, 2(4), 224-30. https://doi.org/10.1097/SIH.0b013e31815c25f1

Scerbo, M. W., Kennedy, R. A., Montano, M., Britt, R. C., Davis, S. S., \& Stefanidis, D. (2013). A Spatial Secondary Task for Measuring Laparoscopic Mental Workload. Proceedings of the Human Factors and Ergonomics Society Annual Meeting, 57(1), 728-732. https://doi.org/10.1177/1541931213571159

Schwarzer, R., \& Jerusalem, M. (1995). Generalized Self-Efficacy scale. In M. Johnston, J. Weinman, \& S. C. Wright (Eds.), Measures in bealth psychology: A user's porffolio. Causal and control beliefs (pp. 35-37). London, UK: GL Assessment. https://doi.org/10.1037/t00393-000

Searle, J. (1980). Minds, brains, and programs. Behavioral and Brain Sciences, 3(3), 417-424.

Sellberg, C., \& Lindblom, J. (2014). Comparing methods for workplace studies: A theoretical and empirical analysis. Cognition, Technology \& Work, 16(4), 467-486. https://doi.org/10.1007/s10111-014-0273-3

Seropian, M. A. (2003). General Concepts in Full Scale Simulation: Getting Started. Anesthesia \& Analgesia, 97(6), 1695-1705. https://doi.org/10.1213/01.ANE.0000090152.91261.D9

Shapere, D. (1998). Building on what we have learned: The relations between science and technology. Philosophy and Technology, $4(2), 105-120$.

Sharp, H., Rogers, Y., \& Preece, J. (2007). Interaction design: beyond buman-computer interaction (Vol. 11). Hoboken, NJ, USA: John Wiley And Sons Ltd. https://doi.org/10.1162/leon.2005.38.5.401

Sheridan, T. B., Wickens, C. D., Parasuraman, R., Sheridan, T. B., \& Wickens, C. D. (2008). Situation Awareness, Mental Workload, and Trust in Automation: Viable, Empirically Supported Cognitive Engineering Constructs. Human Factors, 2(2), 140-160. https://doi.org/10.1518/155534308X284417.

Singer, M., \& Hayes, R. (1989). Simulation Fidelity in Training System Design. (M. Singer \& R. Hayes, Eds.). New york, NY, USA: Springer-Verlag.

Siu, J., Maran, N., \& Paterson-Brown, S. (2016). Observation of behavioural markers of non-technical skills in the operating room and their relationship to intra-operative incidents. Surgeon, 14(3), 119-128. https://doi.org/10.1016/j.surge.2014.06.005

Smallman, H. S., \& John, M. S. (2005). Naive realism: misplaced faith in realistic displays. Ergonomics in Design: The Quarterly of Human Factors Applications, 13(3), 6-13. https://doi.org/10.1177/106480460501300303

Smart, P. R., \& Sycara, K. (2015). Situating Cognition within the Virtual World. In 6th International Conference on Applied Human Factors and Ergonomics (AHFE 2015) and the Affiliated Conferences, AHFE 2015 (pp. 3836-3843). Las Vegas, Nevada, USA: Elsevier B.V. https://doi.org/10.1016/j.promfg.2015.07.887

Sokolowski, J. A. (2010). Monte Carlo Simulation. In J. A. Sokolowski \& C. M. Banks (Eds.), Modeling and Simulation Fundementals: Theoretical Underpinnings and Practical Domains (pp. 131-145). Hoboken, NJ, USA: John Wiley \& Sons, Inc. 
Sokolowski, J. A., \& Banks, C. M. (2010). Modeling and Simulation Fundementals: Theoretical Underpinnings and Practical Domains. (J. A. Sokolowski \& C. M. Banks, Eds.). Hoboken, NJ, USA: John Wiley \& Sons, Inc.

Stoffregen, T. A., Bardy, B. G., Smart, L. J., \& Pagulayan, R. J. (2003). On the nature and evaluation of fidelity in virtual environments. In M. W. Haas \& L. J. Hettinger (Eds.), Psychological issues in the design and use of virtual environments (pp. 111-119). London, UK: Taylor \& Francis. https://doi.org/10.1201/9781410608888.ch6

Stratton, S. J., \& Tyler, R. D. (2006). Characteristics of Medical Surge Capacity Demand for Sudden-impact Disasters. Academic Emergency Medicine, 13(11), 1193-1197. https://doi.org/10.1197/j.aem.2006.05.008

Suchman, L. (1997). Centers of coordination: a case and some themes. In L. B. Resnick, R. Säljö, C. Pontecorvo, \& B. Burge (Eds.), Discourse, Tools and Reasoning - Essays on Situated Cognition (pp. 41-62). Berlin, Germany: Springer-Verlag. https://doi.org/10.1007/978-3-662-03362-3_3

Sundnes, K., \& Birnbaum, M. (2003). Health disaster management: Guidelines for evaluation and research in the Utstein style. Volume I. Conceptual framework of disasters. Prehospital and Disaster Medicine, 17(3), 1-177.

Thagard, P. (2014). Cognitive Science. In E. N. Zalta (Ed.), Stanford Encyclopedia of Philosophy (Fall Ed.). Retrieved from https://plato.stanford.edu/archives/fall2014/entries/cognitive-science

Toups, Z. O., \& Kerne, A. (2007). Implicit Coordination in Firefighting Practice. In Proceedings of the SIGCHI conference on Human factors in computing systems - CHI '07 (pp. 707-716). New york, NY, USA: ACM Press. https://doi.org/10.1145/1240624.1240734

Toups, Z. O., Kerne, A., Hamilton, W. A., \& Blevins, A. (2009). Emergent Team Coordination: From Fire Emergency Response Practice to a Non-Mimetic Simulation Game. In GROUP '09: Proceedings of the ACM 2009 International Conference on Supporting Group Work (pp. 341-350). Sanibel Island, FL, USA: ACM Press. https://doi.org/10.1145/1531674.1531725

Toups, Z. O., Kerne, A., Hamilton, W. A., \& Shahzad, N. (2011). Zero-Fidelity Simulation of Fire Emergency Response. In Proceedings of the 2011 annual conference on Human factors in computing systems - CHI '11 (pp. 1959-1968). New york, NY, USA: ACM Press. https://doi.org/10.1145/1978942.1979226

Tsang, P. S., \& Vidulich, M. A. (2002). Principles and Practice of Aviation Psychology. Human Factors in Transportation. London, UK: CRC Press.

Turing, A. (1937). On Computable Numbers, With an Application to the Entscheidungsproblem. Proceedings of the London Mathematical Society, 2(42), 230-265.

Van de Walle, B., Turoff, M., \& Hiltz, S. R. (2010). Information systems for emergency management. London, UK: Routledge.

Weaver, S. J., Dy, S. M., \& Rosen, M. A. (2014). Team-training in healthcare: a narrative synthesis of the literature. BMJ Quality \& Safety, 23(5), 359-72. https://doi.org/10.1136/bmjqs-2013-001848

Werth, J., \& Furniss, D. (2012). Medical Equipment Library design: Revealing issues and best practice using DiCoT. In Proceedings of the 2nd ACM SIGHIT International Health Informatics Symposium (pp. 583-592). New york, NY, USA: ACM Press. https://doi.org/10.1145/2110363.2110428

Winsberg, E. (2015). Computer Simulations in Science. In E. N. Zalta (Ed.), The Stanford Encyclopedia of Philosophy (Summer Ed., pp. 1-20). Retrieved from http://plato.stanford.edu/archives/sum2015/entries/simulations-science/

Witmer, B. G., \& Singer, M. J. (1998). Measuring Presence in Virtual Environments: A Presence Questionnaire. Presence: Teleoperators and Virtual Environments, 7(3), 225-240. https://doi.org/10.1162/105474698565686

Wolfe, J., \& Crookall, D. (1998). Developing a Scientific Knowledge of Simulation/Gaming. Simulation \& Gaming, 29(1), 719. https://doi.org/10.1177/1046878198291002

Wolfe, J., \& Jackson, R. (1989). An Investigation of the Need for Algorithmic Validity. Simulation \& Games, 20(3), $272-291$. https://doi.org/10.1177/104687818902000303

Wong, B. L. W., \& Blandford, A. (2001). Situation Awareness and its Implications for Human--Systems Interaction OzCHI 2001. In Proceedings of the Australian Conference on Computer-Human Interaction (pp. 181-186). Perth, Australia.

Woods, D. D. (1995). Symbolic Al Computer Simulations as Tools for Investigating the Dynamics of Joint Cognitive Systems. In P. C. Hoc, B. Cacciabue, \& E. Hollnagel (Eds.), Simulation of Cognition in Dynamic Environments (p. 75). Hillsdale, NJ, USA: Lawrence Erlbaum Associates.

Woolgar, S., \& Suchman, L. A. (1989). Plans and Situated Actions: The Problem of Human Machine Communication. Contemporary Sociology, 18(3), 414. https://doi.org/10.2307/2073874

Yurko, Y. Y., Scerbo, M. W., Prabhu, A. S., Acker, C. E., \& Stefanidis, D. (2010). Higher Mental Workload is Associated With Poorer Laparoscopic Performance as Measured by the NASA-TLX Tool. Simulation in Healthcare, 5(5), 267-271. https://doi.org/10.1097/SIH.0b013e3181e3f329 
Zhang, J., \& Norman, D. A. (1994). Representations in Distributed Cognitive Tasks. Cognitive Science, 18(1), 87-122. https://doi.org/10.1207/s15516709 $\operatorname{cog} 1801 \_3$ 


\section{Papers}

The papers associated with this thesis have been removed for copyright reasons. For more details about these see:

http://urn.kb.se/resolve?urn=urn:nbn:se:liu:diva-145726 
Linköping Studies in Science and Technology

No 14 Anders Haraldsson A Program Manipulation System Based on Partial Evaluation, 1977, ISBN 91-7372-144-1

No 17 Bengt Magnhagen: Probability Based Verification of Time Margins in Digital Designs, 1977, ISBN 91-7372-157-3

No 18 Mats Cedwall Semantisk analys av processbeskrivningar i naturligt språk, 1977, ISBN 91- 7372-168-9

No 22 Jaak Urmi: A Machine Independent LISP Compiler and its Implications for Ideal Hardware, 1978, ISBN 91-7372188-3

No 33 Tore Risch: Compilation of Multiple File Queries in a Meta-Database System, 1978, ISBN 91- 7372-232-4

No 51 Erland Jungert: Synthesizing Database Structures from a User Oriented Data Model, 1980, ISBN 917372-387-8

No 54 Sture Hägglund: Contributions to the Development of Methods and Tools for Interactive Design of Applications Software, 1980, ISBN 91-7372-404-1

No 55 Pär Emanuelson: Performance Enhancement in a Well-Structured Pattern Matcher through Partial Evaluation, 1980, ISBN 91-7372-403-3

No 58 Bengt Johnsson, Bertil Andersson: The Human-Computer Interface in Commercial Systems, 1981, ISBN 917372-414-9

No 69 H. Jan Komorowski: A Specification of an Abstract Prolog Machine and its Application to Partial Evaluation, 1981, ISBN 91-7372-479-3

No 71 René Reboh: Knowledge Engineering Techniques and Tools for Expert Systems, 1981, ISBN 91-7372-489-0

No 77 Östen Oskarsson: Mechanisms of Modifiability in large Software Systems, 1982, ISBN 91- 7372-527-7

No 94 Hans Lunell: Code Generator Writing Systems, 1983, ISBN 91-7372-652-4

No 97 Andrzej Lingas: Advances in Minimum Weight Triangulation, 1983, ISBN 91-7372-660-5

No 109 Peter Fritzson: Towards a Distributed Programming Environment based on Incremental Compilation, 1984, ISBN 91-7372-801-2

No 111 Erik Tengvald: The Design of Expert Planning Systems An Experimental Operations Planning System for Turning, 1984, ISBN 91-7372- 805-5

No 155 Christos Levcopoulos: Heuristics for Minimum Decompositions of Polygons, 1987, ISBN 91-7870-133-3
No 165 James W. Goodwin: A Theory and System for Non-Monotonic Reasoning, 1987, ISBN 91-7870-183-X

No 170 Zebo Peng: A Formal Methodology for Automated Synthesis of VLSI Systems, 1987, ISBN 91-7870-225-9

No 174 Johan Fagerström: A Paradigm and System for Design of Distributed Systems, 1988, ISBN 91-7870-301-8

No 192 Dimiter Driankov: Towards a Many Valued Logic of Quantified Belief, 1988, ISBN 91-7870-374-3

No 213 Lin Padgham: Non-Monotonic Inheritance for an Object Oriented Knowledge Base, 1989, ISBN 91-7870485-5

No 214 Tony Larsson: A Formal Hardware Description and Verification Method, 1989, ISBN 91-7870-517-7

No 221 Michael Reinfrank: Fundamentals and Logical Foundations of Truth Maintenance, 1989, ISBN 91-7870-5460

No 239 Jonas Löwgren: Knowledge-Based Design Support and Discourse Management in User Interface Management Systems, 1991, ISBN 917870-720-X

No 244 Henrik Eriksson: Meta-Tool Support for Knowledge Acquisition, 1991, ISBN 91-7870-746-3

No 252 Peter Eklund: An Epistemic Approach to Interactive Design in Multiple Inheritance Hierarchies, 1991, ISBN 91-7870-784-6

No 258 Patrick Doherty: NML3 - A NonMonotonic Formalism with Explicit Defaults, 1991, ISBN 91-7870-816-8

No 260 Nahid Shahmehri: Generalized Algorithmic Debugging, 1991, ISBN 91-7870-828-1

No 264 Nils Dahlbäck: Representation of Discourse-Cognitive and Computational Aspects, 1992, ISBN 91-7870-850-8

No 265 Ulf Nilsson: Abstract Interpretations and Abstract Machines Contributions to a Methodology for the Implementation of Logic Programs, 1992, ISBN 91-7870-858-3

No 270 Ralph Rönnquist: Theory and Practice of Tense-bound Object References, 1992, ISBN 91-7870-873-7

No 273 Björn Fjellborg: Pipeline Extraction for VLSI Data Path Synthesis, 1992, ISBN 91-7870-880-X

No 276 Staffan Bonnier: A Formal Basis for Horn Clause Logic with External 
Polymorphic Functions, 1992, ISBN 917870-896-6

No 277 Kristian Sandahl: Developing Knowledge Management Systems with an Active Expert Methodology, 1992, ISBN 91-7870-897-4

No 281 Christer Bäckström: Computational Complexity of Reasoning about Plans, 1992, ISBN 91-7870-979-2

No 292 Mats Wirén: Studies in Incremental Natural Language Analysis, 1992, ISBN 91-7871-027-8

No 297 Mariam Kamkar: Interprocedural Dynamic Slicing with Applications to Debugging and Testing, 1993, ISBN 917871-065-0

No 302 Tingting Zhang: A Study in Diagnosis Using Classification and Defaults, 1993, ISBN 91-7871-078-2

No 312 Arne Jönsson: Dialogue Management for Natural Language Interfaces - An Empirical Approach, 1993, ISBN 917871-110-X

No 338 Simin Nadjm-Tehrani: Reactive Systems in Physical Environments Compositional Modelling and Framework for Verification, 1994, ISBN 917871-237-8

No 371 Bengt Savén: Business Models for Decision Support and Learning A Study of Discrete-Event Manufacturing Simulation at Asea/ABB 1968-1993, 1995, ISBN 917871-494-X

No 375 Ulf Söderman: Conceptual Modelling of Mode Switching Physical Systems, 1995, ISBN 91-7871-516-4

No 383 Andreas Kågedal: Exploiting Groundness in Logic Programs, 1995, ISBN 91-7871-538-5

No 396 George Fodor: Ontological Control, Description, Identification and Recovery from Problematic Control Situations, 1995, ISBN 91-7871-603-9

No 413 Mikael Pettersson: Compiling Natural Semantics, 1995, ISBN 917871-641-1

No 414 Xinli Gu: RT Level Testability Improvement by Testability Analysis and Transformations, 1996, ISBN 917871-654-3

No 416 Hua Shu: Distributed Default Reasoning, 1996, ISBN 91-7871-665-9

No 429 Jaime Villegas: Simulation Supported Industrial Training from an Organisational Learning Perspective Development and Evaluation of the SSIT Method, 1996, ISBN 91-7871-7000
No 431 Peter Jonsson: Studies in Action Planning Algorithms and Complexity, 1996, ISBN 91-7871-704-3

No 437 Johan Boye: Directional Types in Logic Programming, 1996, ISBN 917871-725-6

No 439 Cecilia Sjöberg: Activities, Voices and Arenas Participatory Design in Practice, 1996, ISBN 91-7871-728-0

No 448 Patrick Lambrix: Part-Whole Reasoning in Description Logics, 1996, ISBN 91-7871-820-1

No 452 Kjell Orsborn: On Extensible and Object-Relational Database Technology for Finite Element Analysis Applications, 1996, ISBN 917871-827-9

No 459 Olof Johansson: Development Environments for Complex Product Models, 1996, ISBN 91-7871-855-4

No 461 Lena Strömbäck: User-Defined Constructions in Unification-Based Formalisms, 1997, ISBN 91-7871-857-0

No 462 Lars Degerstedt: Tabulation-based Logic Programming A Multi-Level View of Query Answering, 1996, ISBN 91-7871-858-9

No 475 Fredrik Nilsson: Strategi och ekonomisk styrning - En studie av hur ekonomiska styrsystem utformas och används efter företagsförvärv, 1997, ISBN 91-7871-914-3

No 480 Mikael Lindvall: An Empirical Study of Requirements-Driven Impact Analysis in Object-Oriented Software Evolution, 1997, ISBN 91-7871-927-5

No 485 Göran Forslund: Opinion-Based Systems The Cooperative Perspective on Knowledge-Based Decision Support, 1997, ISBN 91-7871-938-0

No 494 Martin Sköld: Active Database Management Systems for Monitoring and Control, 1997, ISBN 91-7219-002-7

No 495 Hans Olsén: Automatic Verification of Petri Nets in a CLP framework, 1997, ISBN 91-7219-011-6

No 498 Thomas Drakengren: Algorithms and Complexity for Temporal and Spatial Formalisms, 1997, ISBN 91-7219-019-1

No 502 Jakob Axelsson: Analysis and Synthesis of Heterogeneous Real-Time Systems, 1997, ISBN 91-7219-035-3

No 503 Johan Ringström: Compiler Generation for Data-Parallel Programming Languages from TwoLevel Semantics Specifications, 1997, ISBN 91-7219-045-0

No 512 Anna Moberg: Närhet och distans Studier av kommunikationsmönster $\mathrm{i}$ 
satellitkontor och flexibla kontor, 1997, ISBN 91-7219-119-8

No 520 Mikael Ronström: Design and Modelling of a Parallel Data Server for Telecom Applications, 1998, ISBN 917219-169-4

No 522 Niclas Ohlsson: Towards Effective Fault Prevention - An Empirical Study in Software Engineering, 1998, ISBN 91-7219-176-7

No526 Joachim Karlsson: A Systematic Approach for Prioritizing Software Requirements, 1998, ISBN 91-7219184-8

No530 Henrik Nilsson: Declarative Debugging for Lazy Functional Languages, 1998, ISBN 91-7219-197-X

No 555 Jonas Hallberg: Timing Issues in High-Level Synthesis, 1998, ISBN 917219-369-7

No 561 Ling Lin: Management of 1-D Sequence Data - From Discrete to Continuous, 1999, ISBN 91-7219-402-2

No 563 Eva L Ragnemalm: Student Modelling based on Collaborative Dialogue with a Learning Companion, 1999, ISBN 917219-412-X

No 567 Jörgen Lindström: Does Distance matter? On geographical dispersion in organisations, 1999, ISBN 91-7219-4391

No 582 Vanja Josifovski: Design, Implementation and Evaluation of a Distributed Mediator System for Data Integration, 1999, ISBN 91-7219-482-0

No 589 Rita Kovordányi: Modeling and Simulating Inhibitory Mechanisms in Mental Image Reinterpretation Towards Cooperative HumanComputer Creativity, 1999, ISBN 917219-506-1

No 592 Mikael Ericsson: Supporting the Use of Design Knowledge - An Assessment of Commenting Agents, 1999, ISBN 917219-532-0

No 593 Lars Karlsson: Actions, Interactions and Narratives, 1999, ISBN 91-7219534-7

No 594 C. G. Mikael Johansson: Social and Organizational Aspects of Requirements Engineering Methods A practice-oriented approach, 1999, ISBN 91-7219-541-X

No 595 Jörgen Hansson: Value-Driven MultiClass Overload Management in RealTime Database Systems, 1999, ISBN 91-7219-542-8

No 596 Niklas Hallberg: Incorporating User Values in the Design of Information
Systems and Services in the Public Sector A Methods Approach, 1999, ISBN 91-7219-543-6

No 597 Vivian Vimarlund: An Economic Perspective on the Analysis of Impacts of Information Technology From Case Studies in Health-Care towards General Models and Theories, 1999, ISBN 91-7219-544-4

No 598 Johan Jenvald: Methods and Tools in Computer-Supported Taskforce Training, 1999, ISBN 91-7219-547-9

No 607 Magnus Merkel: Understanding and enhancing translation by parallel text processing, 1999, ISBN 91-7219-614-9

No 611 Silvia Coradeschi: Anchoring symbols to sensory data, 1999, ISBN 91-7219-623-8

No 613 Man Lin: Analysis and Synthesis of Reactive Systems A Generic Layered Architecture Perspective, 1999, ISBN 91-7219-630-0

No 618 Jimmy Tjäder: Systemimplementering i praktiken En studie av logiker i fyra projekt, 1999, ISBN 91-7219-657-2

No 627 Vadim Engelson: Tools for Design, Interactive Simulation, and Visualization of Object-Oriented Models in Scientific Computing, 2000, ISBN 91-7219-709-9

No 637 Esa Falkenroth: Database Technology for Control and Simulation, 2000, ISBN 91-7219-766-8

No 639 Per-Arne Persson: Bringing Power and Knowledge Together Information Systems Design for Autonomy and Control in Command Work, 2000, ISBN 91-7219-796-X

No 660 Erik Larsson: An Integrated SystemLevel Design for Testability Methodology, 2000, ISBN 91-7219-8907

No 688 Marcus Bjäreland: Model-based Execution Monitoring, 2001, ISBN 917373-016-5

No 689 Joakim Gustafsson: Extending Temporal Action Logic, 2001, ISBN 917373-017-3

No 720 Carl-Johan Petri: Organizational Information Provision - Managing Mandatory and Discretionary Use of Information Technology, 2001, ISBN 91-7373-126-9

No 724 Paul Scerri: Designing Agents for Systems with Adjustable Autonomy, 2001, ISBN 91-7373-207-9 
No 725 Tim Heyer: Semantic Inspection of Software Artifacts From Theory to Practice, 2001, ISBN 91-7373-208-7

No 726 Pär Carlshamre: A Usability Perspective on Requirements Engineering - From Methodology to Product Development, 2001, ISBN 917373-212-5

No 732 Juha Takkinen: From Information Management to Task Management in Electronic Mail, 2002, ISBN 91-7373258-3

No 745 Johan Åberg: Live Help Systems An Approach to Intelligent Help for Web Information Systems, 2002, ISBN 917373-311-3

No 746 Rego Granlund: Monitoring Distributed Teamwork Training, 2002, ISBN 91-7373-312-1

No 757 Henrik André-Jönsson: Indexing Strategies for Time Series Data, 2002, ISBN 917373-346-6

No 747 Anneli Hagdahl: Development of ITsupported Interorganisational Collaboration - A Case Study in the Swedish Public Sector, 2002, ISBN 917373-314-8

No 749 Sofie Pilemalm: Information Technology for Non-Profit Organisations - $\quad$ Extended Participatory Design of an Information System for Trade Union Shop Stewards, 2002, ISBN 91-7373-318-0

No 765 Stefan Holmlid: Adapting users Towards a theory of use quality, 2002, ISBN 91-7373-397-0

No 771 Magnus Morin: Multimedia Representations of Distributed Tactical Operations, 2002, ISBN 917373-421-7

No 772 Pawel Pietrzak: A Type-Based Framework for Locating Errors in Constraint Logic Programs, 2002, ISBN 91-7373-422-5

No 758 Erik Berglund: Library Communication Among Programmers Worldwide, 2002, ISBN 91-7373-349-0

No 774 Choong-ho Yi: Modelling ObjectOriented Dynamic Systems Using a Logic-Based Framework, 2002, ISBN 91-7373-424-1

No 779 Mathias Broxvall: A Study in the Computational Complexity of Temporal Reasoning, 2002, ISBN 917373-440-3

No 793 Asmus Pandikow: A Generic Principle for Enabling Interoperability of Structured and Object-Oriented
Analysis and Design Tools, 2002, ISBN 91-7373-479-9

No 785 Lars Hult: Publika Informationstjänster En studie av den Internetbaserade encyklopedins bruksegenskaper, 2003, ISBN 91-7373461-6

No 800 Lars Taxén: A Framework for the Coordination of Complex Systems' Development, 2003, ISBN 91-7373-604$\mathrm{X}$

No 808 Klas Gäre: Tre perspektiv på förväntningar och förändringar $i$ samband med införande av informationssystem, 2003, ISBN 917373-618-X

No 821 Mikael Kindborg: Concurrent Comics - programming of social agents by children, 2003, ISBN 91-7373-651-1

No 823 Christina Ölvingson: On Development of Information Systems with GIS Functionality in Public Health Informatics A Requirements Engineering Approach, 2003, ISBN 917373-656-2

No 828 Tobias Ritzau: Memory Efficient Hard Real-Time Garbage Collection, 2003, ISBN 91-7373-666-X

No 833 Paul Pop: Analysis and Synthesis of Communication-Intensive

Heterogeneous Real-Time Systems, 2003, ISBN 91-7373-683-X

No 852 Johan Moe: Observing the Dynamic Behaviour of Large Distributed Systems to Improve Development and Testing - An Empirical Study in Software Engineering, 2003, ISBN 917373-779-8

No 867 Erik Herzog: An Approach to Systems Engineering Tool Data Representation and Exchange, 2004, ISBN 91-7373929-4

No 872 Aseel Berglund: Augmenting the Remote Control Studies in Complex Information Navigation for Digital TV, 2004, ISBN 91-7373-940-5

No 869 Jo Skåmedal: Telecommuting's Implications on Travel and Travel Patterns, 2004, ISBN 91-7373-935-9

No 870 Linda Askenäs: The Roles of IT Studies of Organising when Implementing and Using Enterprise Systems, 2004, ISBN 91-7373-936-7

No 874 Annika Flycht-Eriksson: Design and Use of Ontologies in InformationProviding Dialogue Systems, 2004, ISBN 91-7373-947-2 
No 873 Peter Bunus: Debugging Techniques for Equation-Based Languages, 2004, ISBN 91-7373-941-3

No 876 Jonas Mellin: Resource-Predictable and Efficient Monitoring of Events, 2004, ISBN 91-7373-956-1

No 883 Magnus Bång: Computing at the Speed of Paper Ubiquitous Computing Environments for Healthcare Professionals, 2004, ISBN 91-7373-971-5

No 882 Robert Eklund: Disfluency in Swedish human-human and human-machine travel booking dialogues, 2004, ISBN 91-7373-966-9

No 887 Anders Lindström: English and other Foreign Linguistic Elements in Spoken Swedish Studies of Productive Processes and their Modelling using Finite-State Tools, 2004, ISBN 91-7373981-2

No 889 Zhiping Wang: Capacity-Constrained Production-inventory systems Modelling and Analysis in both a traditional and an e-business context, 2004, ISBN 91-85295-08-6

No 893 Pernilla Qvarfordt: Eyes on Multimodal Interaction, 2004, ISBN 9185295-30-2

No 910 Magnus Kald: In the Borderland between Strategy and Management Control - Theoretical Framework and Empirical Evidence, 2004, ISBN 9185295-82-5

No 918 Jonas Lundberg: Shaping Electronic News Genre Perspectives on Interaction Design, 2004, ISBN 9185297-14-3

No 900 Mattias Arvola: Shades of use The dynamics of interaction design for sociable use, 2004, ISBN 91-85295-42-6

No 920 Luis Alejandro Cortés: Verification and Scheduling Techniques for RealTime Embedded Systems, 2004, ISBN 91-85297-21-6

No 929 Diana Szentivanyi: Performance Studies of Fault-Tolerant Middleware, 2005, ISBN 91-85297-58-5

No 933 Mikael Cäker: Management Accounting as Constructing and Opposing Customer Focus Three Case Studies on Management Accounting and Customer Relations, 2005, ISBN 91-85297-64-X

No 937 Jonas Kvarnström: TALplanner and Other Extensions to Temporal Action Logic, 2005, ISBN 91-85297-75-5

No 938 Bourhane Kadmiry: Fuzzy GainScheduled Visual Servoing for
Unmanned Helicopter, 2005, ISBN 9185297-76-3

No 945 Gert Jervan: Hybrid Built-In Self-Test and Test Generation Techniques for Digital Systems, 2005, ISBN 91-8529797-6

No 946 Anders Arpteg: Intelligent SemiStructured Information Extraction, 2005, ISBN 91-85297-98-4

No 947 Ola Angelsmark: Constructing Algorithms for Constraint Satisfaction and Related Problems - Methods and Applications, 2005, ISBN 91-85297-992

No 963 Calin Curescu: Utility-based Optimisation of Resource Allocation for Wireless Networks, 2005, ISBN 9185457-07-8

No 972 Björn Johansson: Joint Control in Dynamic Situations, 2005, ISBN 9185457-31-0

No 974 Dan Lawesson: An Approach to Diagnosability Analysis for Interacting Finite State Systems, 2005, ISBN 9185457-39-6

No 979 Claudiu Duma: Security and Trust Mechanisms for Groups in Distributed Services, 2005, ISBN 91-85457-54-X

No 983 Sorin Manolache: Analysis and Optimisation of Real-Time Systems with Stochastic Behaviour, 2005, ISBN 91-85457-60-4

No 986 Yuxiao Zhao: Standards-Based Application Integration for Businessto-Business Communications, 2005, ISBN 91-85457-66-3

No 1004 Patrik Haslum: Admissible Heuristics for Automated Planning, 2006, ISBN 91-85497-28-2

No 1005 Aleksandra Tešanovic: Developing Reusable and Reconfigurable RealTime Software using Aspects and Components, 2006, ISBN 91-85497-290

No 1008 David Dinka: Role, Identity and Work Extending the design and development agenda, 2006, ISBN 9185497-42-8

No 1009 Iakov Nakhimovski: Contributions to the Modeling and Simulation of Mechanical Systems with Detailed Contact Analysis, 2006, ISBN 9185497-43-X

No 1013 Wilhelm Dahllöf: Exact Algorithms for Exact Satisfiability Problems, 2006, ISBN 91-85523-97-6

No 1016 Levon Saldamli: PDEModelica - A High-Level Language for Modeling 
with Partial Differential Equations, 2006, ISBN 91-85523-84-4

No 1017 Daniel Karlsson: Verification of Component-based Embedded System Designs, 2006, ISBN 91-85523-79-8

No 1018 Ioan Chisalita: Communication and Networking Techniques for Traffic Safety Systems, 2006, ISBN 91-8552377-1

No 1019 Tarja Susi: The Puzzle of Social Activity - The Significance of Tools in Cognition and Cooperation, 2006, ISBN 91-85523-71-2

No 1021 Andrzej Bednarski: Integrated Optimal Code Generation for Digital Signal Processors, 2006, ISBN 9185523-69-0

No 1022 Peter Aronsson: Automatic Parallelization of Equation-Based Simulation Programs, 2006, ISBN 9185523-68-2

No 1030 Robert Nilsson: A Mutation-based Framework for Automated Testing of Timeliness, 2006, ISBN 91-85523-35-6

No 1034 Jon Edvardsson: Techniques for Automatic Generation of Tests from Programs and Specifications, 2006, ISBN 91-85523-31-3

No 1035 Vaida Jakoniene: Integration of Biological Data, 2006, ISBN 91-8552328-3

No 1045 Genevieve Gorrell: Generalized Hebbian Algorithms for Dimensionality Reduction in Natural Language Processing, 2006, ISBN 9185643-88-2

No 1051 Yu-Hsing Huang: Having a New Pair of Glasses - Applying Systemic Accident Models on Road Safety, 2006, ISBN 91-85643-64-5

No 1054 Åsa Hedenskog: Perceive those things which cannot be seen - A Cognitive Systems Engineering perspective on requirements management, 2006, ISBN 91-85643-57-2

No 1061 Cécile Åberg: An Evaluation Platform for Semantic Web Technology, 2007, ISBN 91-85643-31-9

No 1073 Mats Grindal: Handling Combinatorial Explosion in Software Testing, 2007, ISBN 978-91-85715-74-9

No 1075 Almut Herzog: Usable Security Policies for Runtime Environments, 2007, ISBN 978-91-85715-65-7

No 1079 Magnus Wahlström: Algorithms, measures, and upper bounds for Satisfiability and related problems, 2007, ISBN 978-91-85715-55-8
No 1083 Jesper Andersson: Dynamic Software Architectures, 2007, ISBN 978-9185715-46-6

No 1086 Ulf Johansson: Obtaining Accurate and Compre-hensible Data Mining Models - An Evolutionary Approach, 2007, ISBN 978-91-85715-34-3

No 1089 Traian Pop: Analysis and Optimisation of Distributed Embedded Systems with Heterogeneous Scheduling Policies, 2007, ISBN 978-91-85715-27-5

No 1091 Gustav Nordh: Complexity Dichotomies for CSP-related Problems, 2007, ISBN 978-91-85715-206

No 1106 Per Ola Kristensson: Discrete and Continuous Shape Writing for Text Entry and Control, 2007, ISBN 978-9185831-77-7

No 1110 He Tan: Aligning Biomedical Ontologies, 2007, ISBN 978-91-8583156-2

No 1112 Jessica Lindblom: Minding the body Interacting socially through embodied action, 2007, ISBN 978-91-85831-48-7

No 1113 Pontus Wärnestål: Dialogue Behavior Management in Conversational Recommender Systems, 2007, ISBN 978-91-85831-47-0

No 1120 Thomas Gustafsson: Management of Real-Time Data Consistency and Transient Overloads in Embedded Systems, 2007, ISBN 978-91-85831-333

No 1127 Alexandru Andrei: Energy Efficient and Predictable Design of Real-time Embedded Systems, 2007, ISBN 97891-85831-06-7

No 1139 Per Wikberg: Eliciting Knowledge from Experts in Modeling of Complex Systems Managing Variation and Interactions, 2007, ISBN 978-91-8589566-3

No 1143 Mehdi Amirijoo: QoS Control of RealTime Data Services under Uncertain Workload, 2007, ISBN 978-91-8589549-6

No 1150 Sanny Syberfeldt: Optimistic Replication with Forward Conflict Resolution in Distributed Real-Time Databases, 2007, ISBN 978-91-8589527-4

No 1155 Beatrice Alenljung: Envisioning a Future Decision Support System for Requirements Engineering - A Holistic and Human-centred Perspective, 2008, ISBN 978-91-85895-11-3 
No 1156 Artur Wilk: Types for XML with Application to Xcerpt, 2008, ISBN 97891-85895-08-3

No 1183 Adrian Pop: Integrated Model-Driven Development Environments for Equation-Based Object-Oriented Languages, 2008, ISBN 978-91-7393895-2

No 1185 Jörgen Skågeby: Gifting Technologies - Ethnographic Studies of End-users and Social Media Sharing, 2008, ISBN 978-91-7393-892-1

No 1187 Imad-Eldin Ali Abugessaisa: Analytical tools and informationsharing methods supporting road safety organizations, 2008, ISBN 97891-7393-887-7

No 1204 H. Joe Steinhauer: A Representation Scheme for Description and Reconstruction of Object Configurations Based on Qualitative Relations, 2008, ISBN 978-91-7393-8235

No 1222 Anders Larsson: Test Optimization for Core-based System-on-Chip, 2008, ISBN 978-91-7393-768-9

No 1238 Andreas Borg: Processes and Models for Capacity Requirements in Telecommunication Systems, 2009, ISBN 978-91-7393-700-9

No 1240 Fredrik Heintz: DyKnow A StreamBased Know-ledge Processing Middleware Framework, 2009, ISBN 978-91-7393-696-5

No 1241 Birgitta Lindström: Testability of Dynamic Real-Time Systems, 2009, ISBN 978-91-7393-695-8

No 1244 Eva Blomqvist: Semi-automatic Ontology Construction based on Patterns, 2009, ISBN 978-91-7393-6835

No 1249 Rogier Woltjer: Functional Modeling of Constraint Management in Aviation Safety and Command and Control, 2009, ISBN 978-91-7393-659-0

No 1260 Gianpaolo Conte: Vision-Based Localization and Guidance for Unmanned Aerial Vehicles, 2009, ISBN 978-91-7393-603-3

No 1262 AnnMarie Ericsson: Enabling Tool Support for Formal Analysis of ECA Rules, 2009, ISBN 978-91-7393-598-2

No 1266 Jiri Trnka: Exploring Tactical Command and Control A RolePlaying Simulation Approach, 2009, ISBN 978-91-7393-571-5

No 1268 Bahlol Rahimi: Supporting Collaborative Work through ICT How End-users Think of and Adopt
Integrated Health Information Systems, 2009, ISBN 978-91-7393-5500

No 1274 Fredrik Kuivinen: Algorithms and Hardness Results for Some Valued CSPs, 2009, ISBN 978-91-7393-525-8

No 1281 Gunnar Mathiason: Virtual Full Replication for Scalable Distributed Real-Time Databases, 2009, ISBN 97891-7393-503-6

No 1290 Viacheslav Izosimov: Scheduling and Optimization of Fault-Tolerant Distributed Embedded Systems, 2009, ISBN 978-91-7393-482-4

No 1294 Johan Thapper: Aspects of a Constraint Optimisation Problem, 2010, ISBN 978-91-7393-464-0

No 1306 Susanna Nilsson: Augmentation in the Wild User Centered Development and Evaluation of Augmented Reality Applications, 2010, ISBN 978-91-7393416-9

No 1313 Christer Thörn: On the Quality of Feature Models, 2010, ISBN 978-917393-394-0

No 1321 Zhiyuan He: Temperature Aware and Defect-Probability Driven Test Scheduling for System-on-Chip, 2010, ISBN 978-91-7393-378-0

No 1333 David Broman: Meta-Languages and Semantics for Equation-Based Modeling and Simulation, 2010, ISBN 978-91-7393-335-3

No 1337 Alexander Siemers: Contributions to Modelling and Visualisation of Multibody Systems Simulations with Detailed Contact Analysis, 2010, ISBN 978-91-7393-317-9

No 1354 Mikael Asplund: Disconnected Discoveries Availability Studies in Partitioned Networks, 2010, ISBN 97891-7393-278-3

No 1359 Jana Rambusch Mind Games Extended Understanding Gameplay as Situated Activity, 2010, ISBN 97891-7393-252-3

No 1373 Sonia Sangari Head Movement Correlates to Focus Assignment in Swedish, 2011, ISBN 978-91-7393-1540

No 1374 Jan-Erik Källhammer Using False Alarms when Developing Automotive Active Safety Systems, 2011, ISBN 97891-7393-153-3

No 1375 Mattias Eriksson Integrated Code Generation, 2011, ISBN 978-91-7393147-2

No 1381 Ola Leifler Affordances and Constraints of Intelligent Decision 
Support for Military Command and Control - Three Case Studies of Support Systems, 2011, ISBN 978-917393-133-5

No 1386 Soheil Samii Quality-Driven Synthesis and Optimization of Embedded Control Systems, 2011, ISBN 978-91-7393-102-1

No 1419 Erik Kuiper Geographic Routing in Intermittently-connected Mobile Ad Hoc Networks Algorithms and Performance Models, 2012, ISBN 97891-7519-981-8

No 1451 Sara Stymne Text Harmonization Strategies for Phrase-Based Statistical Machine Translation, 2012, ISBN 97891-7519-887-3

No 1455 Alberto Montebelli Modeling the Role of Energy Management in Embodied Cognition, 2012, ISBN 97891-7519-882-8

No 1465 Mohammad Saifullah BiologicallyBased Interactive Neural Network Models for Visual Attention and Object Recognition, 2012, ISBN 978-917519-838-5

No 1490 Tomas Bengtsson Testing and Logic Optimization Techniques for Systems on Chip, 2012, ISBN 978-91-7519-742-5

No 1481 David Byers Improving Software Security by Preventing Known Vulnerabilities, 2012, ISBN 978-917519-784-5

No1496 Tommy Färnqvist Exploiting Structure in CSP-related Problems, 2013, ISBN 978-91-7519-711-1

No 1503 John Wilander Contributions to Specification, Implementation, and Execution of Secure Software, 2013, ISBN 978-91-7519-681-7

No 1506 Magnus Ingmarsson Creating and Enabling the Useful Service Discovery Experience, 2013, ISBN 978-91-7519662-6

No 1547 Wladimir Schamai Model-Based Verification of Dynamic System Behavior against Requirements Method, Language, and Tool, 2013, ISBN 978-91-7519-505-6

No 1551 Henrik Svensson Simulations, 2013, ISBN 978-91-7519-491-2

No 1559 Sergiu Rafiliu Stability of Adaptive Distributed Real-Time Systems with Dynamic Resource Management, 2013, ISBN 978-91-7519-471-4

No 1581 Usman Dastgeer Performance-aware Component Composition for GPUbased Systems, 2014, ISBN 978-917519-383-0
No 1602 Cai Li Reinforcement Learning of Locomotion based on Central Pattern Generators, 2014, ISBN 978-91-7519313-7

No 1652 Roland Samlaus An Integrated Development Environment with Enhanced Domain-Specific Interactive Model Validation, 2015, ISBN 978-917519-090-7

No 1663 Hannes Uppman On Some Combinatorial Optimization Problems Algorithms and Complexity, 2015, ISBN 978-91-7519072-3

No 1664 Martin Sjölund Tools and Methods for Analysis, Debugging, and Performance Improvement of Equation-Based Models, 2015, ISBN 978-91-7519-071-6

No 1666 Kristian Stavåker Contributions to Simulation of Modelica Models on Data-Parallel Multi-Core Architectures, 2015, ISBN 978-91-7519068-6

No 1680 Adrian Lifa Hardware/Software Codesign of Embedded Systems with Reconfigurable and Heterogeneous Platforms, 2015, ISBN 978-91-7519040-2

No 1685 Bogdan Tanasa Timing Analysis of Distributed Embedded Systems with Stochastic Workload and Reliability Constraints, 2015, ISBN 978-91-7519022-8

No 1691 Håkan Warnquist Troubleshooting Trucks - Automated Planning and Diagnosis, 2015, ISBN 978-91-7685993-3

No 1702 Nima Aghaee Thermal Issues in Testing of Advanced Systems on Chip, 2015, ISBN 978-91-7685-949-0

No 1715 Maria Vasilevskaya Security in Embedded Systems A Model-Based Approach with Risk Metrics, 2015, ISBN 978-91-7685-917-9

No 1729 Ke Jiang Security-Driven Design of Real-Time Embedded System, 2016, ISBN 978-91-7685-884-4

No 1733 Victor Lagerkvist Strong Partial Clones and the Complexity of Constraint Satisfaction Problems Limitations and Applications, 2016, ISBN 978-91-7685-856-1

No 1734 Chandan Roy An Informed System Development Approach to Tropical Cyclone Track and Intensity Forecasting, 2016, ISBN 978-91-7685854-7

No 1746 Amir Aminifar Analysis, Design, and Optimization of Embedded Control 
Systems, 2016, ISBN 978-91-7685-8264

No 1747 Ekhiotz Vergara Energy Modelling and Fairness for Efficient Mobile Communication, 2016, ISBN 978-917685-822-6

No 1748 Dag Sonntag Chain Graphs Interpretations, Expressiveness and Learning Algorithms, 2016, ISBN 97891-7685-818-9

No 1768 Anna Vapen Web Authentication using Third-Parties in Untrusted Environments, 2016, ISBN 978-917685-753-3

No 1778 Magnus Jandinger On a Need to Know Basis A Conceptual and Methodological Framework for Modelling and Analysis of Information Demand in an Enterprise Context, 2016, ISBN 978-91-7685-713-7

No 1798 Rahul Hiran Collaborative Network Security Targeting Wide-area Routing and Edge-network Attacks, 2016, ISBN 978-91-7685-662-8

No 1813 Nicolas Melot Algorithms and Framework for Energy Efficient Parallel Stream Computing on ManyCore Architectures, 2016, ISBN 978-917685-623-9

No 1823 Amy Rankin Making Sense of Adaptations Resilience in High-Risk Work, 2017, ISBN 978-91-7685-596-6

No 1831 Lisa Malmberg Building Design Capability in the Public Sector Expanding the Horizons of Development, 2017, ISBN 978-91-7685585-0

No 1851 Marcus Bendtsen Gated Bayesian Networks, 2017, ISBN 978-91-7685525-6

No 1852 Zlatan Dragisic Completion of Ontologies and Ontology Networks, 2017, ISBN 978-91-7685-522-5

No 1854 Meysam Aghighi Computational Complexity of some Optimization Problems in Planning, 2017, ISBN 97891-7685-519-5

No 1863 Simon Ståhlberg Methods for Detecting Unsolvable Planning Instances using Variable Projection, 2017, ISBN 978-91-7685-498-3

No 1879 Karl Hammar Content Ontology Design Patterns Qualities, Methods, and Tools, 2017, ISBN 978-91-7685454-9

No 1887 Ivan Ukhov System-Level Analysis and Design under Uncertainty, 2017, ISBN 978-91-7685-426-6
No 1891 Valentina Ivanova Fostering User Involvement in Ontology Alignment and Alignment Evaluation, 2017, ISBN 978-91-7685-403-7

No 1902 Vengatanathan Krishnamoorthi Efficient HTTP-based Adaptive Streaming of Linear and Interactive Videos, 2018, ISBN 978-91-7685-371-9

No 1903 Lu Li Programming Abstractions and Optimization Techniques for GPUbased Heterogeneous Systems, 2018, ISBN 978-91-7685-370-2

No 1913 Jonas Rybing Studying Simulations with Distributed Cognition, 2018, ISBN 978-91-7685-348-1

\section{Linköping Studies in Arts and Science}

No 504 Ing-Marie Jonsson: Social and Emotional Characteristics of Speechbased In-Vehicle Information Systems Impact on Attitude and Driving Behaviour, 2009, ISBN 978-91-7393478-7

No 586 Fabian Segelström: Stakeholder Engagement for Service Design How service designers identify and communicate insights, 2013, ISBN 97891-7519-554-4

No 618 Johan Blomkvist: Representing Future Situations of Service Prototyping in Service Design, 2014, ISBN 978-91-7519-343-4

No 620 Marcus Mast: Human-Robot Interaction for Semi-Autonomous Assistive Robots, 2014, ISBN 978-917519-319-9

No 677 Peter Berggren: Assessing Shared Strategic Understanding, 2016, ISBN 978-91-7685-786-1

No 695 Mattias Forsblad: Distributed cognition in home environments The prospective memory and cognitive practices of older adults, 2016, ISBN 978-91-7685-686-4

Linköping Studies in Statistics

No 9 Davood Shahsavani: Computer Experiments Designed to Explore and Approximate Complex Deterministic Models, 2008, ISBN 978-91-7393-976-8

No 10 Karl Wahlin: Roadmap for Trend Detection and Assessment of Data Quality, 2008, ISBN 978-91-7393-792-4

No 11 Oleg Sysoev: Monotonic regression for large multivariate datasets, 2010, ISBN 978-91-7393-412-1

No 13 Agné Burauskaite-Harju: Characterizing Temporal Change and Inter-Site Correlations in Daily and 
Sub-daily Precipitation Extremes, 2011, ISBN 978-91-7393-110-6

Linköping Studies in Information Science

No 1

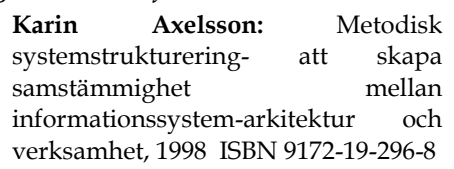

No 2 Stefan Cronholm: Metodverktyg och användbarhet - en studie av datorstödd metodbaserad systemutveckling, 1998, ISBN 9172-19299-2

No 3 Anders Avdic: Användare och utvecklare - om anveckling med kalkylprogram, 1999 ISBN 91-7219606-8

No 4 Owen Eriksson:

Kommunikationskvalitet hos informationssystem och affärsprocesser, 2000, ISBN 91-7219-811-7

No 5 Mikael Lind: Från system till process kriterier för processbestämning vid verksamhetsanalys, 2001, ISBN 917373-067-X

No 6 Ulf Melin: Koordination och informationssystem i företag och nätverk, 2002, ISBN 91-7373-278-8

No 7 Pär J. Ågerfalk: Information Systems Actability - Understanding Information Technology as a Tool for Business Action and Communication, 2003, ISBN 91-7373-628-7

No $8 \quad$ Ulf Seigerroth: Att förstå och förändra system-utvecklingsverksamheter - en taxonomi för metautveckling, 2003, ISBN 91-7373-736-4

No 9 Karin Hedström: Spår av datoriseringens värden -

Effekter av IT i äldreomsorg, 2004, ISBN 91-7373-963-4

No 10 Ewa Braf: Knowledge Demanded for Action - Studies on Knowledge Mediation in Organisations, 2004, ISBN 91-85295-47-7

No 11 Fredrik Karlsson: Method Configuration method and computerized tool support, 2005, ISBN 91-85297-48-8

No 12 Malin Nordström: Styrbar systemförvaltning - Att organisera systemförvaltningsverksamhet med hjälp av effektiva förvaltningsobjekt, 2005, ISBN 91-85297-60-7

No 13 Stefan Holgersson: Yrke POLIS Yrkeskunskap, motivation, IT-system och andra förutsättningar för polisarbete, 2005, ISBN 91-85299-43-X
No 14 Benneth Christiansson, MarieTherese Christiansson: Mötet mellan process och komponent - mot ett ramverk för en verksamhetsnära kravspecifikation vid anskaffning av komponent-baserade

informationssystem, 2006, ISBN 9185643-22-X 